\title{
ANALYSIS OF A CARBON FIBER REINFORCED POLYMER IMPACT ATTENUATOR FOR A FORMULA SAE VEHICLE USING FINITE ELEMENT ANALYSIS
}

\author{
A Thesis \\ presented to \\ the Faculty of California Polytechnic State University, \\ San Luis Obispo
}

\author{
In Partial Fulfillment \\ of the Requirements for the Degree \\ Master of Science in Mechanical Engineering
}

by

John Thomas Rappolt

June 2015 
John Thomas Rappolt

\section{ALL RIGHTS RESERVED}




\section{COMMITTEE MEMBERSHIP}

TITLE:

AUTHOR:

DATE SUBMITTED:

COMMITTEE CHAIR:

COMMITTEE MEMBER:

COMMITTEE MEMBER:
Analysis of a Carbon Fiber Reinforced Polymer Impact Attenuator for a Formula SAE Vehicle Using Finite Element Analysis

John Thomas Rappolt

June 2015

Dr. Joseph Mello, Ph.D.

Professor of Mechanical Engineering

Dr. Peter Schuster, Ph.D.

Professor of Mechanical Engineering

Dr. Eric Kasper, Ph.D.

Professor of Civil Engineering 


\begin{abstract}
Analysis of a Carbon Fiber Reinforced Polymer Impact Attenuator for a Formula SAE Vehicle Using Finite Element Analysis

John Thomas Rappolt

The Hashin failure criteria and damage evolution model for laminated fiber reinforced polymers are explored. A series of tensile coupon finite element analyses are run to characterize the variables in the physical model as well as modeling techniques for using an explicit dynamic solver for a quasi-static problem. An attempt to validate the model on an axial tube crush is presented. It was found that fiber buckling was not occurring at the impactor-tube interface. Results and speculation as to why the failure initiation is incorrect are discussed. Lessons learned from the tube crush are applied successfully to the quasi-static Formula SAE nosecone crush test. The model is validated by experimental data and the impact metrics between the test and model are within $5 \%$. Future work and possible optimization techniques are discussed.
\end{abstract}

Keywords: FSAE, FEA, CFRP, Abaqus, composite, progressive damage 


\section{ACKNOWLEDGMENTS}

Firstly, I would like thank my committee for introducing me to composites, finite element modeling, and structural analysis. Dr. Schuster and Dr. Kasper both introduced me to the theory of finite element analysis and I cannot thank them enough for doing so. I have become very fascinated with the tool and am looking forward to understanding it more deeply as my career progresses. Dr. Mello was brave enough to advise me on both my senior project and this Master's thesis and I am forever grateful. He introduced me to the world of composites, everything from design and manufacture to complex failure analysis. Because of these three gentlemen, I have found my passion in engineering and I cannot say thank you enough.

Secondly, I would like to thank Mr. Mike Freestone for his donation of the tubes for the crush experiment for this thesis. I would also like to thank him for providing me with the opportunity to work on spacecraft as a structural engineer, first as an intern and currently as a full time employee at SSL. In my short time at SSL, it has been nothing but a "dream come true" getting to work with these incredible machines and engineers and I am looking forward to the years to come.

Thirdly, I would like to thank the Cal Poly Formula SAE team for providing an incredible college experience that lead to the inspiration of this thesis. Specifically, I would like to thank the members of the 2013 chassis team, Nick Henderson, Matt Hagan and John Waldrop, and to Matt Roberts who provided the groundwork for this thesis. They and the rest of my teammates have all been a constant source of inspiration in their engineering knowledge, work ethic, and friendship. I will never forget our time together on the team.

Last and certainly not least, I would like to thank my family for their constant support over the course of my education. To my brother Andrew who pushes me to be the best I can possibly be (sibling rivalry can be a good thing). To my parents, Tom and Beth, have unconditionally supported me technically, financially, and emotionally for as long as I can remember. Without them, I would not be where I am today and I am truly and forever grateful for their love and support.

-- JTR 
LIST OF TABLES......................................................viii

LIST OF FIGURES......................................................

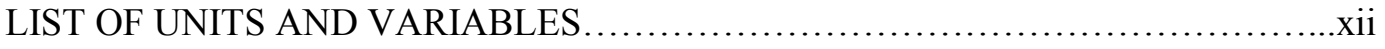

CHAPTER

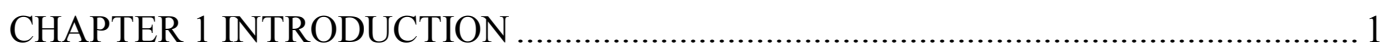

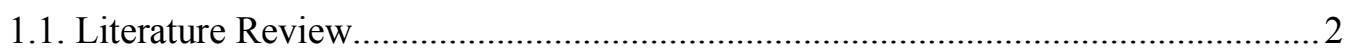

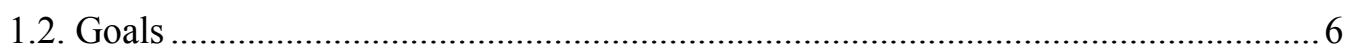

CHAPTER 2 OVERVIEW OF THE MODELING PROCESS ...................................... 7

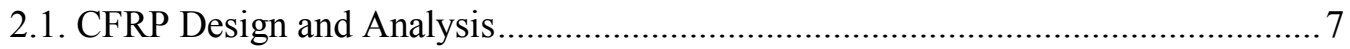

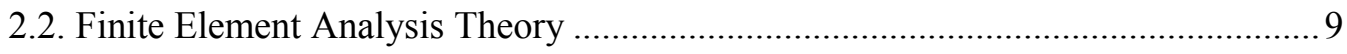

2.2.1. Implicit versus Explicit Integration in Transient Dynamic FEA .............. 10

2.3. Modeling Damage of Fiber Reinforced Composites in FEA ................................ 11

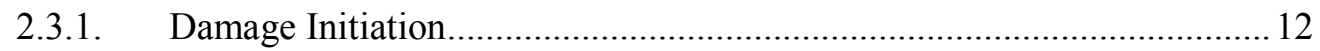

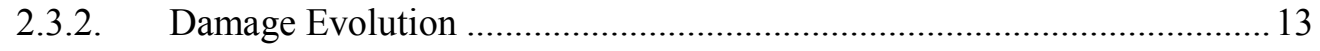

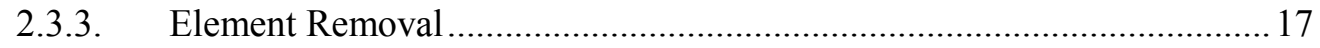

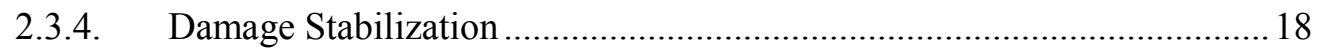

2.4. Quasi-Static Considerations with Explicit Dynamic FEA .................................... 19

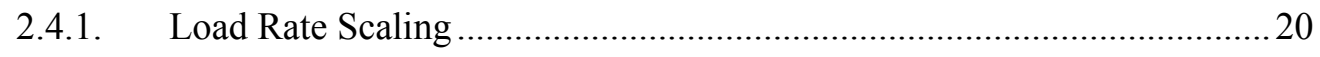

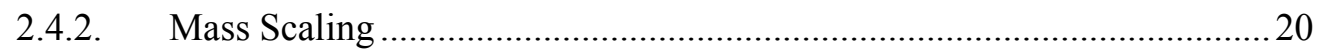

2.4.3. Smooth Step Load Application ........................................................... 21

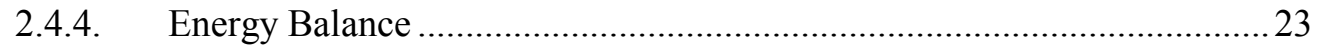

CHAPTER 3 PRACTICAL EVALUATION OF THE HASHIN DAMAGE

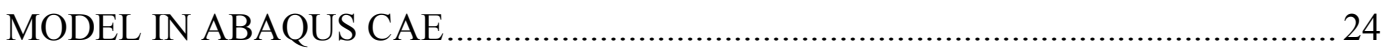

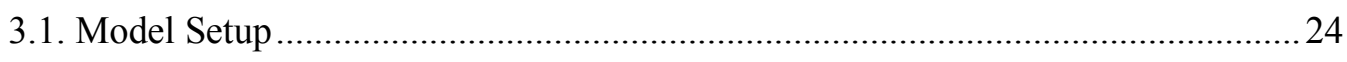

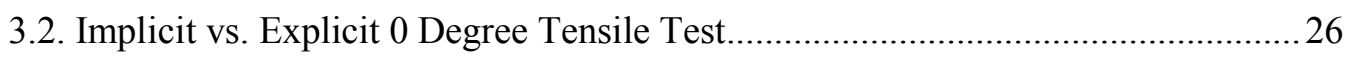

3.3. Mass Scaling and Smooth Step Amplitude Curve Application ............................29

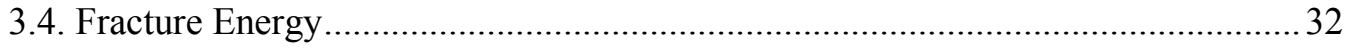

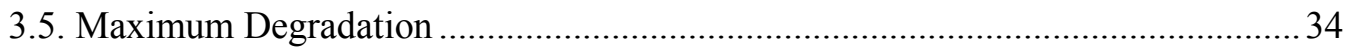

CHAPTER 4 AXIAL TUBE CRUSH TESTING AND FEA …..................................... 37

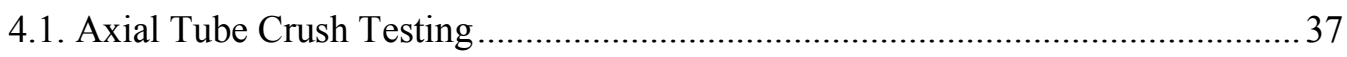

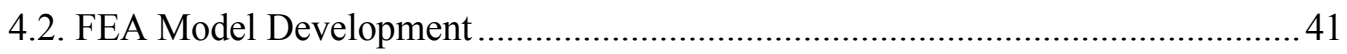

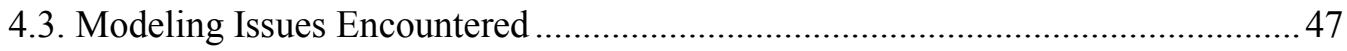

4.4. Finite Element Model Results and Discussion ................................................... 48 
CHAPTER 5 NOSECONE QUASI-STATIC CRUSH MODEL .................................... 55

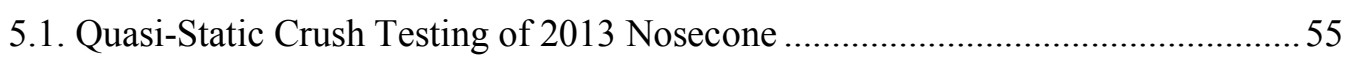

5.2. Finite Element Model Development ...................................................................... 59

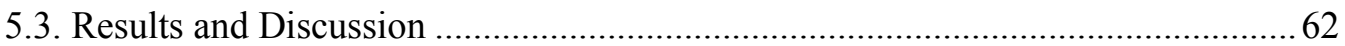

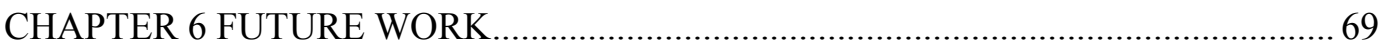

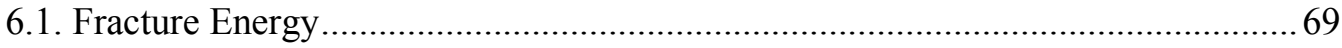

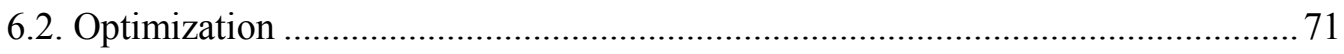

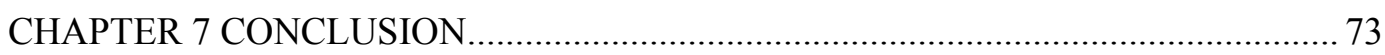

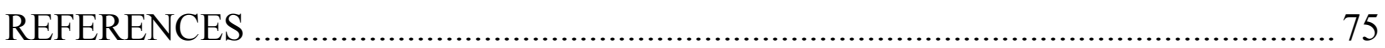

APPENDICES

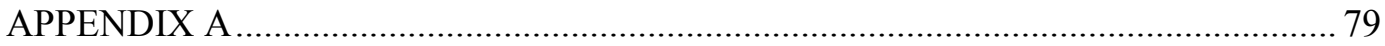

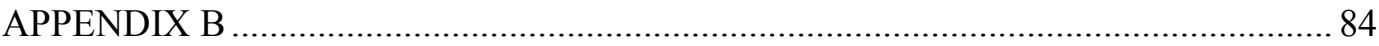

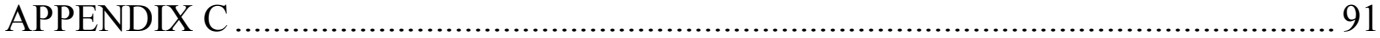




\section{LIST OF TABLES}

Table

Page

Table 3-1 - Material properties for CFRP used in tensile FEA experiments. ...................25

Table 3-2 - Dimensions of tensile specimen used in FEA experiments..........................26

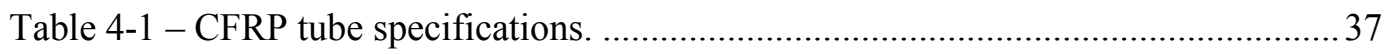

Table 4-2 - Crush performance characteristics of CFRP tubes. ..................................... 41

Table 4-3 - Material properties for M55J/RS-3C unidirectional tape CFRP. .................. 43

Table 4-4 - Material properties used of T300/RS-3C cloth CFRP................................. 44

Table 4-5 - Layup definitions for various sections of the tube ....................................... 45

Table 5-1 - Layup schedule for the 2013 Cal Poly FSAE Nosecone. ..............................56

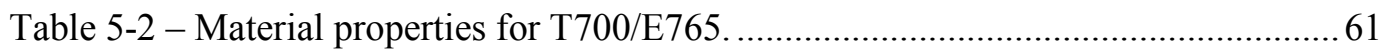

Table 5-3 - Impact metrics for Finite Element model and test nosecone........................67

Table 6-1 - Example Taguchi L4 Orthogonal Array..................................................... 72 


\section{LIST OF FIGURES}

Figure

Page

Figure 1-1 - Energry absorption by metallic impact structure [2] .............................. 3

Figure 1-2 - Energy absorption of composite impact structure showing catastrophic and progressive failure [2].

Figure 2-1 - Equivalent stress versus equivalent displacement. Note that the figure above shows the material completely fails at twice the displacement of damage initiation. This is not always true for all materials.

Figure 2-2 - Evolution of the damage variable with failure displacement $\delta e q f=2 \delta e q 0$ (a) and $20 \delta e q 0(\mathrm{~b})$.

Figure 2-3 - Definition of failure energy used to determine $\delta e q f$. A typical fiber reinforced polymer composite is shown in (a). The assumed CFRP stress-displacement curve is shown in (b).

Figure 2-4 - Example of deformed model with status variable not utilized in the visualizer.

Figure 2-5 - Smooth step amplitude curves for (a) one defined amplitude point and (b) multiple defined amplitude points [17]

Figure 2-6 - Impact displacement curves with various single amplitude point Smooth Step curves applied to impact velocity boundary conditions [17].

Figure 2-7 - Ideal quasi-static energy curve, with external work equal to internal energy and kinetic energy equal to zero.

Figure 3-1 - Quarter symmetry tensile coupon with boundary conditions shown [18]

Figure 3-2 - Abaqus/Standard results for CFRP tensile specimen using various mesh sizes.

Figure 3-3 - Abaqus/Explicit results for CFRP tensile specimen using various mesh sizes.

Figure 3-4 - Mesh size dependency results for Hashin damage model using a $\left[0_{6}\right]$ tensile speciemen.

Figure 3-5 - Load versus displacement plot for 0 degree tensile specimen using various mass scaling factors.

Figure 3-6 - Time to solution for various mass scaling factors.

Figure 3-7 - Kinetic energy versus internal energy for 0 degree tensile specimen using a mass scaling factor of $10^{10}$.

Figure 3-8 - Load versus displacement for 0 degree tensile specimen utilizing different smooth step amplitude times.

Figure 3-9 - Load versus displacement for 0 degree tensile specimen utilizing different fiber fracture energies. 
Figure 3-10 - Force versus deflection curve for 0 degree tensile specimen with varying maximum degradation values.

Figure 3-11 - Detailed view of damaged portion of load versus displacement response of 0 degree tensile specimen.

Figure 3-12 - Deformed shaped of 0 degree tensile specimen for $D_{\max }$ value of

(a) 1 , (b) 0.8 , (c) 0.6 , (d) 0.4, and (e) 0.2 .

Figure 4-1 - CAD drawing of tube crush test fixture.

Figure 4-2 - Post crush of specimen (a) 1A, (b) 2A, and (c) 3A. (d) Typical debris after a crush test.

Figure 4-3 - Load versus displacement data from tube axial crush tests.

Figure 4-4 - Specific crushing stress versus displacement for axial crush test of CFRP tubes.

Figure 4-5 - Trigger portion of two layer tube FEM.

Figure 4-6 - Overview of two layer tube FEM with plate instance removed. Turquoise section represents fully integrated elements; grey section represents reduced integration elements.

Figure 4-7 - Internal and kinetic energy for the entire tube crush model through the analyzed time period.

Figure 4-8 - Force versus displacement for test subject and FE analysis.

Figure 4-9 - Deformed tube shape with displacement magnitude contour at the initiation of crushing load.

Figure 4-10 - Tube crush with trigger $D_{\max }=0.5,0.2$ in post trigger set to $D_{\max }$ $=0.85$. Run terminated early due to undesirable results.

Figure 4-11 - Load vs. displacement for run with bulk viscosity set to 0.5 .

Figure 4-12 - Load vs. displacement for run with 50\% knockdown of damage initiation strength on trigger.

Figure 4-13 - Deformed plot of (a) original run, (b) modified $D_{\max }$ run, (c) modified bulk viscosity run, and (d) damage initiation strength knockdown run.

Figure 5-1 - Schematic of the 2013 Nosecone. Dimensions shown are in millimeters. 55

Figure 5-2 - Nosecone loaded into MTS test machine prior to quasi-static crush. ….... 57

Figure 5-3 - Load versus displacement for quasi-static crush test of the 2013 nosecone

Figure 5-4 - Energy absorption and deceleration versus displacement from the quasi-static nosecone crush test. 58

Figure 5-5 - Nosecone post quasi-static crush test. 59

Figure 5-6 - Overview of the Test Nosecone mesh. 60

Figure 5-7 - Final deformed shape of nosecone FE model. 63 
Figure 5-8 - Undeformed nosecone shape with fully damaged elements

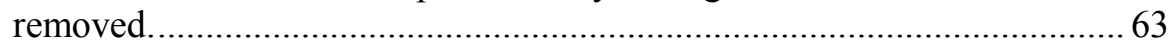

Figure 5-9 - Enveloped fiber compressive damage of the crushed nosecone. ................ 64

Figure 5-10 - Internal and kinetic energies of the nosecone crush model with

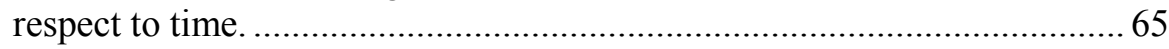

Figure 5-11 - Load versus deflection curves from FEA and testing. ..............................65

Figure 5-12 - Deceleration and energy absorption of nosecone FE model...................... 67

Figure 6-1 - Compact tension (a) and compact compressions (b) test specimens

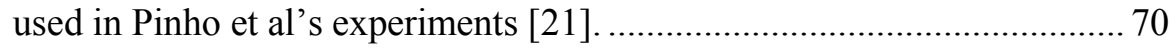




\section{LIST OF UNITS AND VARIBLES}

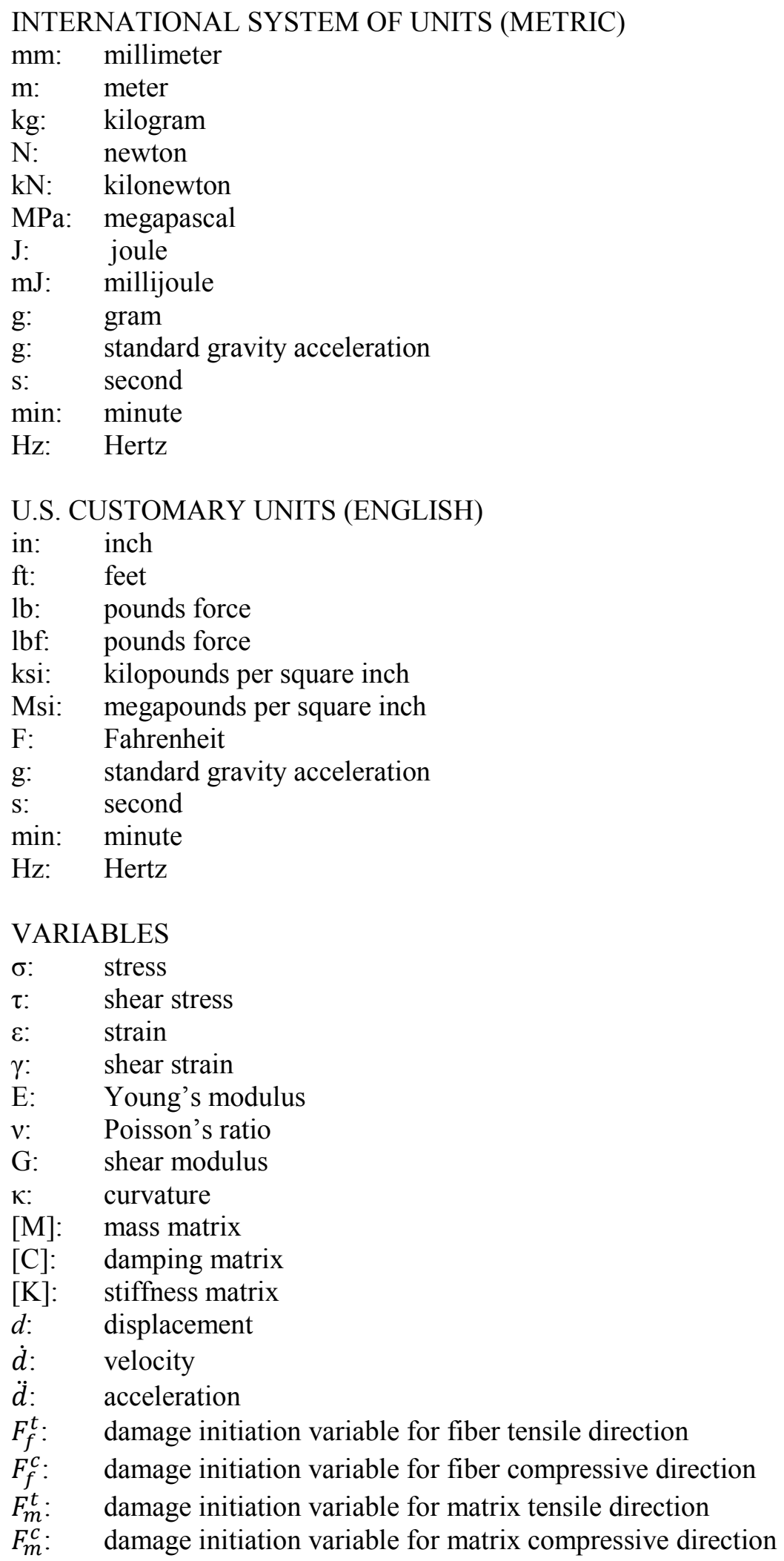


$\begin{array}{ll}\hat{\sigma}: & \text { effective stress } \\ \hat{\tau}: & \text { effective shear stress } \\ X^{T}: & \text { longitudinal tensile strength } \\ X^{C}: & \text { longitudinal compressive strength } \\ Y^{T}: & \text { transverse tensile strength } \\ Y^{C}: & \text { transverse tensile strength } \\ S^{L}: & \text { longitudinal shear strength } \\ S^{T}: & \text { transverse shear strength } \\ d_{f}: & \text { damage variable, fiber } \\ d_{m}: & \text { damage variable, matrix } \\ d_{s}: & \text { damage variable, shear } \\ \mathrm{D}_{\text {max }}: & \text { maximum degradation value } \\ \alpha: & \text { Hashin model operator (defines use of 1973 versus } 1980 \text { model) } \\ \delta_{e q}^{f t}: & \text { equivalent displacement, fiber tensile direction } \\ \delta_{e q}^{f c}: & \text { equivalent displacement, fiber compressive direction } \\ \delta_{e q}^{m t}: & \text { equivalent displacement, matrix tensile direction } \\ \delta_{e q}^{m c}: & \text { equivalent displacement, matrix compressive direction } \\ \delta_{e q}^{f}: & \text { equivalent displacement, at complete failure } \\ \delta_{e q}^{0}: & \text { equivalent displacement, at failure initiation } \\ \sigma_{e q}^{f t}: & \text { equivalent stress, fiber tensile direction } \\ \sigma_{e q}^{f c}: & \text { equivalent stress, fiber compressive direction } \\ \sigma_{e q}^{m t}: & \text { equivalent stress, matrix tensile direction } \\ \sigma_{e q}^{m c}: & \text { equivalent stress, matrix compressive direction } \\ G^{C}: & \text { energy dissipated due to failure } \\ L^{e}: & \text { element characteristic length } \\ c_{d}: & \text { dilatation wave speed } \\ \rho: & \text { density } \\ \mathrm{A}: & \text { area } \\ \mathrm{P}: & \text { load } \\ \mathrm{SCS}: & \text { specific crushing stress } \\ \mathrm{SEA}: & \text { specific energy absorption } \\ \mathrm{CLE}: & \text { crush load efficiency } \\ & \end{array}$ 


\section{CHAPTER 1}

\section{INTRODUCTION}

The Cal Poly Formula SAE (FSAE) team has recently utilized a hybrid carbon fiber monocoque chassis in place of the traditional tubular steel space frame. The design consists of a front monocoque driver cell constructed of carbon fiber skins and a Nomex honeycomb core with a tubular steel space frame attached to the rear of the car that houses the power unit and drivetrain components. The motivation behind this design change was to reduce weight in the chassis by utilizing the high strength-to-weight ratio of carbon fiber while emphasizing the safety of the driver as the monocoque has much better penetration characteristics than its steel tube counterpart. In keeping with the design philosophy, the impact attenuator and nosecone were incorporated into a single part to provide a lightweight solution to absorbing frontal impact energy.

FSAE rules T3.21 and T3.22 [1] state the design criteria for the frontal impact structure. Below are the summarized design criteria:

- Must be at least $200 \mathrm{~mm}$ (7.8 in) long with length aligned with the fore/aft axis of the vehicle

- Must be $100 \mathrm{~mm}$ (3.9 in) high and $200 \mathrm{~mm}$ (7.8 in) wide for at least a length of $200 \mathrm{~mm}$ (7.8 in)

- Must be able to absorb a minimum of $7350 \mathrm{~J}$

- Must be able to stop a $300 \mathrm{~kg}(661 \mathrm{lb})$ vehicle traveling at $7.0 \mathrm{~m} / \mathrm{s}(23.0 \mathrm{ft} / \mathrm{s})$

- Average deceleration may not exceed 20 g's

- Peak deceleration may not exceed 40 g's

Traditionally, the team utilized a SAE pre-approved foam structure that was housed inside of a nosecone fairing. The new design made the nosecone a structural member and 
eliminated the foam impact structure. Carbon fiber reinforced polymer (CFRP) was chosen for the material due to its potential to absorb high amounts of energy from progressive failure modes and high specific strength. Given the complex nature of analysis, the nosecone design was validated by numerous quasi-static crush tests until a layup schedule met the required energy absorption and deceleration requirements. This process was time consuming, labor intensive, and expensive as extensive amounts of material were consumed. A more practical and financially feasible solution would be to utilize computer aided engineering (CAE) tools to develop and validate the design of the nosecone.

\subsection{Literature Review}

Composite materials have been in the automotive sector in the application of motorsport for quite some time. In Formula 1, the first carbon fiber composite chassis was introduced in 1980 by the McLaren team [2]. Though designers had concerns of the safety of composite chassis, the advantages of CFRP chassis were realized in 1981 when driver John Watson violently crashed his McLaren at the Italian Grand Prix and walked away unscathed. Since then, CFRP's have been utilized extensively in Formula 1 and motorsport.

There have been numerous studies conducted in an effort to implement CFRP's into crash structures. Teams from Formula 1 have conducted extensive research into CFRP impact structures in order to produce high performing lightweight and safe chassis that meet the strict impact requirements set forth by the Fédération Internationale de I'Automodile (FIA), the governing body for Formula 1 racing [2] [3] [4]. These papers all exhibit the importance of progressive failure in energy absorbing structures. They also show that the main failure modes for composite structures are fiber and matrix fracture and a majority of the energy absorption is from brittle material failure. 
Moving from professional racing applications, Formula SAE teams have also implemented composite impact structures onto their vehicles [5]. Obradovic et al. implemented a composite frontal impact structure on Polytechnic of Turin's FSAE car. They utilized an analytical model based on work-energy of failure mechanisms, a finite element model, and experimental testing to design and validate their impact attenuator. They were able to show good agreement with their analysis methods, more importantly achieving accuracy to within about $10 \%$ between experimental quasi-static crushing and finite element analysis.

Composite materials, especially carbon fiber, can be very well suited for impact structures for increasing crash safety. In contrast to metallic impact structures that involve plastic deformation, the high stiffness of carbon fiber does not allow that material to exceed its elastic limit as illustrated below in Figure 1-1 and Figure 1-2. This serves to transmit the load from the point of impact further into the structure [2]. Once the load in the local area of impact has exceeded the absolute strength of the material, failure is initiated and the composite progressively tears itself to pieces. The energy of the impact is absorbed via fracture mechanisms since there is no yielding of the material. The primary energy absorption mechanisms in CFRP's are cracking and fracture of fibers, matrix fracture, fiber pull out from the matrix, and delamination of layers in the structure.
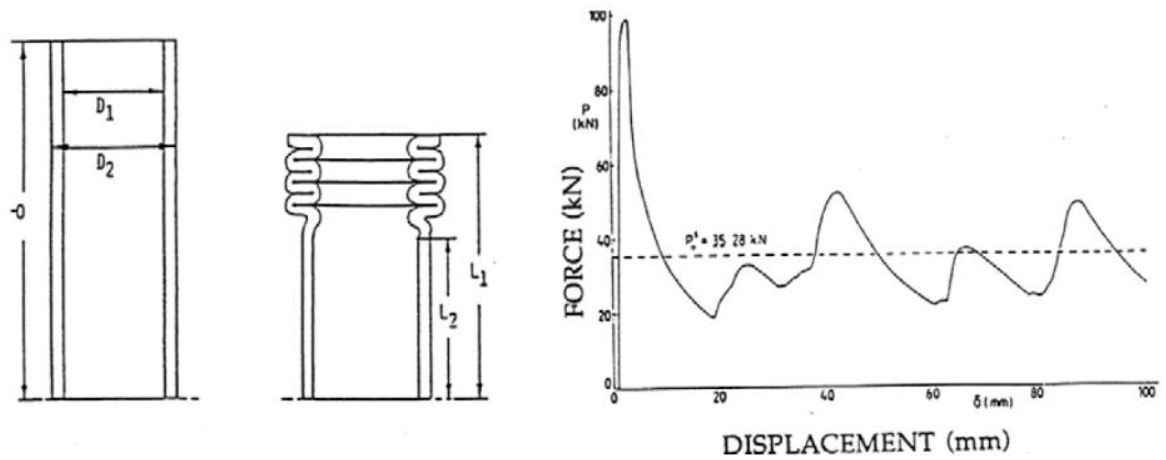

Figure 1-1 - Energry absorption by metallic impact structure [2]. 

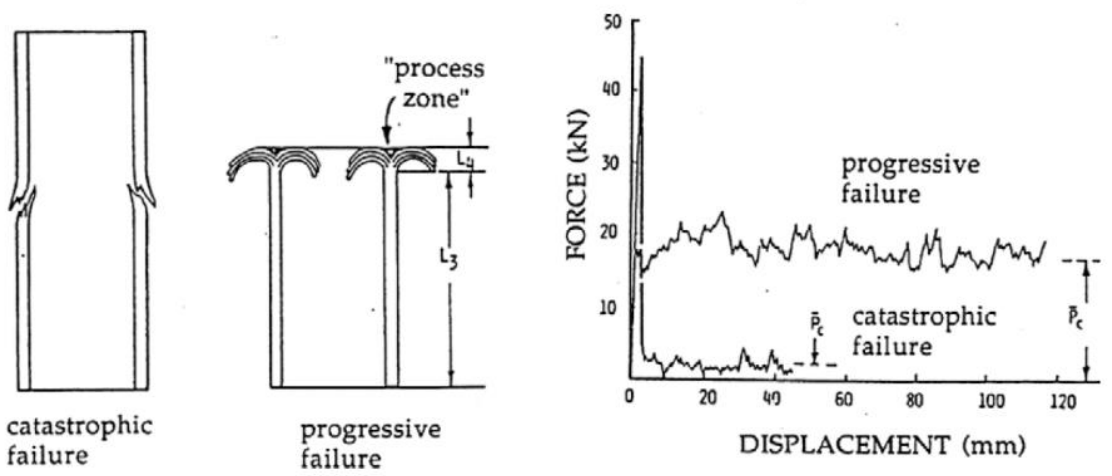

\section{Figure 1-2 - Energy absorption of composite impact structure showing catastrophic and progressive failure [2].}

Composite crash structures have the potential to have higher energy absorption per unit weight while reducing the noise and vibrations in the structure compared to their metallic counterparts [6]. They can be tailored very robustly by altering parameters such as fiber type, matrix type, fiber architecture, structure geometry, manufacturing process, and fiber volume fraction. Because of the large amount of design variables, high expense of physical and experimental testing, and development of advanced finite element codes, numerical methods are an attractive choice for design validation and optimization of these structures.

Numerous methods have been developed in order to model the progressive damage of CFRP structures. One of the more common models is the Hashin failure criterion described in Chapter 2. This model defines failure criterion for four failure modes; fiber tension and compression, and matrix tension and compression [7]. After initiation, the stiffness matrix is degraded according to a fracture energy method [8]. Other more recent models take into account more complex failure modes. Pinho et al. developed a similar failure criterion to Hashin, except the fiber and matrix compressive initiation takes into account the rotation of stress in the fracture plane caused by fiber kinking [9] [10].This better predicts fiber kinking and transverse compression failures. Additionally, this model also takes into account an inter-laminar shear stress failure. While the accuracy of this model proved to be high, its complexity leads to more material constants that need to be defined. In addition to the basic strength and stiffness values, 
fracture toughness for inter-laminar (longitudinal and transverse), intra-laminar, fiber tensile, and fiber kinking as well as the fracture angle for pure transverse compression are required. This leads to additional testing to determine these values.

In addition to development of finite element models for crash structures, numerous optimization methods have been studied. Chen utilized a robust genetic algorithm to optimize the design of impact structures [11]. He found that although the genetic algorithm could be applied to the impact structure optimization problem, the instability of the explicit finite element (FE) model utilized made achieving a global optimum point very difficult. Forsberg and Nilsson explored the optimization of automobile impact structures using classical response surface methodology (CRSM) and Kriging theory to approximate the objective functions [12]. They found that Kriging theory converged more quickly than CRSM, but is heavily influenced by initial parameters and may converge to a local rather than a global optimum. Lanzi, Castelletti, and Anghileri approached the optimization of the impact structure shape using a combination of Radial Basis Functions (RBFs) and Genetic Algorithms [13]. They built the response surface of the objective function by assuming a bilinear load vs. deflection curve derived from FE analysis of a sample of conical impact structure shapes and interpolating them to a wide range of geometries using RBFs. Then, a Genetic Algorithm was used on the response surface to determine optimal geometries. They found that this method was computationally less expensive than running a finite element model (FEM) for each design case. Additionally, they found that conical shaped impact structures exhibit better specific energy absorption in vertical and off-axis impact with weight savings of up to $45 \%$ when compared with cylindrical impact structures. 


\subsection{Goals}

The goal of this thesis was to develop a process to analyze a CFRP impact attenuator using Abaqus CAE finite element analysis (FEA) software. This was done by first exploring the Hashin damage model utilized by Abaqus. Next, a FE damage model developed previously [14] was validated by means of laboratory testing. Finally, the FE damage model was applied to the geometry of the 2013 nosecone and compared to the quasi-static crush test. 


\section{CHAPTER 2}

\section{OVERVIEW OF THE MODELING PROCESS}

\subsection{CFRP Design and Analysis}

Carbon fiber reinforced polymer (CFRP) is an extremely lightweight and strong fiber reinforced polymer. The material can be made from chopped, short fibers or long continuous fibers. This study will focus on the design of continuous CFRPs, since that was the material utilized in the manufacture of the nosecone. There are many types of continuous CFRP, however the most common are unidirectional and bidirectional. Unidirectional CFRP's consist of long carbon fibers oriented in the same direction surrounded by a matrix of resin or other type of polymer. Bidirectional CFRP's consist of carbon fibers woven together, typically perpendicular to one another, surrounded by a polymer matrix. This material is very strong when oriented in the fiber direction. However, because of the directionality of the fibers, the material is considered anisotropic and must be analyzed as such.

Fiber composites generally fall under a category of anisotropic materials called orthotropic material. An orthotropic material is one that has two or three planes of rotational symmetry with regards to its elastic properties. This reduces the number of independent elastic constants required for analysis since the invariance of the elastic properties must be satisfied [15]. The elastic constants of an orthotropic material can be expressed in array form as shown in (2-1).

$$
E_{i j k l}=\left[\begin{array}{cccccc}
E_{1111} & E_{1122} & E_{1133} & 0 & 0 & 0 \\
E_{1122} & E_{2222} & E_{2233} & 0 & 0 & 0 \\
E_{1133} & E_{2233} & E_{3333} & 0 & 0 & 0 \\
0 & 0 & 0 & E_{2323} & 0 & 0 \\
0 & 0 & 0 & 0 & E_{1313} & 0 \\
0 & 0 & 0 & 0 & 0 & E_{1212}
\end{array}\right]
$$


It is easier and more typical to write the elastic matrix in contracted notation as the stiffness matrix $C_{i j}$. In indicial notation and matrix form respectively, the stress strain relationship can be expressed in (2-2) and (2-3), where $i, j=1,2,3,4,5,6$.

$$
\begin{gathered}
\sigma_{i}=C_{i j} \varepsilon_{j} \\
\left\{\begin{array}{l}
\sigma_{1} \\
\sigma_{2} \\
\sigma_{3} \\
\tau_{23} \\
\tau_{13} \\
\tau_{12}
\end{array}\right\}=\left[\begin{array}{cccccc}
C_{11} & C_{12} & C_{13} & 0 & 0 & 0 \\
C_{12} & C_{22} & C_{23} & 0 & 0 & 0 \\
C_{13} & C_{23} & C_{33} & 0 & 0 & 0 \\
0 & 0 & 0 & C_{44} & 0 & 0 \\
0 & 0 & 0 & 0 & C_{55} & 0 \\
0 & 0 & 0 & 0 & 0 & C_{66}
\end{array}\right]\left\{\begin{array}{l}
\varepsilon_{1} \\
\varepsilon_{2} \\
\varepsilon_{3} \\
\gamma_{23} \\
\gamma_{13} \\
\gamma_{12}
\end{array}\right\}
\end{gathered}
$$

For most structural applications, composites are loaded in the plane of the laminate. This is called a plane stress loading condition and assumes all out-of-plane stress components are zero [15]. The stress-strain relationships can then be expressed using the stiffness matrix $[Q]$ as expressed below in (2-4).

$$
\left\{\begin{array}{l}
\sigma_{1} \\
\sigma_{2} \\
\tau_{12}
\end{array}\right\}=\left[\begin{array}{ccc}
Q_{11} & Q_{12} & 0 \\
Q_{12} & Q_{22} & 0 \\
0 & 0 & Q_{66}
\end{array}\right]\left\{\begin{array}{l}
\varepsilon_{1} \\
\varepsilon_{2} \\
\gamma_{12}
\end{array}\right\}
$$

(2-4) can be used for a single lamina. To obtain the stress-strain relationship in the whole laminate, the stiffness matrix must be integrated through the thickness of the composite. This is shown in (2-5) below. 


$$
\left\{\begin{array}{l}
N_{x} \\
N_{y} \\
N_{x y} \\
M_{x} \\
M_{y} \\
M_{x y}
\end{array}\right\}=\left[\begin{array}{c:c}
A_{i j} & B_{i j} \\
\hdashline B_{i j} & D_{i j}
\end{array}\right]\left\{\begin{array}{l}
\varepsilon_{x} \\
\varepsilon_{y} \\
\varepsilon_{x y} \\
\kappa_{x} \\
\kappa_{y} \\
\kappa_{x y}
\end{array}\right\}
$$

Where

$$
\begin{array}{r}
A_{i j}=\sum_{k=1}^{N}\left(\overline{Q_{i j}}\right)\left(z_{k}-z_{k-1}\right) \\
B_{i j}=\frac{1}{2} \sum_{k=1}^{N}\left(\overline{Q_{i j}}\right)\left(z_{k}^{2}-z_{k-1}^{2}\right) \\
D_{i j}=\frac{1}{3} \sum_{k=1}^{N}\left(\overline{Q_{i j}}\right)\left(z_{k}^{3}-z_{k-1}^{3}\right)
\end{array}
$$

$k$ is the layer number, $N$ is the total number of layers, $\bar{Q}_{i j}$ is the transformed stiffness term, and $z$ is the distance from the mid-plane. The equations above are used by Abaqus in the definition of lamina type materials to develop the material tensor.

\subsection{Finite Element Analysis Theory}

In order to more efficiently analyze composite parts, the finite element method is utilized. The basic theory behind the finite element method is to discretize a body into small parts or elements and assume a displacement field within each element. Then, by applying boundary conditions such as loads and known displacements, the unknown displacements can be solved for by equation (2-9) where $\{d\}$ is the displacement for each degree of freedom for each node of the element, $[K]$ is the stiffness matrix for the system, and $\{F\}$ is the force applied to each degree of freedom for each node.

$$
\{d\}=[K]^{-1}\{F\}
$$


As shown above, this method of solving for the nodal displacements requires the inversion of the stiffness matrix. For small displacement, static problems, converging to a solution is not very difficult using this method. For dynamic problems Abaqus CAE can handle the problem using two different methods: implicit or explicit integration.

\subsubsection{Implicit versus Explicit Integration in Transient Dynamic FEA}

Implicit integration is done by means of the Linear Acceleration Method or Newmarkbeta method and is called Abaqus/Standard within Abaqus CAE. The equation to solve for the unknown displacements is shown below in $(2-10)$, where $[M]$ is the mass matrix, $[C]$ is the dampening matrix, $[K]$ is the stiffness matrix, $\{d\}$ is the nodal displacements, $\{\dot{d}\}$ is the nodal velocities, $\{\ddot{d}\}$ is the nodal accelerations, $n$ is the current time step, $\Delta t$ is the change in time for the time step, $\beta$ is 0.25 and $\gamma$ is 0.5 [16].

$$
\begin{aligned}
\left(\frac{1}{\beta(\Delta t)^{2}}[M]+\frac{\gamma}{\beta \Delta t}[C]+[K]\right)\left\{d_{n+1}\right\} & =\left\{F_{n+1}\right\} \\
& \left.+[M]\left(\frac{1}{\beta(\Delta t)^{2}}\left\{d_{n}\right\}+\frac{1}{\beta \Delta t}\left\{\dot{d}_{n}\right\}+\left(\frac{1}{2 \beta}-1\right) \ddot{d}_{n}\right\}\right) \\
& +[C\}\left(\frac{\gamma}{\beta \Delta t}\left\{d_{n}\right\}+\left(\frac{\gamma}{\beta}-1\right)\left\{\dot{d}_{n}\right\}+\Delta t\left(\frac{\gamma}{2 \beta}-1\right)\left\{\dot{d}_{n}\right\}\right)
\end{aligned}
$$

More simply, (2-10) can be rewritten as (2-11) below.

$$
[\bar{K}]\left\{d_{n+1}\right\}=\left\{F_{e f f}\right\}
$$

This form of the implicit method closely resembles that of the general static FEA solution. Essentially, the modified stiffness matrix $[\bar{K}]$ must be inverted to determine the nodal displacements at each time step. For small systems, this method is computationally efficient and works well for analyses that have long durations. Additionally, it is unconditionally stable for all $\Delta t$; however it is only accurate for smaller time increments. The implicit method becomes a problem when systems become large as inverting the stiffness matrix becomes computationally 
taxing and convergence to a solution is difficult. To solve this problem, an explicit integration method should be utilized.

Explicit integration is done by means of the Central Difference Method and is called Abaqus/Explicit within Abaqus CAE. The equation to solve for the unknown displacements is shown below in (2-12). Note that the explicit method solves for the system state at the next time step based on the current system state.

$$
\begin{aligned}
\left(\frac{1}{(\Delta t)^{2}}[M]+\frac{1}{2 \Delta t}[C]\right)\left\{d_{n+1}\right\} & \left\{F_{n}\right\}-[K]\left\{d_{n}\right\} \\
& +\frac{2}{(\Delta t)^{2}}[M]\left\{d_{n}\right\} \\
& -\left(\frac{1}{(\Delta t)^{2}}[M]-\frac{1}{2 \Delta t}[C]\right)\left\{d_{n-1}\right\}
\end{aligned}
$$

The explicit method is conditionally stable and is dependent on small time increments; however it is a more efficient solver for large dynamic systems as the stiffness matrix does not need to be inverted. Therefore, for a lumped mass system (i.e. [M] and [C] are diagonal), each term in $\left\{d_{n+l}\right\}$ can be explicitly solved. It is best suited for impact or short duration dynamic analysis. It is because of this, Abaqus/Explicit was chosen as the integration method for the FE analysis.

\subsection{Modeling Damage of Fiber Reinforced Composites in FEA}

In order to simulate the progressive damage of composites, a damage model must be defined in the material definition. Damage is characterized by the degradation of material stiffness [8]. Abaqus handles the damage of fiber reinforced composites by specifying an undamaged linear elastic response, followed by a damage initiation criterion, and concluded by a damage evolution response. The undamaged portion of the model is handled by the linear elastic definition of the material as described in Section 2.1. The damage initiation criterion is defined based on the work of Hashin and Rotem. 


\subsubsection{Damage Initiation}

The onset of damage of a material point is determined by damage initiation criteria based on Hashin's theory [8]. The criterion takes into account four different damage initiation mechanisms: fiber tension, fiber compression, matrix tension, and matrix compression. The criterion are given below for fiber tension (2-13) and compression (2-14) and matrix tension (2-15) and compression (2-16),

$$
\begin{gathered}
F_{f}^{t}=\left(\frac{\hat{\sigma}_{11}}{X^{T}}\right)^{2}+\alpha\left(\frac{\hat{\tau}_{12}}{S^{L}}\right)^{2} \\
F_{f}^{c}=\left(\frac{\hat{\sigma}_{11}}{X^{C}}\right)^{2} \\
F_{m}^{t}=\left(\frac{\hat{\sigma}_{22}}{Y^{T}}\right)^{2}+\left(\frac{\hat{\tau}_{12}}{S^{L}}\right)^{2} \\
F_{m}^{c}=\left(\frac{\hat{\sigma}_{22}}{2 S^{T}}\right)^{2}+\left[\left(\frac{Y^{C}}{2 S^{T}}\right)^{2}-1\right] \frac{\hat{\sigma}_{22}}{Y^{C}}+\left(\frac{\hat{\tau}_{12}}{S^{L}}\right)^{2}
\end{gathered}
$$

where $X^{T}$ is longitudinal tensile strength, $X^{C}$ is longitudinal compressive strength, $Y^{T}$ is transverse tensile strength, $Y^{C}$ is transverse compressive strength, $S^{L}$ is longitudinal shear strength, $S^{T}$ is transverse shear strength, $\alpha$ is the coefficient for shear contribution to the tensile initiation criterion, and $\hat{\sigma}_{11}, \hat{\sigma}_{22}$, and $\hat{\tau}_{12}$ are the components of the equivalent stress vector.

The equivalent stress vector is computed from

$$
\{\hat{\sigma}\}=[M]\{\sigma\}
$$

where $\{\sigma\}$ is true stress and $[M]$ is the damage operator. The damage operator is defined as 


$$
[M]=\left[\begin{array}{ccc}
\frac{1}{\left(1-d_{f}\right)} & 0 & 0 \\
0 & \frac{1}{\left(1-d_{m}\right)} & 0 \\
0 & 0 & \frac{1}{\left(1-d_{s}\right)}
\end{array}\right]
$$

where $d_{f}, d_{m}$, and $d_{s}$ are internal damage variables that characterize fiber, matrix, and shear damage. Prior to any damage initiation, $[M]$ is equal to the identity matrix; therefore the equivalent stresses are equal to the true stresses. Once damage is initiated and evolution has occurred in at least one mode, the damage operator becomes significant in determining the damage criteria for other modes.

It should be noted that Abaqus has two damage models incorporated into the software: the Hashin and Rotem 1973 model and the Hashin 1980 model. The difference between the two models is the 1980 model incorporates a shear interaction in the fiber tension criteria. The way a model is determined is through the $\alpha$ coefficient. By setting $\alpha$ to 0.0 and $S^{T}=Y^{C} / 2$ the 1973 model is specified. By setting $\alpha$ to 1.0 , the 1980 model is specified.

\subsubsection{Damage Evolution}

After damage is initiated, the damage evolution model controls the material response. The response of the material post damage follows (2-19) where $\left[C_{d}\right]$ is the damaged elasticity matrix specified in (2-20) and $D$ is specified by (2-21).

$$
\begin{gathered}
\{\sigma\}=\left[C_{d}\right]\{\varepsilon\} \\
{\left[C_{d}\right]=\frac{1}{D}\left[\begin{array}{ccc}
\left(1-d_{f}\right) E_{1} & \left(1-d_{f}\right)\left(1-d_{m}\right) v_{21} E_{1} & 0 \\
\left(1-d_{f}\right)\left(1-d_{m}\right) v_{12} E_{2} & \left(1-d_{m}\right) E_{2} & 0 \\
0 & 0 & \left(1-d_{s}\right) G D
\end{array}\right]} \\
D=1-\left(1-d_{f}\right)\left(1-d_{m}\right) v_{12} v_{21}
\end{gathered}
$$


The terms $d_{f}, d_{m}$, and $d_{s}$ are damage state variables associated with fiber, matrix, and shear damage respectively. $\left[C_{d}\right]$ is essentially the plane stress stiffness matrix $[Q]$ modified by the damage state variables. As the damage in each direction increases, the material is softened accordingly, thereby capturing the damaged response.

In order to determine the evolution of the damage variables for each mode, Abaqus uses a modified stress-strain relation. First, a characteristic length is introduced into the formulation changing the constitutive law into a stress-displacement relation. This alleviates problems associated with mesh dependency during material softening. The damage variables evolve such that the stress-displacement behaves as a bilinear relationship as shown below in Figure 2-1 for each of the four failure modes. The positive slope up to a displacement of $\delta_{e q}^{0}$ represents the undamaged, linear elastic response of the material and the onset of damage initiation. The negative slope represents the post damaged behavior.

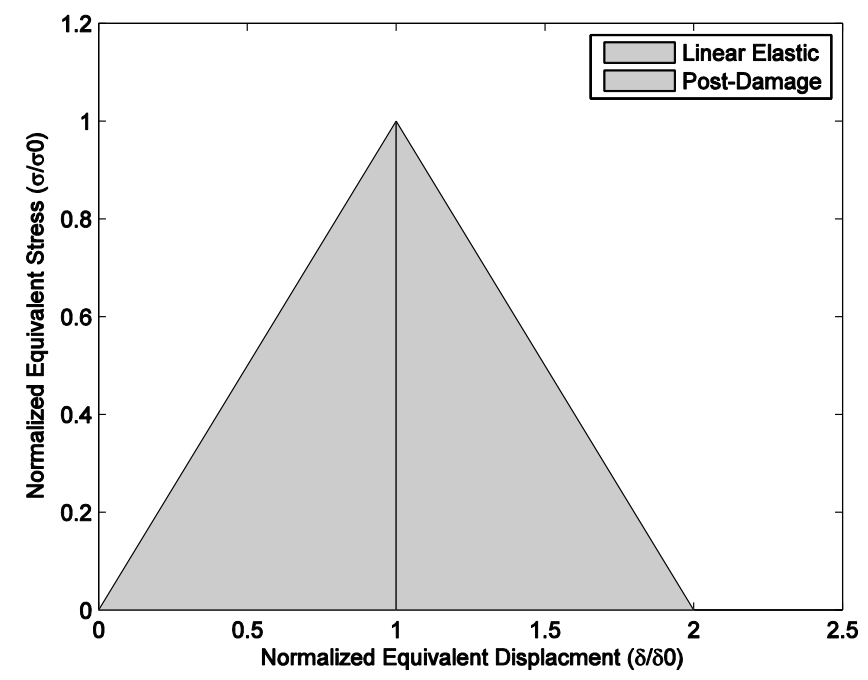

Figure 2-1 - Equivalent stress versus equivalent displacement. Note that the figure above shows the material completely fails at twice the displacement of damage initiation. This is not always true for all materials. 
The equivalent stress and displacement are determined by the following equations for fiber tension, fiber compression, matrix tension, and matrix compression where $L^{c}$ is the characteristic length and the \langle\rangle is the Macaulay operator defined as $\langle\beta\rangle=(\beta+|\beta|) / 2$.

$$
\begin{gathered}
\delta_{e q}^{f t}=L^{c} \sqrt{\left\langle\varepsilon_{11}\right\rangle^{2}+\alpha \varepsilon_{12}^{2}} \\
\sigma_{e q}^{f t}=\frac{\left\langle\sigma_{11}\right\rangle\left\langle\varepsilon_{11}\right\rangle+\alpha \tau_{12} \varepsilon_{12}}{\delta_{e q}^{f t} / L^{c}} \\
\delta_{e q}^{f c}=L^{c}\left\langle-\varepsilon_{11}\right\rangle \\
\sigma_{e q}^{f c}=\frac{\left\langle-\sigma_{11}\right\rangle\left\langle-\varepsilon_{11}\right\rangle}{\delta_{e q}^{f c} / L^{c}} \\
\delta_{e q}^{m t}=L^{c} \sqrt{\left\langle\varepsilon_{22}\right\rangle^{2}+\varepsilon_{12}^{2}} \\
\sigma_{e q}^{m t}=\frac{\left\langle\sigma_{22}\right\rangle\left\langle\varepsilon_{22}\right\rangle+\tau_{12} \varepsilon_{12}}{\delta_{e q}^{m t} / L^{c}} \\
\sigma_{e q}^{m c}=\frac{\left\langle-\sigma_{22}\right\rangle\left\langle-\varepsilon_{22}\right\rangle+\tau_{12} \varepsilon_{12}}{\delta_{e q}^{m c} / L^{c}} \\
\delta_{e q}^{m c}=L^{c} \sqrt{\left\langle-\varepsilon_{22}\right\rangle^{2}+\varepsilon_{12}^{2}} \\
\left\langle\begin{array}{l}
{ }_{e q} \\
{ }_{e q}
\end{array}\right.
\end{gathered}
$$

The characteristic length is based on the element's geometry and formulation. In the case for CFRP composites where shell elements are used, it is the characteristic length in the reference surface which is the square root of the area.

In addition to determining equivalent stresses and displacements, a damage variable needs to be determined. The damage variable for any mode is given by (2-30) where the $f$ and 0 superscripts denote complete failure and damage initiation respectively. The relationship between the damage variable and equivalent displacement is shown below in Figure 2-2. 


$$
d=\frac{\delta_{e q}^{f}\left(\delta_{e q}-\delta_{e q}^{0}\right)}{\delta_{e q}\left(\delta_{e q}^{f}-\delta_{e q}^{0}\right)}
$$

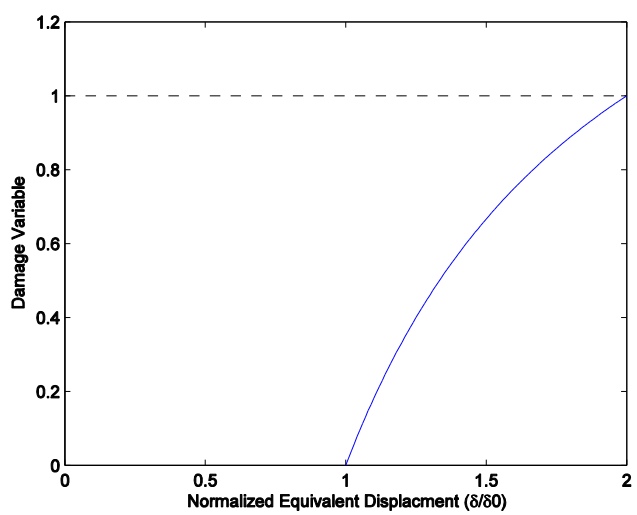

(a)

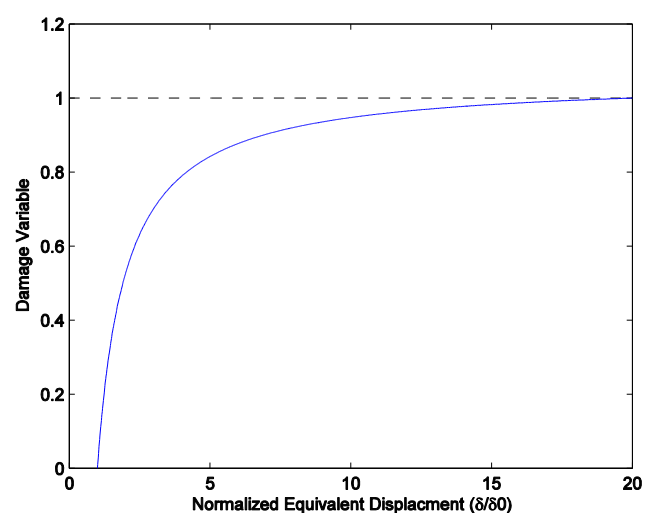

(b)

Figure 2-2 - Evolution of the damage variable with failure displacement $\delta_{e q}^{f}=2 \delta_{e q}^{0}$ (a) and $20 \delta_{e q}^{0}$ (b).

Finally, $\delta_{e q}^{f}$ needs to be specified. Abaqus determines the equivalent failure displacement using energy dissipated due to failure for each mode $\left(G^{C}\right)$, as specified by the user. This corresponds to the area under the equivalent stress-equivalent displacement curve. This is illustrated below in Figure 2-3 by triangle OAB. Note this is the same as the area under the stressstrain curve for each failure mode.

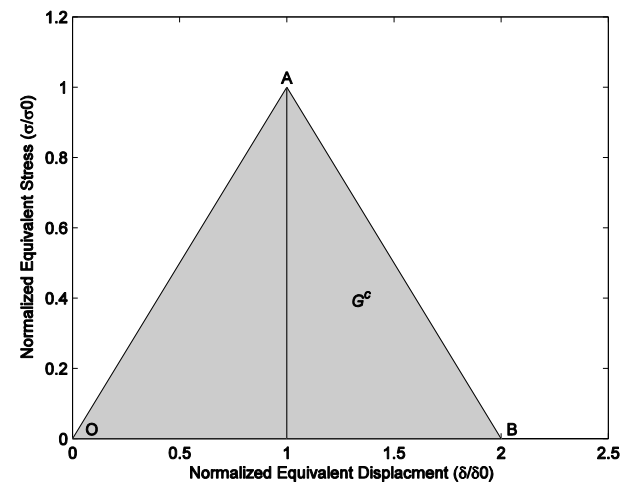

(a)

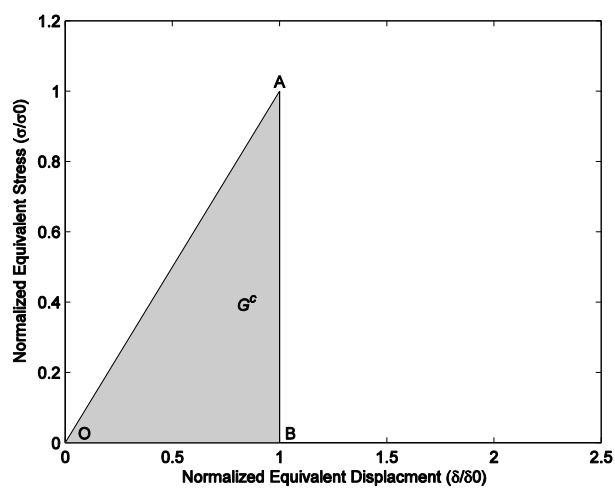

(b)

Figure 2-3 - Definition of failure energy used to determine $\delta_{e q}^{f}$. A typical fiber reinforced polymer composite is shown in (a). The assumed CFRP stress-displacement curve is shown in (b). 
Given the brittle nature of carbon fiber composites, the stress-displacement response is assumed to take the form illustrated in Figure 2-3(b). Essentially, once failure is initiated in a particular mode, the material cannot handle any load in that direction. To determine $G^{C},(2-31)$ was utilized using the elastic modulus $E$ and failure strength $\sigma^{f}$ for the $i^{\text {th }}$ mode.

$$
G_{i}^{C}=\frac{\left(\sigma_{i}^{f}\right)^{2}}{2 E_{i}}
$$

Abaqus also gives the option of setting the maximum degradation variable (i.e. the damage variable), $D_{\max }$. By setting $D_{\max }$, the user can specify when the element integration point is considered completely damaged. For example, if the user set $D_{\max }$ to a value of 0.85 , then the element integration point would be considered completely damaged when the material is $85 \%$ damaged at that point. This can be used to ensure element deletion and is a parameter that can be tuned to help the model match reality. Note that the $D_{\max }$ variable sets the maximum value of the damage parameter for all failure modes.

\subsubsection{Element Removal}

Abaqus has the option of removing elements that have been completely damaged from the visualization of the model. Note that these elements still exist within the model but can be hidden from view (see Figure 2-4). By default, this is done by way of the STATUS field variable. In order for element removal to function, the user must specify the STATUS variable as a field variable so Abaqus can track which elements are active. The STATUS variable can hold two values; either a 1 or a 0 . By default, every element has a STATUS value of 1 . In Abaqus/Standard, that value switches to 0 when all damage variables have reached $D_{\max }$ for all failure modes at all material points within the element. In Abaqus/Explicit, the STATUS is set to 0 when the damage variable associated with fiber failure (either tensile or compressive) reaches $D_{\max }$ for all section points at one integration point within the element. For example, for shell 
elements the element is removed when $D_{\max }$ is reached for fiber failure for all through-thethickness section points at an integration point.

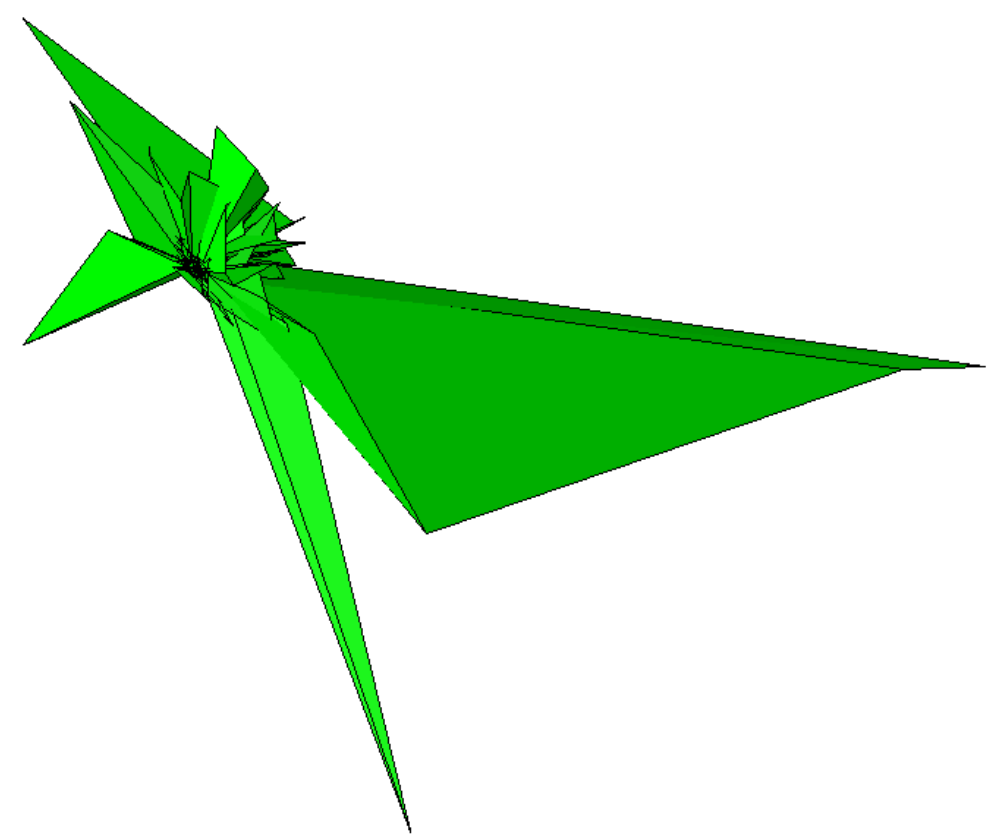

Figure 2-4 - Example of deformed model with status variable not utilized in the visualizer.

While the STATUS field variable is the default status variable used for element removal in the Abaqus visualizer, other field variables can be utilized to remove elements from the visualization. Any field variable such as stress (S), damage criteria (i.e. HSNFTCRT), and damage variables (i.e. DAMAGEFT) can be used to activate and deactivate elements [8]. For the Hashin damage model, the STATUS field variable offers the best element removal scheme and should be utilized for viewing results.

\subsubsection{Damage Stabilization}

Material models that include stiffness degradation and softening behavior often lead to convergence issues with implicit type solvers [8]. This can be overcome by imposing a viscous regularization scheme, causing the tangent stiffness matrix of the material to be positive for small time increments. In this scheme, a viscous damage variable, $d_{v}$, is defined by (2-32), 


$$
\dot{d}_{v}=\frac{1}{\eta}\left(d-d_{v}\right)
$$

where $\eta$ is the viscosity coefficient representing the relaxation time of the viscous system and $d$ is the damage variable evaluated in the inviscid backbone model [8]. The damage response is given in (2-19) where the damaged elasticity matrix, $\left[C_{d}\right]$, is evaluated using viscous values of damage variables for each failure mode. The viscosity parameter should be small compared to the characteristic time increment. The idea behind damage stabilization is that the solution of the viscous system relaxes to that of the inviscid case as the ratio of time to the viscosity parameter $(t / \eta)$ approaches infinity.

Damage stabilization can be used for Abaqus/Explicit as well. In the explicit solver, the viscous regularization slows down the rate of increase of damage and leads to increased fracture energy with increasing deformation rates. This can be used as a method of modeling rate dependent material behavior.

\subsection{Quasi-Static Considerations with Explicit Dynamic FEA}

The problems that are attempted to be modeled herein are classified as quasi-static. These problems involve large deformation and usually involve complex contact conditions. While the tension tests in the following section do not involve contact, the tube crushing and nosecone models do. Thus, because of these conditions it is more computationally efficient to use the explicit solver even though it solves for dynamic equilibrium (rather than static equilibrium with implicit). However, because explicit dynamics solves for dynamic equilibrium where inertial forces play a dominant role, special considerations must be made when modeling quasi-static problems.

If a quasi-static problem were to be modeled in its natural time period, an excessive amount of time increments would be required for the solution thus making it computationally impractical. Therefore, it is necessary to artificially increase the speed of the process to obtain an 
practical solution. This is typically done in two ways: load rate scaling and mass scaling. Both of these techniques reduce the number of time increments thus reducing the time it takes to reach a solution.

\subsubsection{Load Rate Scaling}

By increasing the rate at which loads are applied to the structure, the overall time period of the step is decreased, thus decreasing the total computation time. The dominant response of the structure during a quasi-static analysis will be the first structural mode [17]. To determine the impact velocity, first the fundamental frequency of the structure needs to be determined. From that, the corresponding time period $(T)$ can be determined. Finally, by estimating the global impact deflection $(D)$, the impact velocity can be determined by using (2-33).

$$
V=D / T
$$

Note that Abaqus recommends that the impact velocity should not exceed $1 \%$ of the wave speed of the material.

When artificially increasing the loading rate, localized effects can alter the results of the model. One such effect is a steep initial slope in the load versus displacement curve. This is caused by inertial effects causing non-structural resistance to initial deformation. Care must be taken when increasing load rates to ensure that the response of the system is truly structural and that dynamic effects are minimal.

\subsubsection{Mass Scaling}

Increasing the load rate on a model will artificially increase the strain rates of the material by the same factor. This may be undesirable (e.g. if material is strain-rate dependent) and the model may need to be analyzed in its natural time period. This can be accomplished by mass scaling. 
An estimate of the stability limit in an explicit dynamics procedure can by expressed as (2-34) where $L^{e}$ is the smallest characteristic element length and $c_{d}$ is the dilatation wave speed [17].

$$
\Delta t=\left(\frac{L^{e}}{c_{d}}\right)
$$

For simplicity, the dilatation wave speed in a linear elastic material with a Poisson's ratio equal to zero is given as [17].

$$
c_{d}=\sqrt{\frac{E}{\rho}}
$$

As shown, if the density is increased by a factor of $f^{2}$, then the dilatation wave speed is decreased by a factor of $f$. This in turn increases the stable time step by a factor of $f$. Therefore, it can be concluded that a factor of $f^{2}$ increase in density will result in a factor of $f$ increase in stable time increment. This reduces the number of time increments required for the solution and thus makes the model more economical.

\subsubsection{Smooth Step Load Application}

In order to reduce stiffening from inertial effects and stress wave propagation, loads must be applied gradually. By default, Abaqus/Explicit applies loads instantaneously and remains constant throughout the step. Velocity boundary conditions behave in the same way. To alleviate this problem, a Smooth Step amplitude curve can be defined in Abaqus. This creates a fifth order polynomial transition between two amplitude values such that the first and second time derivatives are zero at the beginning and end of the transition [17]. The amplitude values act as a scaling factor applied to the boundary conditions it is assigned to. Figure 2-5 and Figure 2-6 below illustrates how the Smooth Step amplitude curve works. 


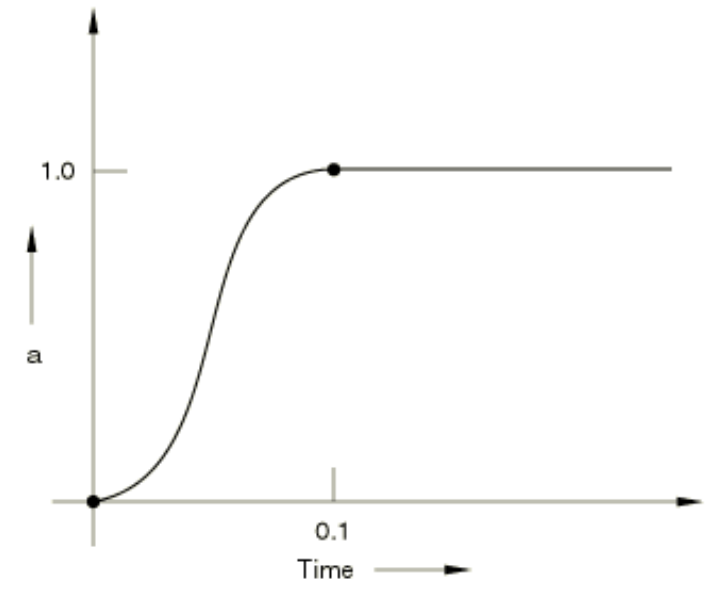

(a)

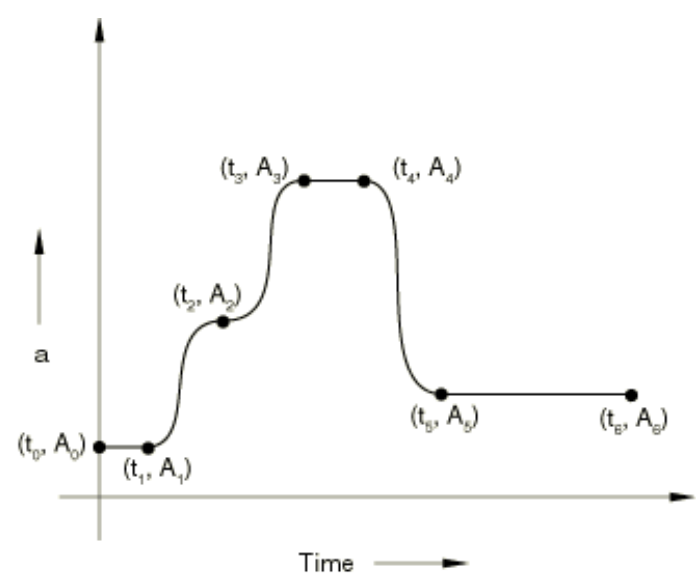

(b)

Figure 2-5 - Smooth step amplitude curves for (a) one defined amplitude point and (b) multiple defined amplitude points [17].

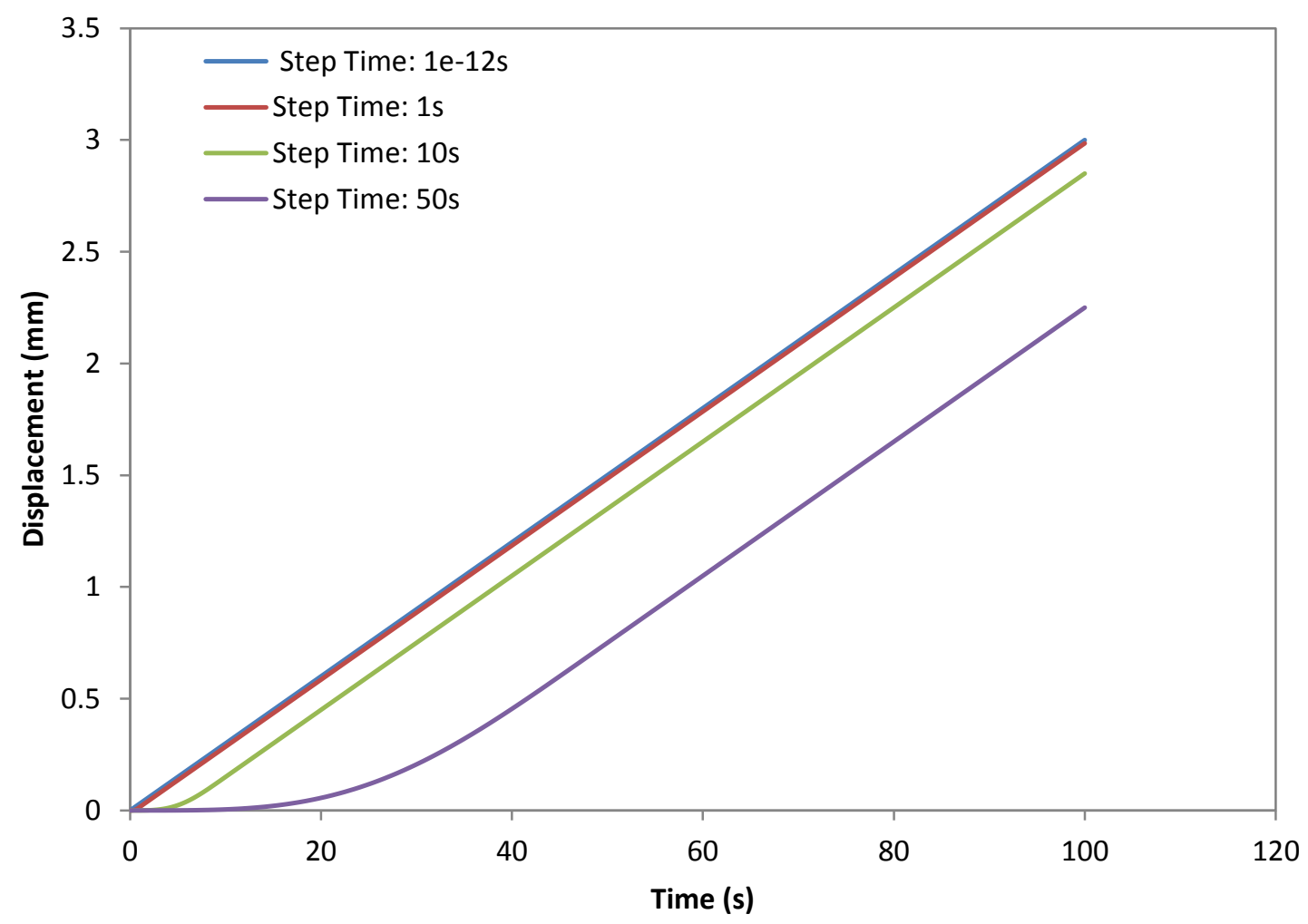

Figure 2-6 - Impact displacement curves with various single amplitude point Smooth Step curves applied to impact velocity boundary conditions [17]. 


\subsubsection{Energy Balance}

In order to evaluate whether or not the model is yielding an appropriate quasi-static response, an energy balance equation can be utilized. At its core, a physical test is quasi-static if the work from the external forces equals the internal energy of the specimen. Therefore, the kinetic energy of the deformable material should not exceed a small percentage of its internal energy throughout the procedure. Abaqus suggests that a small percentage typically means $1 \%$ 5\% [17]. An ideal quasi-static energy curve is shown below in Figure 2-7. Usually, it is not possible to achieve static energy balance early in the analysis since the deformable body will be moving before any significant deformation, but this can be somewhat alleviated by using Smooth Step amplitude curves as discussed above. Additionally, the kinetic energies of rigid bodies should not be considered in the energy balance.

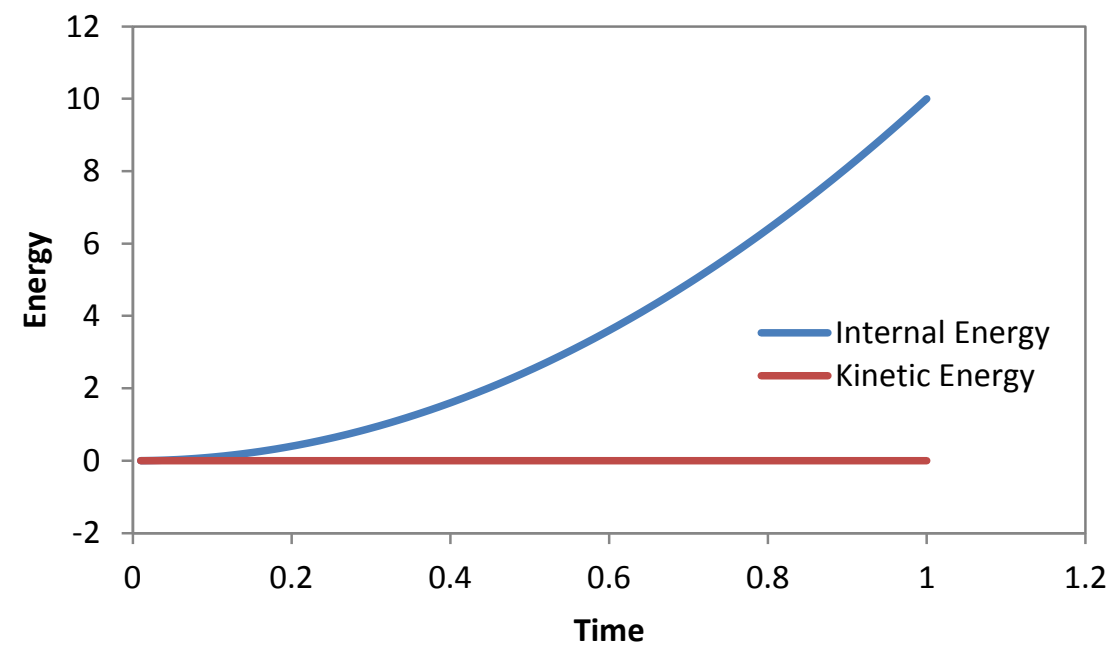

Figure 2-7 - Ideal quasi-static energy curve, with external work equal to internal energy and kinetic energy equal to zero. 


\section{CHAPTER 3}

\section{PRACTICAL EVALUATION OF THE HASHIN DAMAGE MODEL IN ABAQUS CAE}

A series of simple experiments were performed within Abaqus in order to evaluate the effects of the parameters within the Hashin damage model. The knowledge taken from this study would help in tuning the model to better match the physical results when more complex geometry is considered. In all, 6 different tension tests were performed in this study.

\subsection{Model Setup}

A quarter-symmetric tensile coupon model was developed in Abaqus CAE. The boundary conditions are shown below in Figure 3-1 and were chosen to replicate the conditions experienced in a uniaxial tensile test. The left edge constrains displacement in the 2 direction and rotation about the 1 and 3 axes. The bottom edge constrains displacement in the 1 direction and rotation about the 2 and 3 axes. The final boundary condition represents the applied load on the specimen.

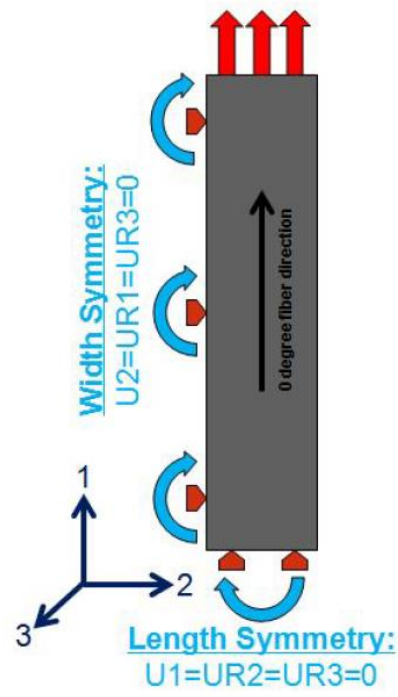

Figure 3-1 - Quarter symmetry tensile coupon with boundary conditions shown [18]. 
The load was applied by means of a reference point specified above the top edge of the specimen. Then, a kinematic coupling constraint was defined between the reference point and the nodes on the top edge of the tensile coupon. By doing this, the degrees of freedom of the top edge are tied to those of the reference point. A displacement boundary condition was then applied to the reference point (or velocity for the dynamic explicit models).

Table 3-1 and Table 3-2 below outline the material properties and dimensions used in the models respectively. These values were taken from a similar study performed at The Ohio State University [18].

Table 3-1 - Material properties for CFRP used in tensile FEA experiments.

\begin{tabular}{|c|c|c|c|}
\hline & Property & Units & Value \\
\hline & Density & tonne $/ \mathrm{mm}^{3}$ & 0.01 \\
\hline \multirow{6}{*}{ 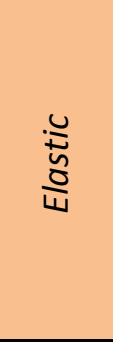 } & E1 & $\mathrm{MPa}$ & 123520 \\
\hline & E2 & $\mathrm{MPa}$ & 6516 \\
\hline & v12 & -- & 0.321 \\
\hline & G12 & $\mathrm{MPa}$ & 2494 \\
\hline & G13 & $\mathrm{MPa}$ & 2494 \\
\hline & G23 & $\mathrm{MPa}$ & 2300 \\
\hline \multirow{7}{*}{ 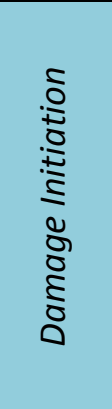 } & $\mathrm{X}^{\mathrm{t}}$ & $\mathrm{MPa}$ & 1429 \\
\hline & $X^{c}$ & $\mathrm{MPa}$ & 530 \\
\hline & $Y^{t}$ & $\mathrm{MPa}$ & 41 \\
\hline & $Y^{c}$ & $\mathrm{MPa}$ & 145 \\
\hline & $S^{\prime}$ & $\mathrm{MPa}$ & 83.4 \\
\hline & $S^{t}$ & $\mathrm{MPa}$ & 83.4 \\
\hline & $\alpha$ & -- & 0 \\
\hline \multirow{4}{*}{ 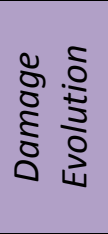 } & $\mathrm{G}^{\mathrm{lt}}$ & $\mathrm{mJ} / \mathrm{mm}^{2}$ & 12.5 \\
\hline & $\mathrm{G}^{\mathrm{lc}}$ & $\mathrm{mJ} / \mathrm{mm}^{2}$ & 12.5 \\
\hline & $\mathrm{G}^{\mathrm{tt}}$ & $\mathrm{mJ} / \mathrm{mm}^{2}$ & 1 \\
\hline & $G^{t c}$ & $\mathrm{~mJ} / \mathrm{mm}^{2}$ & 1 \\
\hline \multirow{4}{*}{ 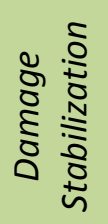 } & $\eta^{\text {It }}$ & -- & 0.001 \\
\hline & $\eta^{\text {lc }}$ & -- & 0.001 \\
\hline & $\eta^{t t}$ & -- & 0.005 \\
\hline & $\eta^{\mathrm{tc}}$ & -- & 0.005 \\
\hline
\end{tabular}


Table 3-2 - Dimensions of tensile specimen used in FEA experiments.

\begin{tabular}{|l|l|r|}
\hline Property & \multicolumn{1}{|c|}{ Units } & \multicolumn{1}{c|}{ Value } \\
\hline Length & $\mathrm{mm}$ & 69 \\
\hline Width & $\mathrm{mm}$ & 12.54 \\
\hline$t_{\text {ply }}$ & $\mathrm{mm}$ & 0.24 \\
\hline Layup & -- & {$\left[0_{6}\right]^{*}$} \\
\hline \multicolumn{3}{|c}{ *initial }
\end{tabular}

\subsection{Implicit vs. Explicit 0 Degree Tensile Test}

The first test performed was a zero degree tensile test to evaluate the Hashin damage model using both an implicit and explicit solver. The element size was altered from a $5 \mathrm{~mm}$ seed size to a $0.5 \mathrm{~mm}$ seed size. This was to investigate solution convergence and mesh size dependency for the Hashin damage model. The reaction force was tracked for the reference point as well as the displacement. Figure 3-2 shows the results from the Abaqus/Standard solver, Figure 3-3 shows the results from the Abaqus/Explicit, and Figure 3-4 compares the two solutions. 


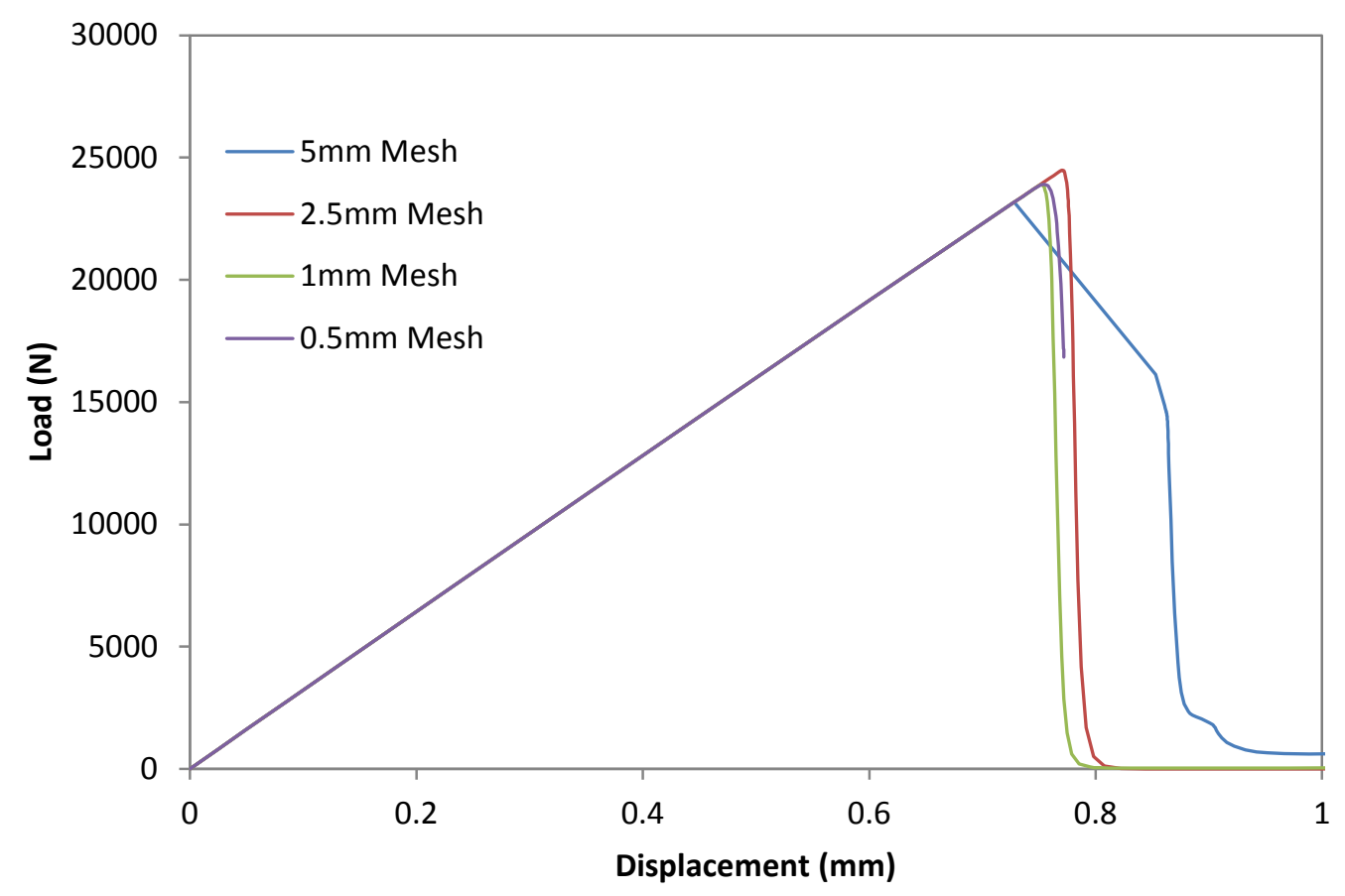

Figure 3-2 - Abaqus/Standard results for CFRP tensile specimen using various mesh sizes.

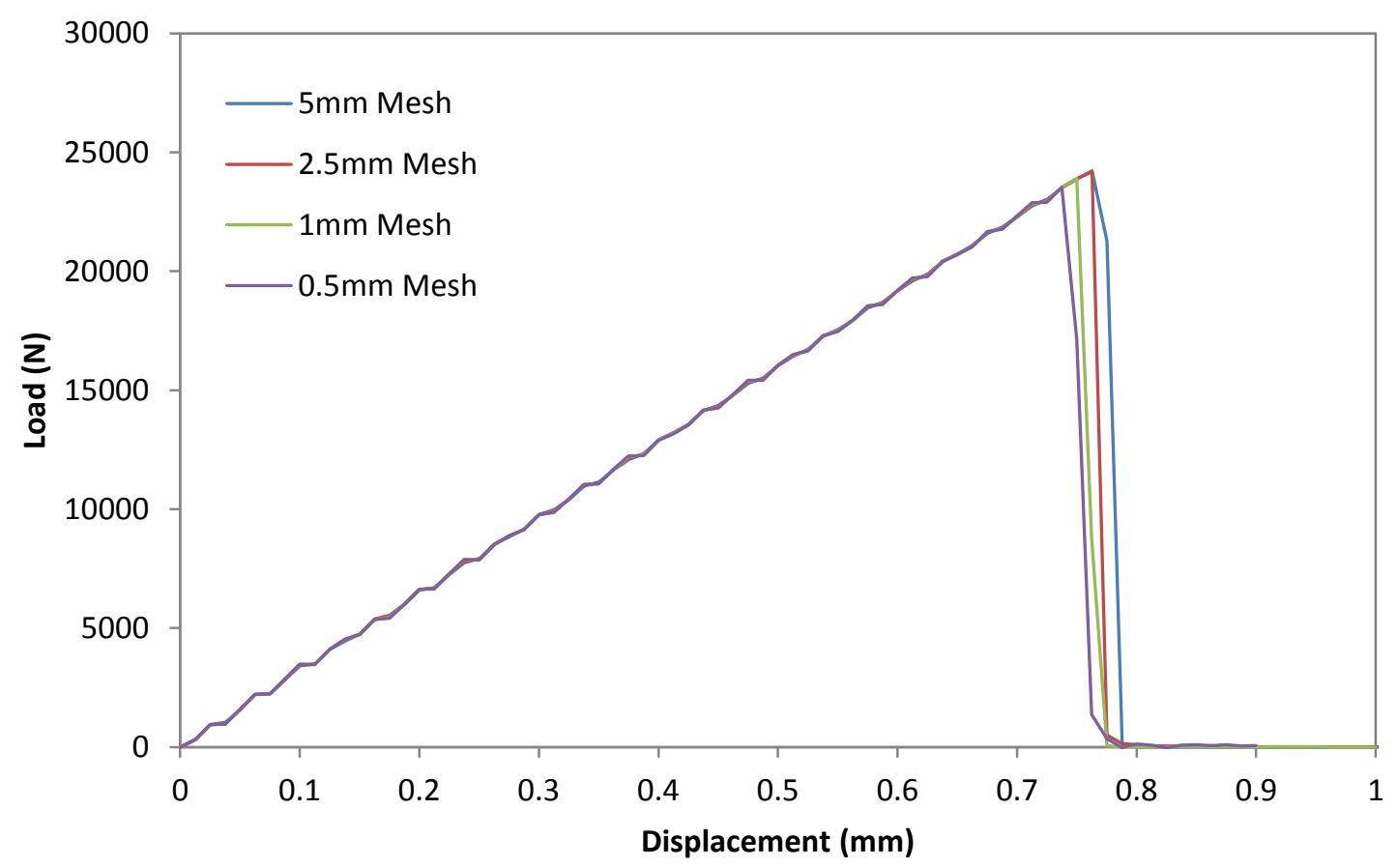

Figure 3-3 - Abaqus/Explicit results for CFRP tensile specimen using various mesh sizes. 
Both the implicit and explicit solvers capture the basic behavior of CFRP damage. The material exhibits linear elastic behavior up until failure. When the failure load is reached, all of the material stiffness is lost and the load drops to essentially zero. The solutions, however, do show some mesh dependency. As shown in Figure 3-2, the mesh is very important for the implicit solution. A coarse mesh of $5 \mathrm{~mm}$ seeds did not produce realistic results. When the mesh seed size was decreased by half, however, the solution became more reasonable. For the explicit solver, the solution showed very little mesh dependency, as shown in Figure 3-3.

It should be noted that the explicit solver is a dynamic solver. This is why the load exhibits some fluctuations during the linear elastic phase. This is due to the stress wave that is propagating through the material. It should also be noted that the $0.5 \mathrm{~mm}$ mesh size implicit run "errored-out" after reaching the elastic limit.

Figure 3-4 below shows the mesh dependency results from the tests mentioned above. It can be concluded from these results that a mesh seed size of $1 \mathrm{~mm}$ provides a converged solution without introducing an excessive amount of degrees of freedom. Additionally, there is essentially no difference between the implicit and explicit solvers for this problem. Thus, this reaffirms the choice for using an explicit solver and will be further explored. 


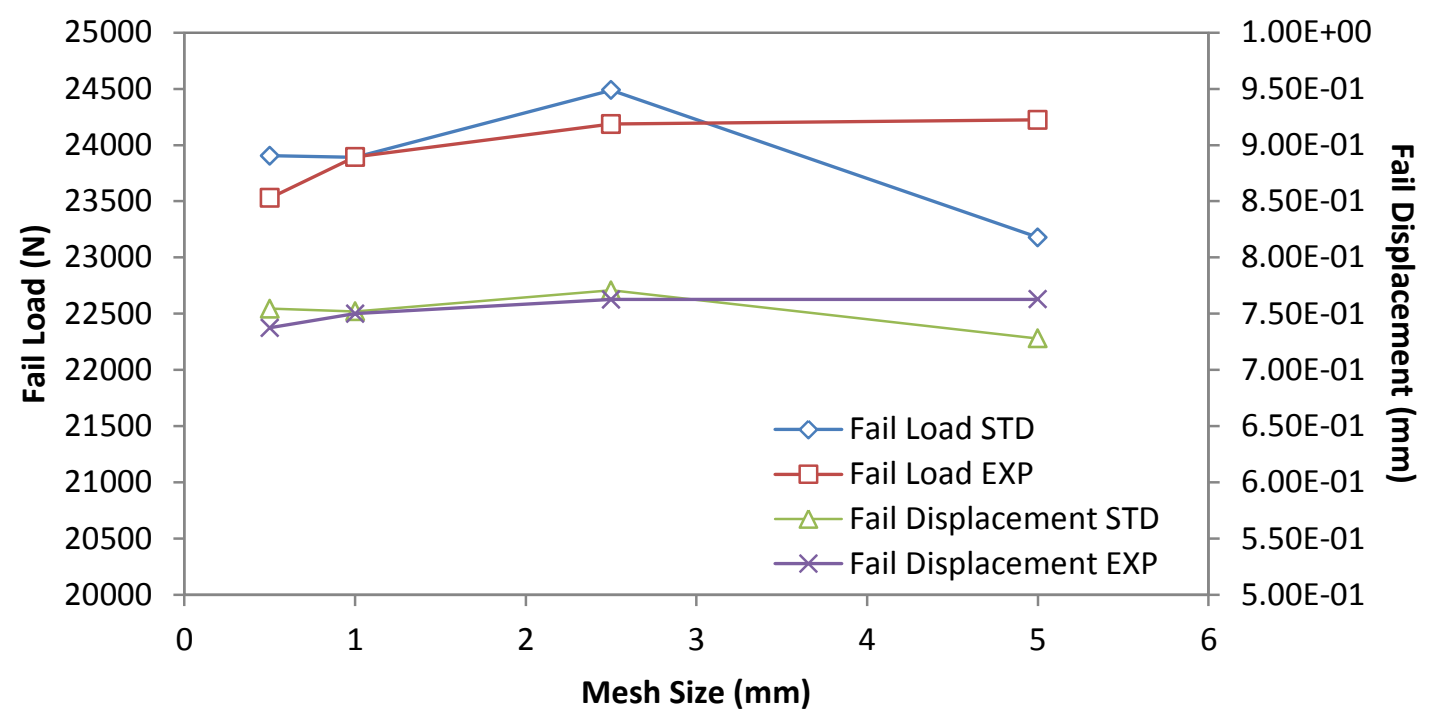

Figure 3-4 - Mesh size dependency results for Hashin damage model using a $\left[0_{6}\right]$ tensile speciemen.

\subsection{Mass Scaling and Smooth Step Amplitude Curve Application}

As mentioned above, in order to speed up the computation a fixed mass scaling was applied to the model in the form of scaling the density by a factor $f$. A series of Abaqus models were run using various values of $f$ ranging from $10^{6}$ to $10^{12}$. Figure 3-5 below shows the results from the test.

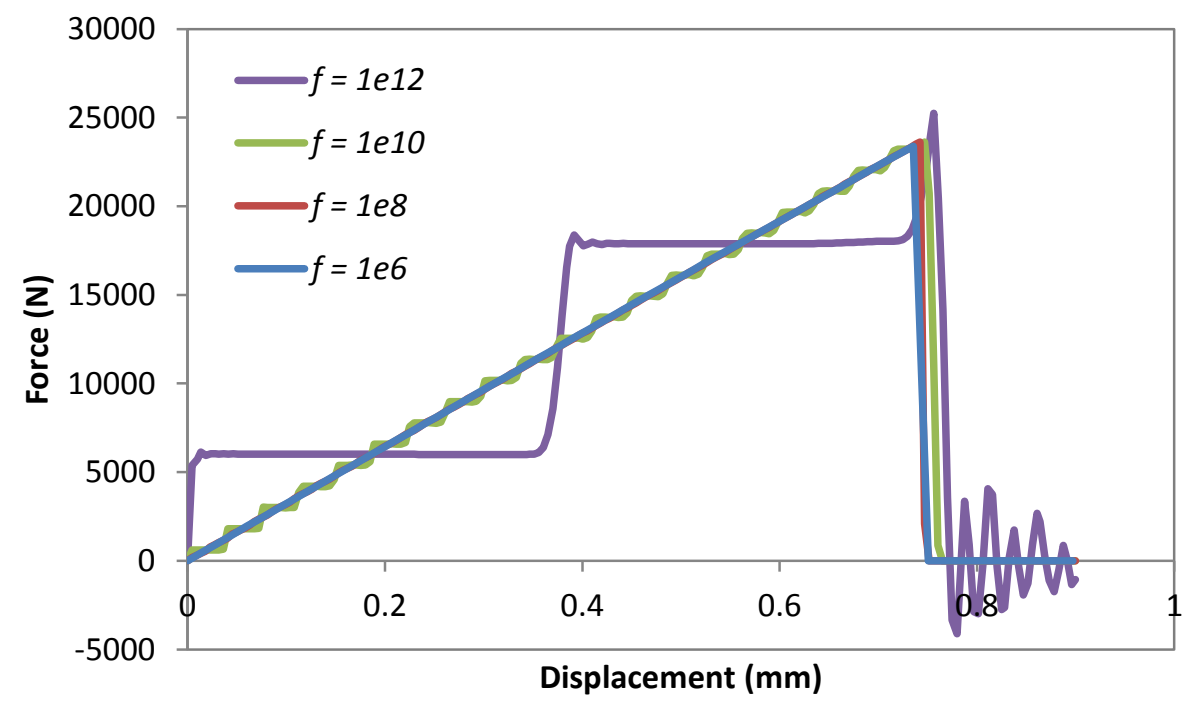

Figure 3-5 - Load versus displacement plot for 0 degree tensile specimen using various mass scaling factors. 
Examining the Figure 3-5 above, it is clear that the $10^{6}$ and $10^{8}$ mass scaling factors produce valid results. The $10^{10}$ factor seems close, but vibrations on the linear elastic portion produce undesirable results. Before coming to a conclusion as to what mass scaling factor should be utilized, it should be noted that it is most desirable to use the highest mass scaling factor as possible, as this reduces the solution time by a factor of $\sqrt{f}$. Figure 3-6 below shows the time to solution for the four mass scaling factors presented above. If the $10^{10}$ factor were to be used over the $10^{8}$ factor, Abaqus arrives at the solution nearly ten times faster.

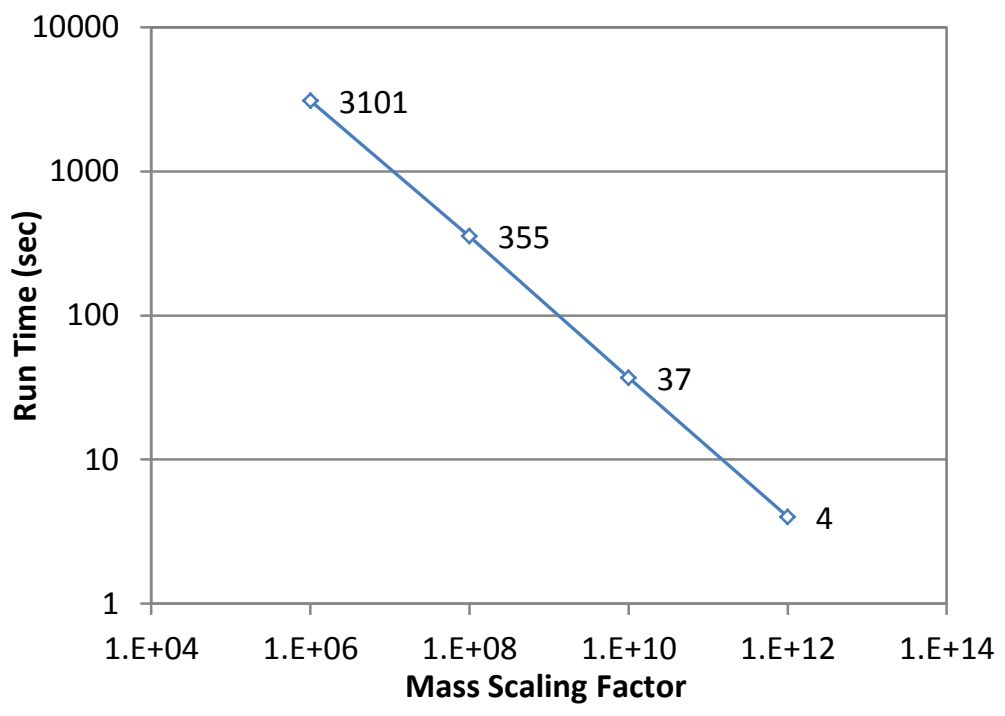

Figure 3-6 - Time to solution for various mass scaling factors.

There are measures that can be implemented to mitigate the vibrations caused by the stress wave and utilize the higher mass scaling factor. They will be discussed later in the section. First, it needs to be determined if the $10^{10}$ mass scaling factor is acceptable. For this, an energy balance needs to be considered. Essentially, the kinetic energy of the system needs to be a very small fraction of the internal energy. Figure 3-7 below shows the energy balance of the $10^{10}$ mass scaling factor run. Note that the kinetic energy is essentially zero up until failure. At failure, there are two separate bodies of the undergoing deformation or displacements. Since there is no material to resist motion, the body essentially experiences rigid body motion. This is why the 
kinetic energy spikes at failure. Some internal energy is present after failure possibly due to remnants of the degraded stiffness matrix continuing to resist some motion. It is unclear why this is the case and may be a topic for future work. However, since the kinetic energy is small up until failure, the mass scaling factor of $10^{10}$ is acceptable and will be utilized for subsequent studies.

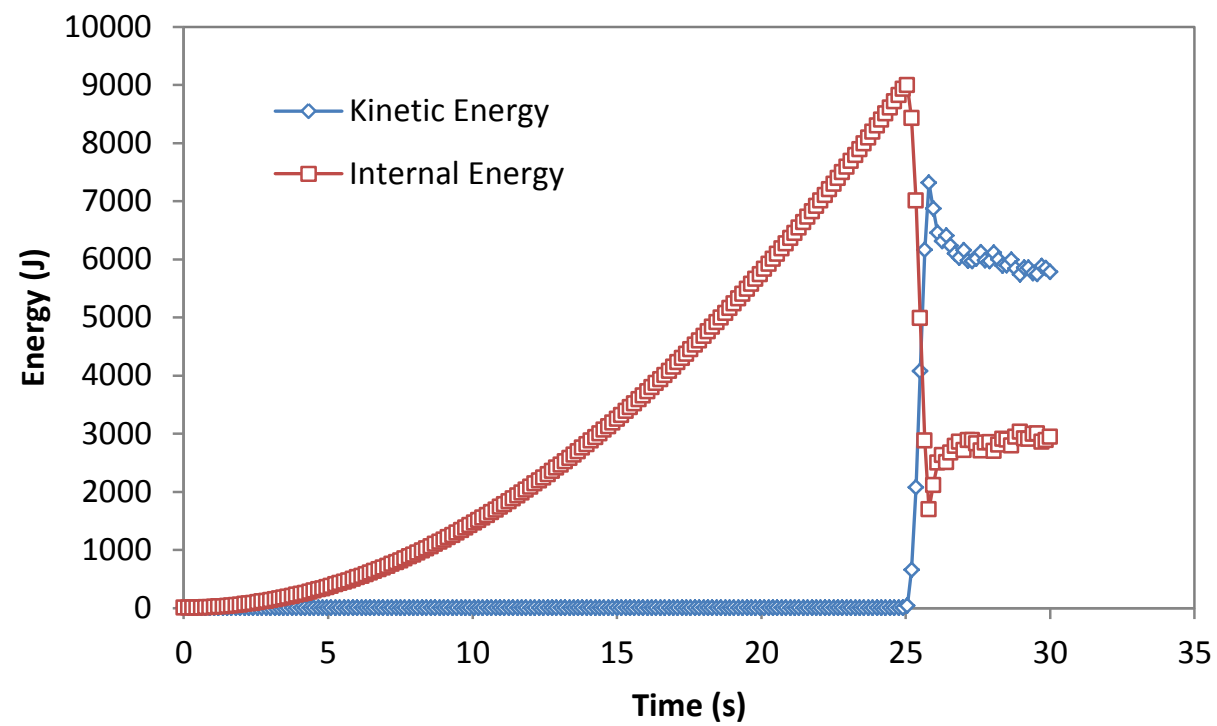

Figure 3-7 - Kinetic energy versus internal energy for 0 degree tensile specimen using a mass scaling factor of $10^{10}$.

After a mass scaling value of $f=10^{10}$ was determined, a smooth step amplitude curve was applied to the velocity boundary condition in an effort to get rid of the vibrations caused by the stress wave due to the immediate application of load on the specimen. Various step times were used to determine an appropriate value. Note that the total time of the job was 100 seconds. Figure 3-8 below outlines the results of the study. 


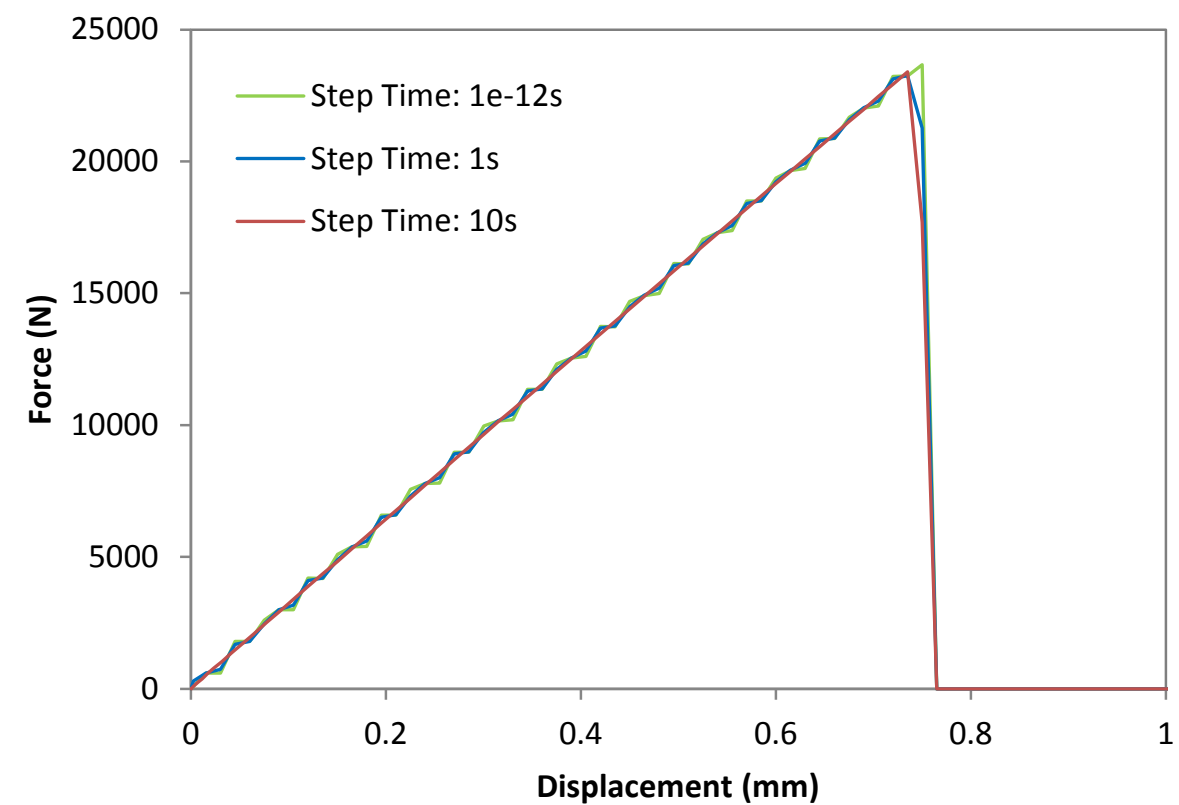

Figure 3-8 - Load versus displacement for 0 degree tensile specimen utilizing different smooth step amplitude times.

By using a step time of 10 seconds for the smooth step amplitude, the vibrations caused by the stress wave were completely mitigated. This demonstrates that using the smooth step amplitude to slowly apply load to a structure with very high mass scaling factors can be utilized and thus a more practical solution can be obtained.

\subsection{Fracture Energy}

Next, the effects of fracture energy within the Hashin damage model were investigated. The fracture energy $G_{c}$ is defined as the area under the equivalent stress versus equivalent displacement curve as shown previously in section 2.3.2 Damage Evolution. This is essentially the area under the stress-strain curve. The fracture energy for fiber failure was varied from 1 $\mathrm{mJ} / \mathrm{mm}^{2}$ to $1000 \mathrm{~mJ} / \mathrm{mm}^{2}$ by factors of ten in the zero degree tensile specimen. The matrix failure fracture energy was held constant at $1 \mathrm{~mJ} / \mathrm{mm}^{2}$. The results of the study are shown below in Figure 3-9. 


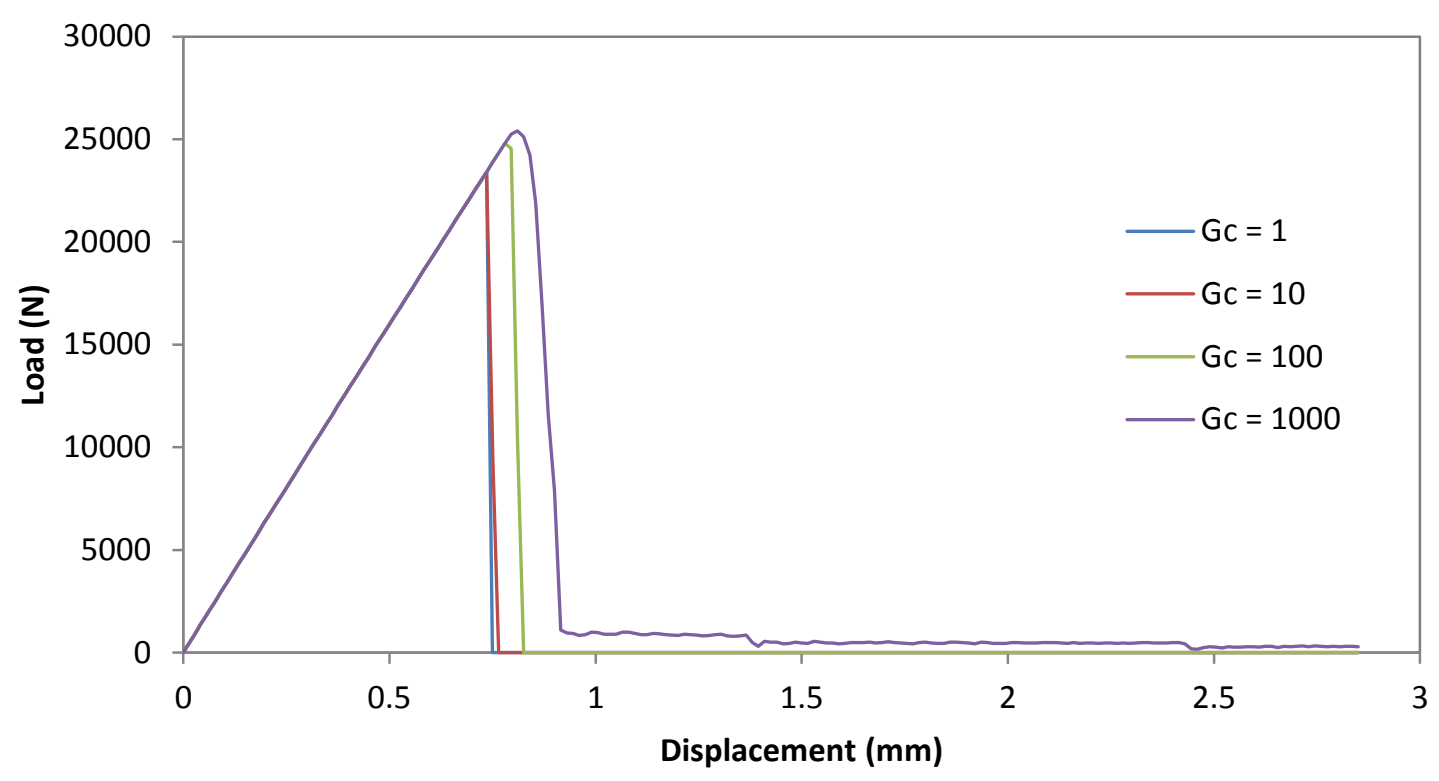

Figure 3-9 - Load versus displacement for 0 degree tensile specimen utilizing different fiber fracture energies.

As shown above, the fracture energy affects the failure response of the material significantly. The response between the $1 \mathrm{~mJ} / \mathrm{mm}^{2}$ and $10 \mathrm{~mJ} / \mathrm{mm}^{2}$ trials are nearly identical, with the later trial building up slightly more load than the former. When the fracture energy was set to $100 \mathrm{~mJ} / \mathrm{mm}^{2}$ (or ten times that of the baseline value) the response is significantly different. The load builds up to a maximum value of $25 \mathrm{kN}$ and then catastrophically fails. When the fracture energy was set to $1000 \mathrm{~mJ} / \mathrm{mm}^{2}$, the response did not reflect the response of a zero degree tensile specimen. The load maximizes at a value of approximately $25 \mathrm{kN}$ and tapers off relatively slowly at first. Then, the load dramatically decreases to a value of approximately $1000 \mathrm{~N}$ and slowly decreases as the specimen continues to be pulled. The rounded peak load and non-zero force after failure is undesirable and does not represent the response of a zero degree tensile specimen.

From this study, it can be concluded that the fracture energy does have a significant effect on the failure response of the material. It appears that too little fracture energy doesn't affect the response significantly, however too much fracture energy gives undesirable results. 


\subsection{Maximum Degradation}

Next, the maximum degradation value was altered to see the effects of it within the Hashin damage model. The maximum degradation value was set to values of 1, 0.8, 0.6, 0.4 and 0.2. Note that the default value for the maximum degradation variable is 1 . Figure 3-10 below shows the results from the study.

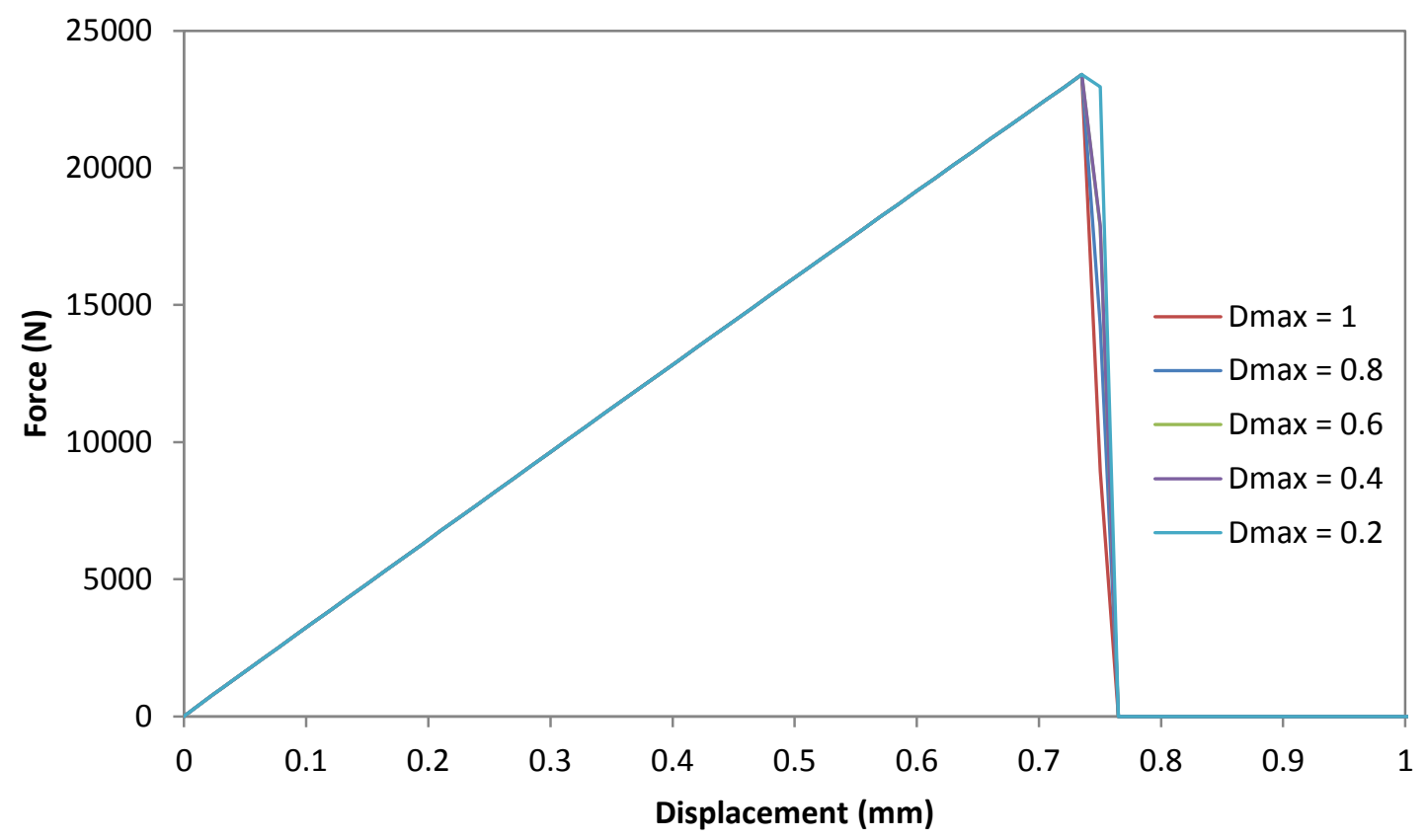

Figure 3-10 - Force versus deflection curve for 0 degree tensile specimen with varying maximum degradation values.

As shown above, there are no major changes to the response of the material. With a $D_{\max }$ value of 0.2 , the onset of failure appears to be slightly less sudden compared to the other values. Additionally, the damaged portion of the force deflection curve is slightly different for each value of $\mathrm{D}_{\max }$. Note that the $\mathrm{D}_{\max }=0.6$ and $\mathrm{D}_{\max }=0.4$ curves are almost identical. Interestingly, all of the curves reach zero load at the same displacement of approximately $0.765 \mathrm{~mm}$. Figure 3-11 below shows a detailed view of the damage region. As shown below, the $\mathrm{D}_{\max }$ variable affects the degraded stiffness of the part. The higher the value of the $D_{\max }$ variable, the more the stiffness is degraded. 


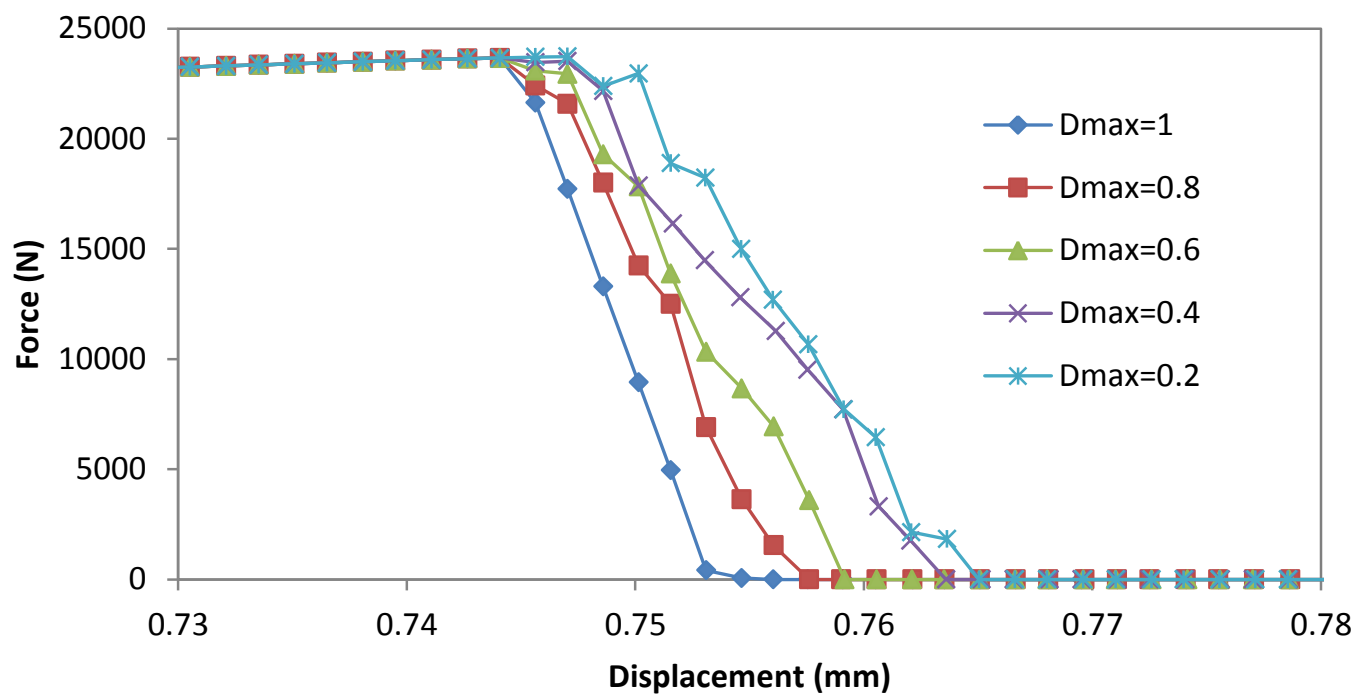

Figure 3-11 - Detailed view of damaged portion of load versus displacement response of 0 degree tensile specimen.

While the material response is relatively unchanged for varying values of $D_{\max }$, the visualization of the damaged specimens are quite different. Figure 3-12 below shows the damaged tensile specimen at the onset of damage. As shown, as the $\mathrm{D}_{\max }$ value is reduced, the number of elements that are removed from the visualizer increases. This is because the $D_{\max }$ variable affects the STATUS variable. When the degradation variable in an element reaches the value of $D_{\max }$, the STATUS variable switches from 1 to 0 . This deactivates the element and removes it from the visualizer. 


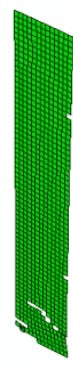

(a)

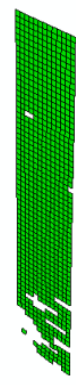

(b)

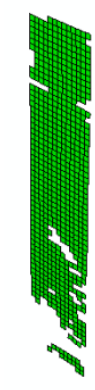

(c)

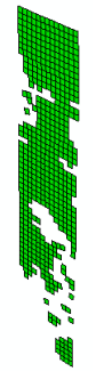

(d)

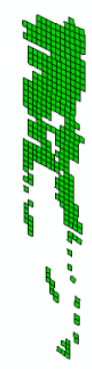

(e)

Figure 3-12 - Deformed shaped of 0 degree tensile specimen for $D_{\max }$ value of (a) 1 , (b) 0.8 , (c) 0.6, (d) 0.4 , and (e) 0.2 .

While it is clear that the value of $D_{\max }$ changes the visualization of part, it is still unclear whether it changes the material response significantly. The tensile test provides a rapid onset of damage to the fiber composite, meaning nearly all of the elements at the failure location will fail at the same time. A different test, such as a three point bend test, may be a better option for evaluating the effects of the $D_{\max }$ variable. 


\section{CHAPTER 4}

\section{AXIAL TUBE CRUSH TESTING AND FEA}

After investigating the parameters within the Hashin damage model in Abaqus, various tube specimens were crushed in the lab in order to validate the model and method developed by an earlier thesis [14]. A tube constructed of carbon fiber was sectioned and tested and the physical results were compared with the FEA results

\subsection{Axial Tube Crush Testing}

A CFRP tube was procured and consisted of two layers of T300/RS-3C cloth and 14 layers of M55J/RS-3C unidirectional tape. Table 4-1 below outlines the tube construction. The tube was sectioned into 3 inch lengths and a 51 degree bevel was incorporated into the leading edge of the tube.

Table 4-1 - CFRP tube specifications.

\begin{tabular}{|l|l|r|}
\hline \multicolumn{1}{|c|}{ Property } & Units & \multicolumn{1}{c|}{ Value } \\
\hline Length & in & 3 \\
\hline Inner Diameter & in & 0.005 \\
\hline$t_{\text {ply }} T 300$ & in & 0.004 \\
\hline$t_{\text {ply }}$ M55J & in & {$\left[45_{c} / 0_{14} / 45_{c}\right]$} \\
\hline Layup & -- &
\end{tabular}

A test fixture was constructed to support the tube during the axial crush. The fixture was a weldment consisting of a grip tab, base, support ring, and shim to allow for future testing of different sized tubes. Figure 4-1 below shows a CAD drawing of the fixture used for testing. 


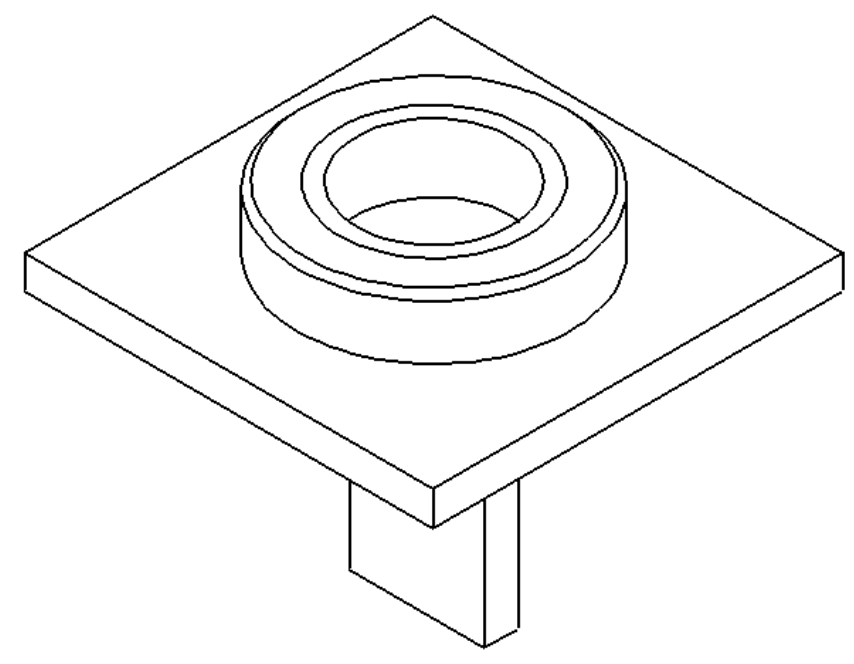

Figure 4-1 - CAD drawing of tube crush test fixture.

The test fixture was loaded into the bottom grip of the Instron test machine and was adjusted until it was level. Next, a tube was loaded into the fixture with the bevel end facing up. Finally, the impactor plate was loaded into the top grip of the Instron and adjusted until it sat level. The tube and fixture were then raised until the top of the tube was just below the impactor plate. The Instron was then programmed to crush the tube using displacement control at a rate of $0.001 \mathrm{in} / \mathrm{s}$. Figure 4-2 and Figure 4-3 below show the results of the test. 


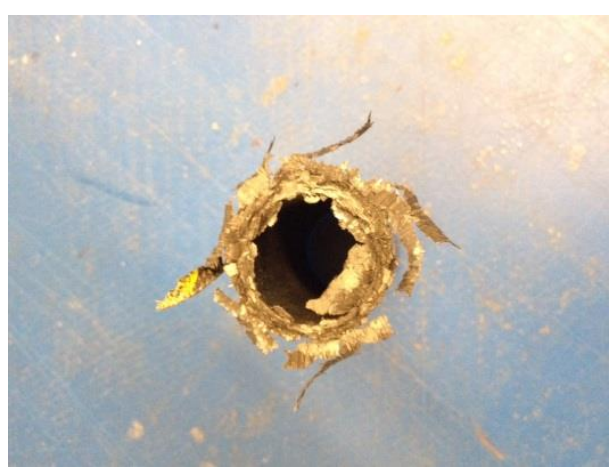

(a)

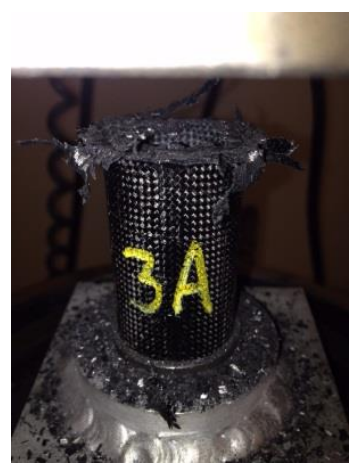

(c)

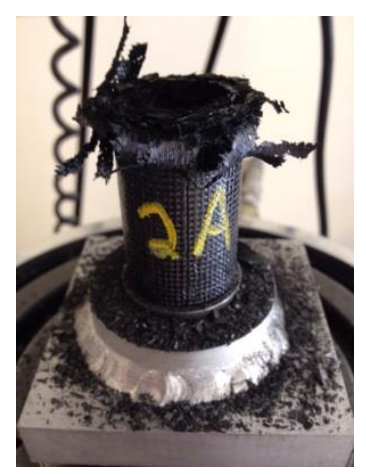

(b)

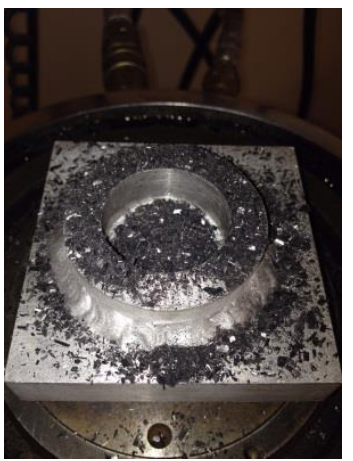

(d)

Figure 4-2 - Post crush of specimen (a) 1A, (b) 2A, and (c) 3A. (d) Typical debris after a crush test.

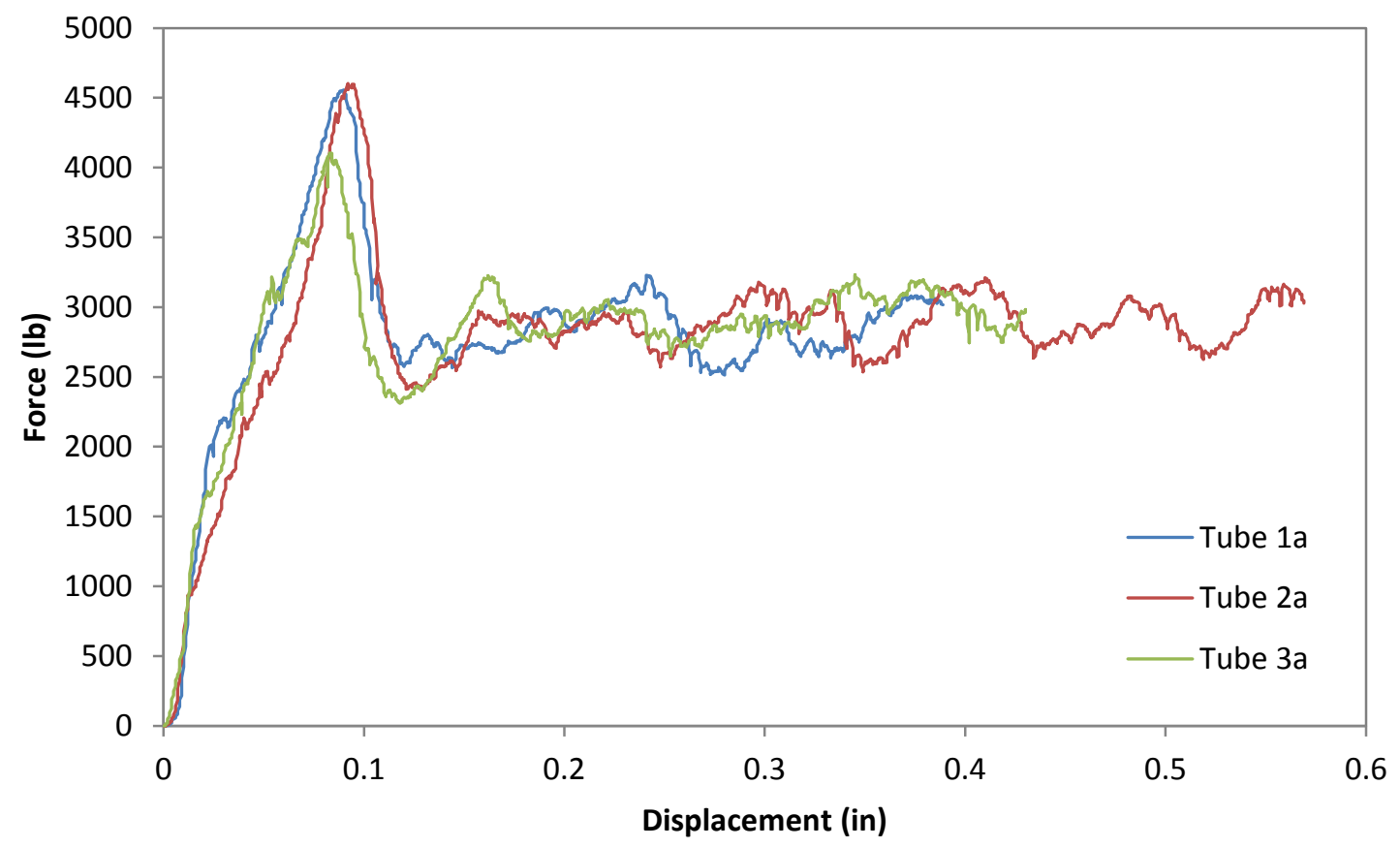

Figure 4-3 - Load versus displacement data from tube axial crush tests. 
All of the tubes exhibit essentially the same behavior. Each tube builds up load until it peaks at an ultimate failure point. Then the load drops and a steady crush ensues. While it is useful to analyze the force versus displacement plot, it is more convenient to analyze the performance in terms of specific crushing stress (SCS) [14]. SCS is determined using (4-1) below where $P$ is the applied load, $A$ is the cross sectional area, and $\rho$ is the specimen's density. Essentially, it is the compressive stress divided by the density.

$$
S C S=\frac{P}{A \rho}
$$

Another metric to evaluate impact absorbing devices is specific energy absorption (SEA). This is determined using (4-2) below.

$$
S E A=S C S_{\text {mean }}=\frac{P_{\text {mean }}}{A \rho}
$$

Finally, the crush load efficiency (CLE) is defined using (4-3) below.

$$
C L E=\frac{P_{\text {mean }}}{P_{\text {peak }}}=\frac{P_{\text {mean }} / \rho A}{P_{\text {peak }} / \rho A}=\frac{S E A}{S C S_{\text {peak }}}
$$

Using the metrics defined above, the SCS versus displacement plot is shown below in Figure 4-4. Additionally, the key performance parameters are outlined in Table 4-2 below. 


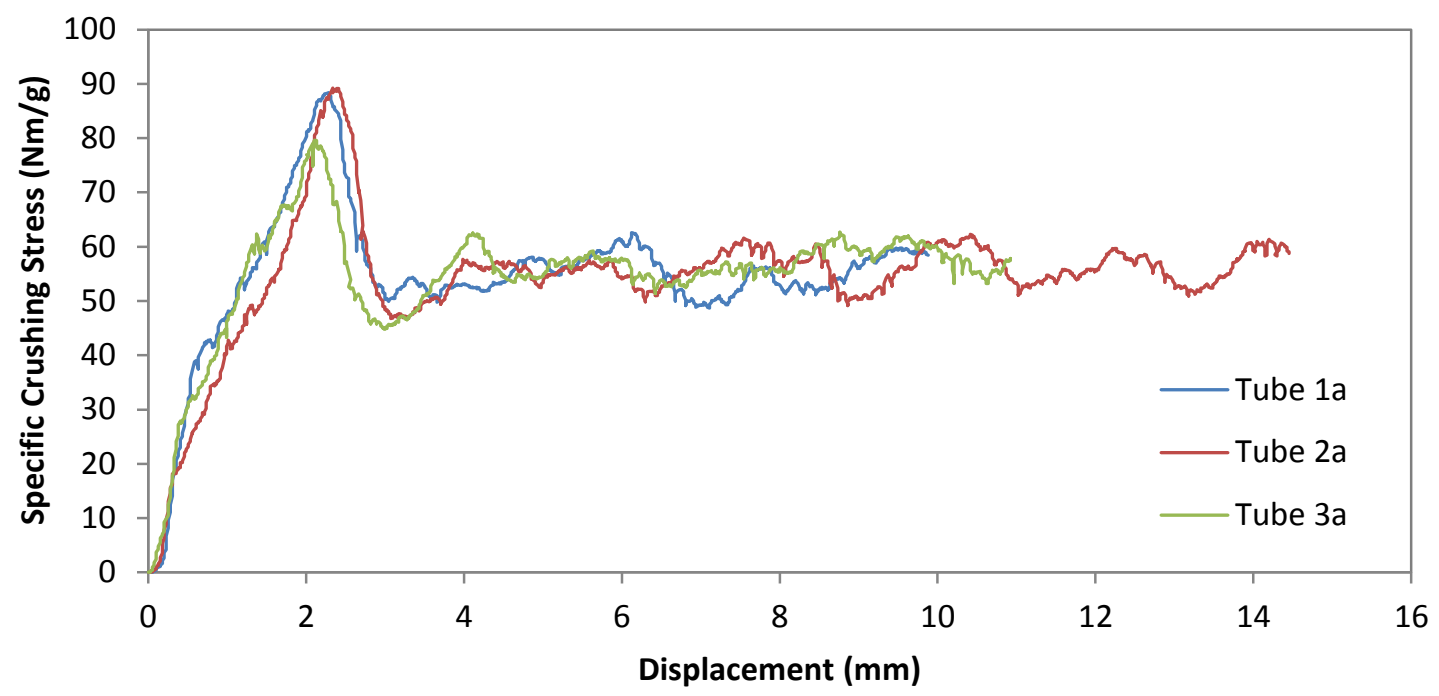

Figure 4-4 - Specific crushing stress versus displacement for axial crush test of CFRP tubes.

Table 4-2 - Crush performance characteristics of CFRP tubes.

\begin{tabular}{|l|r|r|r|}
\hline Specimen & SCS Peak [Nm/g] & SEA [J/g] & CLE [\%] \\
\hline 1a & 88.34 & 54.37 & $61.55 \%$ \\
\hline 2a & 89.19 & 54.25 & $60.82 \%$ \\
\hline 3a & 79.59 & 54.28 & $68.20 \%$ \\
\hline Average & $\mathbf{8 5 . 7 1}$ & $\mathbf{5 4 . 3 0}$ & $\mathbf{6 3 . 5 2 \%}$ \\
\hline
\end{tabular}

The values above were used as the target for the following finite element analysis. The goal was to reasonably replicate the physical test using the built in Hashin Damage model for fiber reinforced composites.

\subsection{FEA Model Development}

The finite element model was constructed in Abaqus as an explicit dynamic nonlinear analysis. The model was based on the two layer tube model developed by Roberts [14]. This was done to try and capture the two fronds that developed during the test. For ease of model definition, the US Customary unit system was used. The model started by extruding two circles of diameter 1.0333 in and 1.1549 in representing the mid-plane of half of the layup. The tube was 
placed under a rigid impactor plate. The base of the tube was constrained in all three translational degrees of freedom and the rotational degrees of freedom about the transverse axes. The longitudinal axis rotational degree of freedom was released. This was done to emulate the boundary conditions of the test fixture. The impactor was allowed to move in the tube's axial direction, but was constrained in all other degrees of freedom.

Next, the material properties were defined. As mentioned above, the tubes were constructed with T300/RS-3C cloth and M55J/RS-3C unidirectional tape. The actual material properties cannot be listed since they are proprietary to the manufacturer. Tables Table 4-3 and Table 4-4 below outline typical material properties of M55J/RS-3C and T300/RS-3C respectively [19]. 
Table 4-3 - Material properties for M55J/RS-3C unidirectional tape CFRP.

\begin{tabular}{|c|c|c|c|}
\hline & Property & Units & Value \\
\hline & Density & Lbf*s ${ }^{2} /$ in $^{4}$ & $1.501 \mathrm{E}-04$ \\
\hline \multirow{6}{*}{ 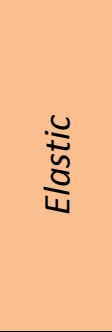 } & E1 & Msi & 46.2 \\
\hline & E2 & Msi & 0.82 \\
\hline & v12 & -- & 0.33 \\
\hline & G12 & Msi & 0.13 \\
\hline & G13 & Msi & 0.13 \\
\hline & G23 & Msi & 0.13 \\
\hline \multirow{7}{*}{ 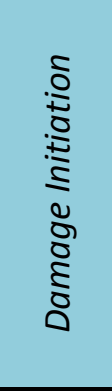 } & $\mathrm{Xt}$ & ksi & 290 \\
\hline & $X c$ & ksi & 129 \\
\hline & $Y t$ & ksi & 4 \\
\hline & Yc & ksi & 14.9 \\
\hline & SI & ksi & 10.9 \\
\hline & St & ksi & 10.9 \\
\hline & $\alpha$ & -- & 0 \\
\hline \multirow{4}{*}{ 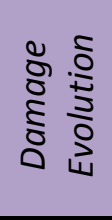 } & Glt & lbf/in & $2.264 \mathrm{E}+06$ \\
\hline & Glc & lbf/in & $6.392 \mathrm{E}+05$ \\
\hline & Gtt & $\mathrm{lbf} /$ in & $1.611 \mathrm{E}+05$ \\
\hline & Gtc & Ibf/in & $2.706 \mathrm{E}+06$ \\
\hline \multirow{4}{*}{ 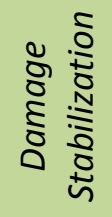 } & $\eta \mid t$ & -- & N/A \\
\hline & nlc & -- & N/A \\
\hline & $\eta t t$ & -- & N/A \\
\hline & ntc & -- & N/A \\
\hline
\end{tabular}


Table 4-4 - Material properties used of T300/RS-3C cloth CFRP.

\begin{tabular}{|c|c|c|c|}
\hline & Property & Units & Value \\
\hline & Density & Lbf*s ${ }^{2} /$ in $^{4}$ & $1.449 \mathrm{E}-4$ \\
\hline \multirow{6}{*}{$\begin{array}{l}\stackrel{U}{\tilde{w}} \\
\frac{o}{w}\end{array}$} & E1 & Msi & 9.7 \\
\hline & E2 & Msi & 9.7 \\
\hline & v12 & -- & 0.05 \\
\hline & G12 & Msi & 0.70 \\
\hline & G13 & Msi & 0.70 \\
\hline & G23 & Msi & 0.70 \\
\hline \multirow{7}{*}{ 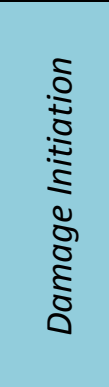 } & $X t$ & ksi & 121 \\
\hline & $X_{c}$ & Ksi & 118 \\
\hline & $\mathrm{Yt}$ & ksi & 121 \\
\hline & $Y_{c}$ & ksi & 118 \\
\hline & $\mathrm{SI}$ & ksi & 11 \\
\hline & St & ksi & 11 \\
\hline & $\alpha$ & -- & 0 \\
\hline \multirow{4}{*}{ 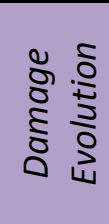 } & Glt & $\mathrm{lbf} /$ in & $2.264 E+06$ \\
\hline & Glc & $\mathrm{lbf} /$ in & $6.392 E+05$ \\
\hline & Gtt & $\mathrm{lbf} / \mathrm{in}$ & $2.264 E+06$ \\
\hline & Gtc & $\mathrm{lbf} /$ in & $6.392 E+05$ \\
\hline \multirow{4}{*}{ 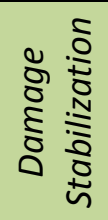 } & $\eta \mathrm{lt}$ & -- & N/A \\
\hline & nlc & -- & $\mathrm{N} / \mathrm{A}$ \\
\hline & $\eta t t$ & -- & N/A \\
\hline & ntc & -- & N/A \\
\hline
\end{tabular}

Four composite sections were then defined: inner trigger, outer trigger, inner tube, outer tube. The layups used for each are defined below in Table 4-5. Each layer had only one section point reduced from three to reduce the computational cost of the analysis [14]. The material orientation was based on a discrete field definition. The surface of the tube was selected for the normal axis definition. Now, the 3 axis or out-of-plane axis of the composite material will always be normal to the surface of the tube. Then the primary axis was defined as a vector that was parallel to the tube's longitudinal axis, in this case $<0,0,1>$. This would set the default orientation of the fibers in the tube's axial direction. 
Table 4-5 - Layup definitions for various sections of the tube.

\begin{tabular}{|c|c|}
\hline Region & Layup \\
\hline Inner Trigger & {$\left[45_{c} / 0_{3}\right]$} \\
\hline Outer Trigger & {$\left[0_{3} / 45_{c}\right]$} \\
\hline Inner Tube & {$\left[45_{c} / 0_{7}\right]$} \\
\hline Outer Tube & {$\left[0_{7} / 45_{c}\right]$} \\
\hline
\end{tabular}

Next the parts were meshed. The tubes were partitioned into three sections: trigger, upper tube, and main tube. The trigger was sectioned as the top 0.042 in of the tube and angled 51 degrees inward to represent the beveled edge on the physical tube. Figure 4-5 shows the cross section of the mesh at the trigger. This is a similar method that Huang and Wang [20] used to model the trigger on their tube. The upper tube incorporated the next 0.120 in of the tube and the main tube was the remaining section of the tube. The outer and inner tubes had the same partitioning scheme.

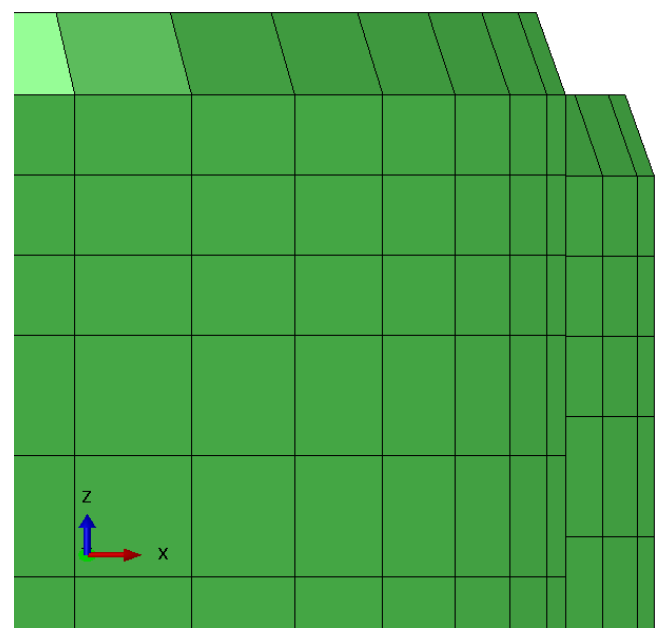

Figure 4-5 - Trigger portion of two layer tube FEM. 
The tubes were seeded with 0.060 in global seed size. This would make the elements approximately 0.060 in $x 0.060$ in in size. The trigger and upper tube partition were set to have fully integrated linear quadrilateral (S4) elements while the main tube was set to have reduced integrated linear quadrilateral (S4R) elements. This was done to reduce the computational cost of the analysis. The S4R elements had a pure stiffness based hourglass control to prevent hourglassing [8] with all scaling factors set to a value of 1 . All elements had a maximum degradation defined as 0.95 . This would guarantee that elements would be deleted once significantly damaged. All sections were set for element deletion. In all, the mesh had 5824 nodes, 448 S4, and 5264 S4R elements. Figure 4-6 below shows the mesh with the plate removed.

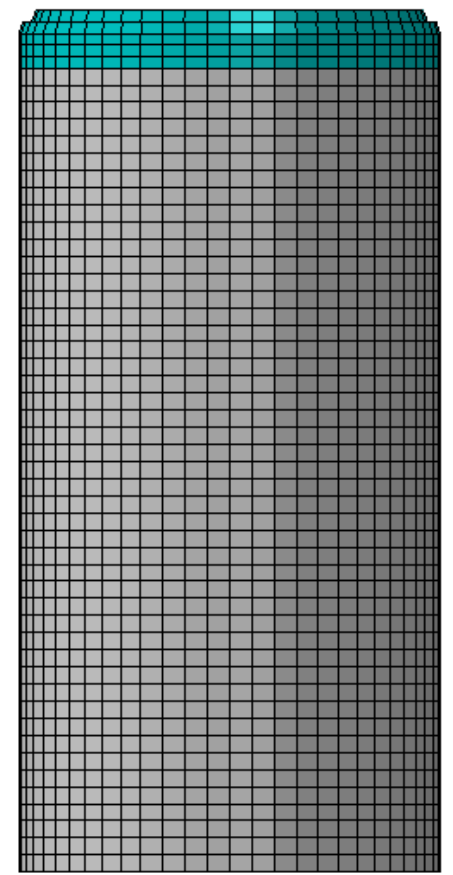

Figure 4-6 - Overview of two layer tube FEM with plate instance removed. Turquoise section represents fully integrated elements; grey section represents reduced integration elements.

Once assembled, the general contact algorithm was defined. The general contact algorithm allows the user to define contact for many or all regions of a model with a single interaction. It uses a single contact domain rather than contact pairs and is robust for explicit type analyses. A global contact interaction property was defined to control the behavior of the contact. 
A tangential friction behavior was defined using frictionless friction formulation. Finally, a "hard" contact pressure-overclosure was specified.

An impact contact definition was made for the interaction between the impactor and the tubes. This used a penalty tangential friction formulation with a coefficient of 0.2 . A damping coefficient of 0.2 was applied in an effort to smooth out the response. Finally, a "hard" contact pressure-overclosure was defined that allowed separation after contact. This must be done when using contact dampening.

\subsection{Modeling Issues Encountered}

Early on, the most encountered error was excessive element distortions and/or rotations. For excessive distortions, essentially the element has become so distorted that its area or volume has become mathematically negative. Similarly, excessive rotations occur when the incremental rotation of a given node exceeds a limit defined by Abaqus. These errors can onset suddenly when the analysis seems to be running fine. Usually, it can be traced back to a contact related problem, however in the case of this analysis, it was found to be related to an issue with offset elements.

In composite FEA it is often convenient to define geometry that is not at the mid-plane of the material. For example, the analyst might choose to use the tool surface as the definition of the geometry and define the layups from that surface. This causes the nodal plane to be offset from the mid-plane of the element. By having that offset there, an error is introduced on the order of the offset distance squared. While this is fine for small displacement analyses, an analysis such as this deals with extremely large deformations. This introduces a significant amount of error as it is integrated over each time step.

The original tube model used the tool surface as the reference surface. This was corrected such that the nodal plane coincided with the midplane of the tube. Although the geometry did not 
perfectly match that of the test article, the approximation was close enough to give credible results. Additionally, errors related to excessive distortions and rotations were eliminated.

Long run times were also encountered early on. In order to mitigate this, Abaqus's "semiautomatic" mass scaling was introduced. Rather than applying a blanket scaling factor to the density of the material, Abaqus checks the stable time increment of each element at set increments [17]. If the stable time increment is less than the target time increment, the mass of that particular element is scaled appropriately. This way, small elements don't effect the run time of the model as severely. Additionally, elements that are severely deformed don't reduce the stable time increment of the model such that run times become unreasonable.

\subsection{Finite Element Model Results and Discussion}

The finite element model was run on the Cal Poly Mechanical Engineering server using Abaqus v13-2. The server uses two Intel Xenon $2.80 \mathrm{GHz}$ processors with $16 \mathrm{~GB}$ of RAM operating in Windows Server 2008 R2 (64 bit). The model utilized parallelization such that it was solved in two domains. Additionally, full nodal precision was used to increase accuracy. The model took approximately 3 hours to run.

The first check done was to make sure the ratio of kinetic energy to internal energy remained low. As stated in Section 2.4.4, a maximum ratio of about 5\% is deemed to be acceptable. As shown in Figure 4-7, the internal energy is significantly greater than the kinetic energy. In fact, kinetic energy at the end of the time step was only $1.4 \%$. Because of this, the solution is deemed to be a quasi-static response. 


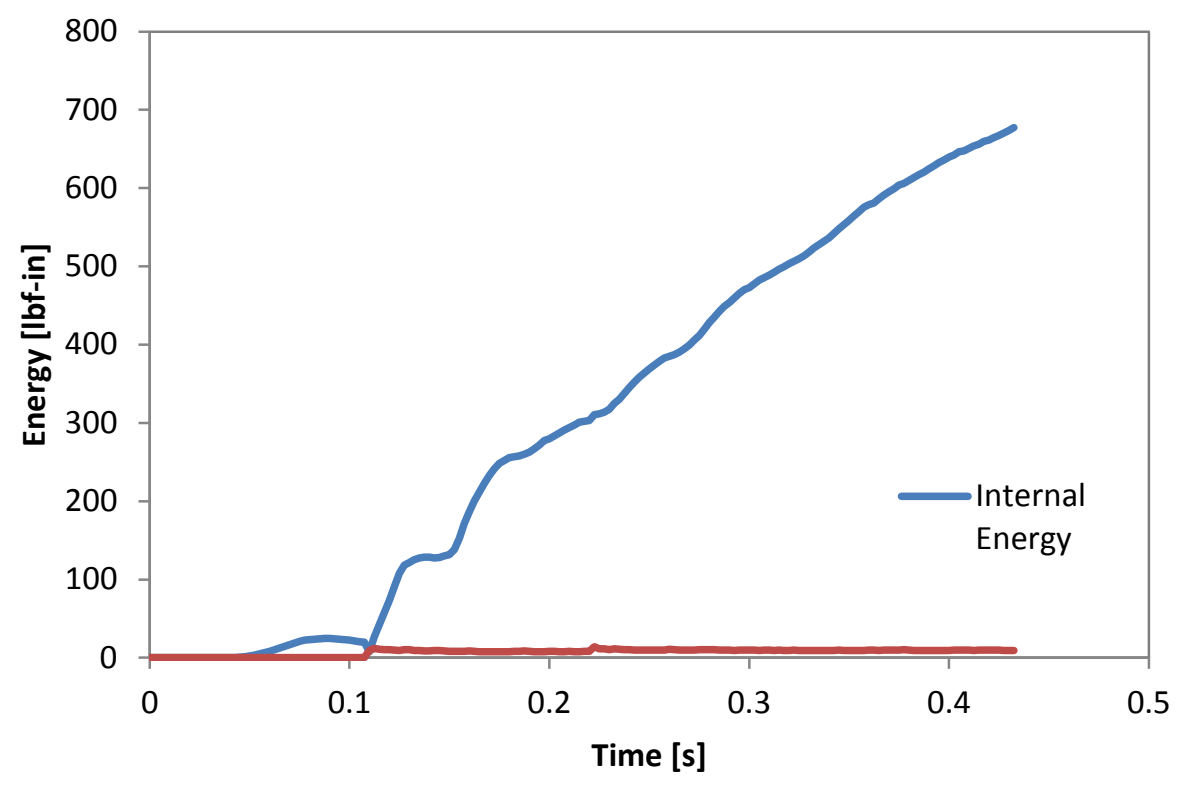

Figure 4-7 - Internal and kinetic energy for the entire tube crush model through the analyzed time period.

Next, a force versus displacement plot was generated and compared to that of the test subjects. Figure 4-8 below shows the response curves.

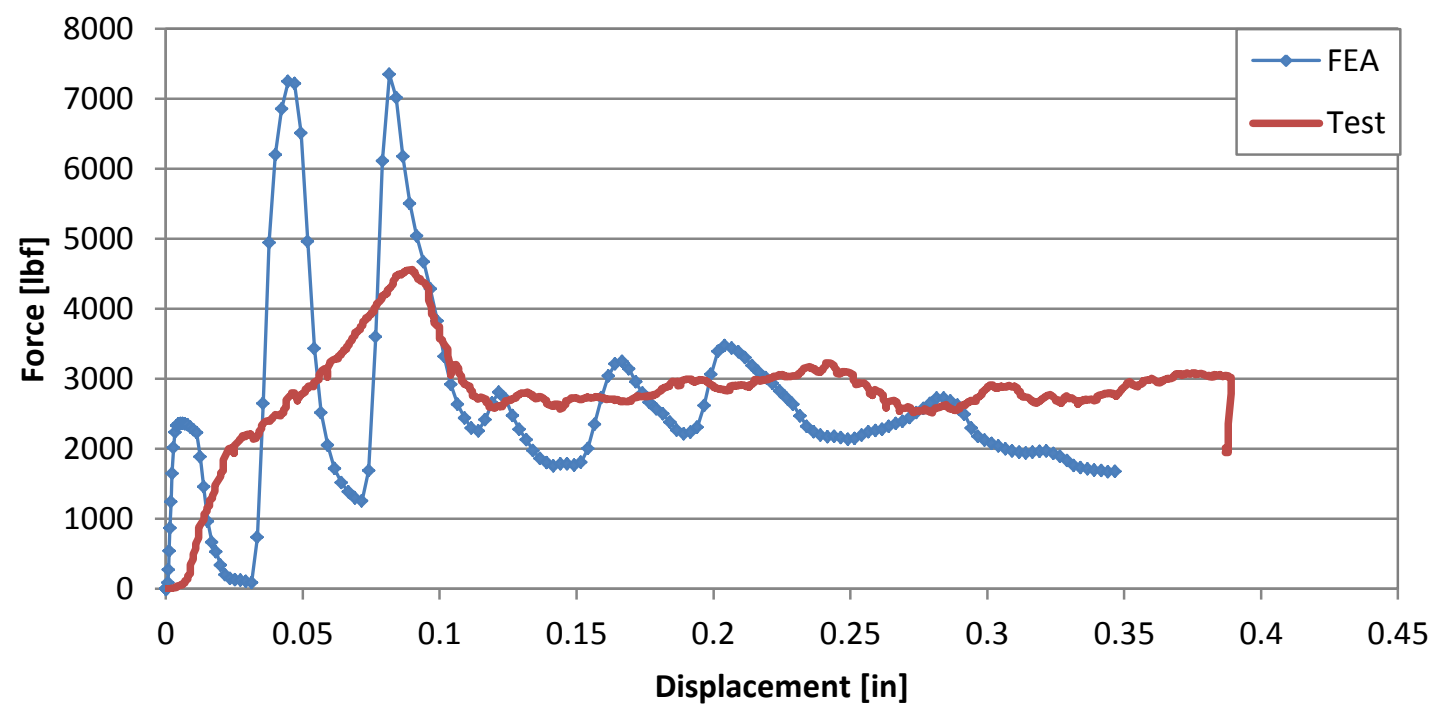

Figure 4-8 - Force versus displacement for test subject and FE analysis.

First, it is apparent that the trigger response of the FE model is much more sporadic than the test subject. Looking at the deformed shape at the initial part of the crush (Figure 4-9), it is 
apparent that the sidewall is starting to buckle. This is why there is such a large build up in load, followed by a sudden drop. The tube is being loaded until non-localized buckling occurs and is not experiencing localized buckling at the plate interface.

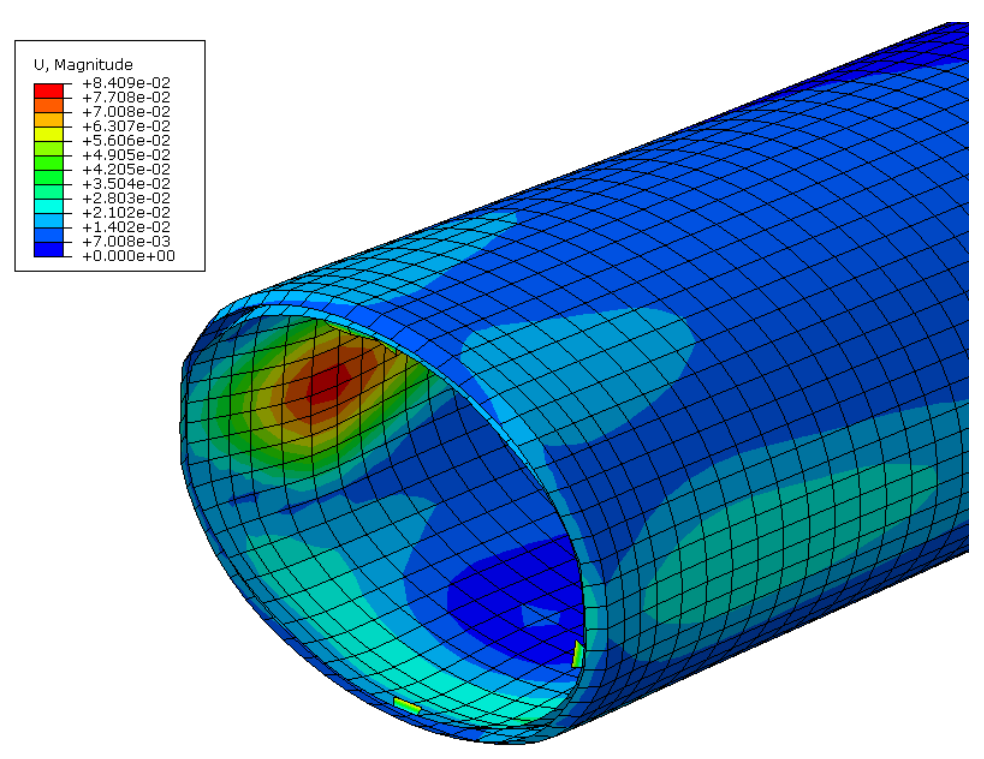

Figure 4-9 - Deformed tube shape with displacement magnitude contour at the initiation of crushing load.

Many different methods were investigated to see if this problem could be mitigated. The max degradation was decreased in order to delete the element sooner so buckling wouldn't occur. However this produced an even more enhanced effect as the trigger was fully damaged very quickly allowing the plate to reinitiate contact with the tube at full speed. This caused the tube to buckle over and over again as evidenced by the sharp peaks in Figure 4-10. 


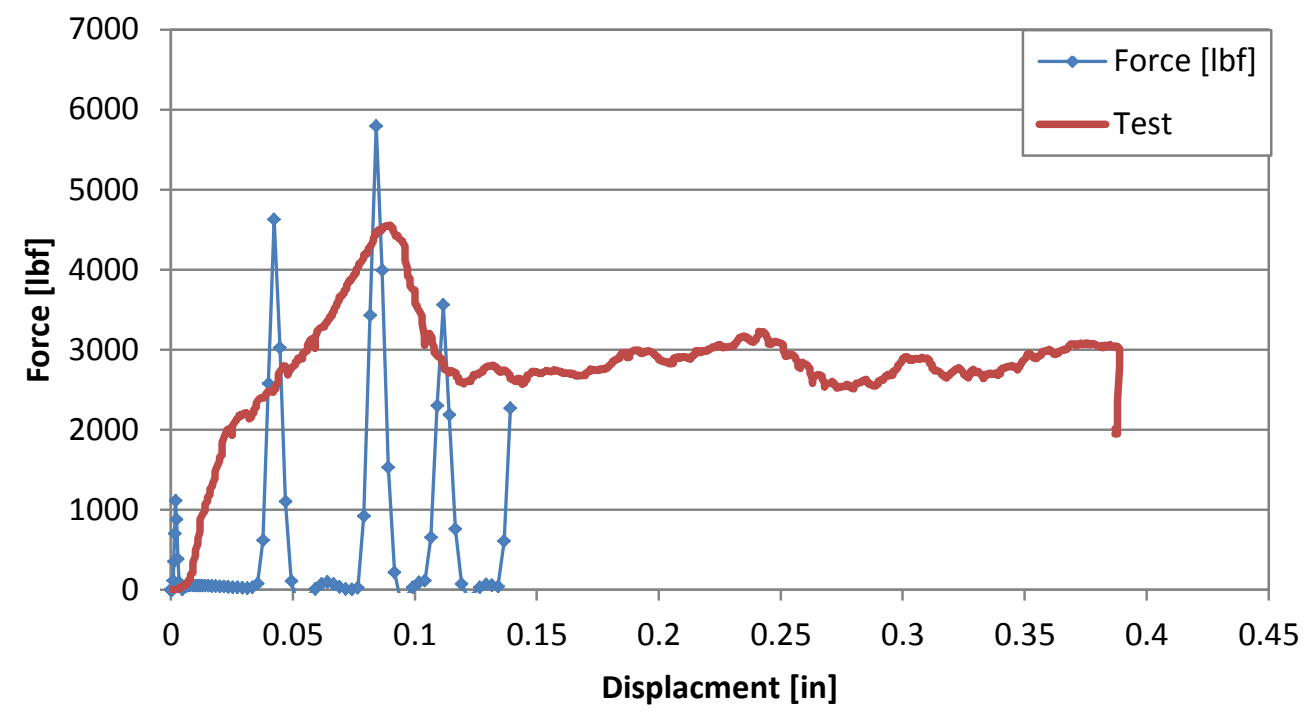

Figure 4-10 - Tube crush with trigger $D_{\max }=0.5,0.2$ in post trigger set to $D_{\max }=0.85$. Run terminated early due to undesirable results.

Next, the bulk viscosity was changed to 0.5 according the Robert's model [14]. The peak trigger load actually increased over the original run, followed by an oscillating force in the stable crush zone. It is apparent that the bulk viscosity did not allow the tube wall to kink or buckle as much as in the previous runs allowing the load to be transferred better. However, the response still doesn't represent what happened during the test.

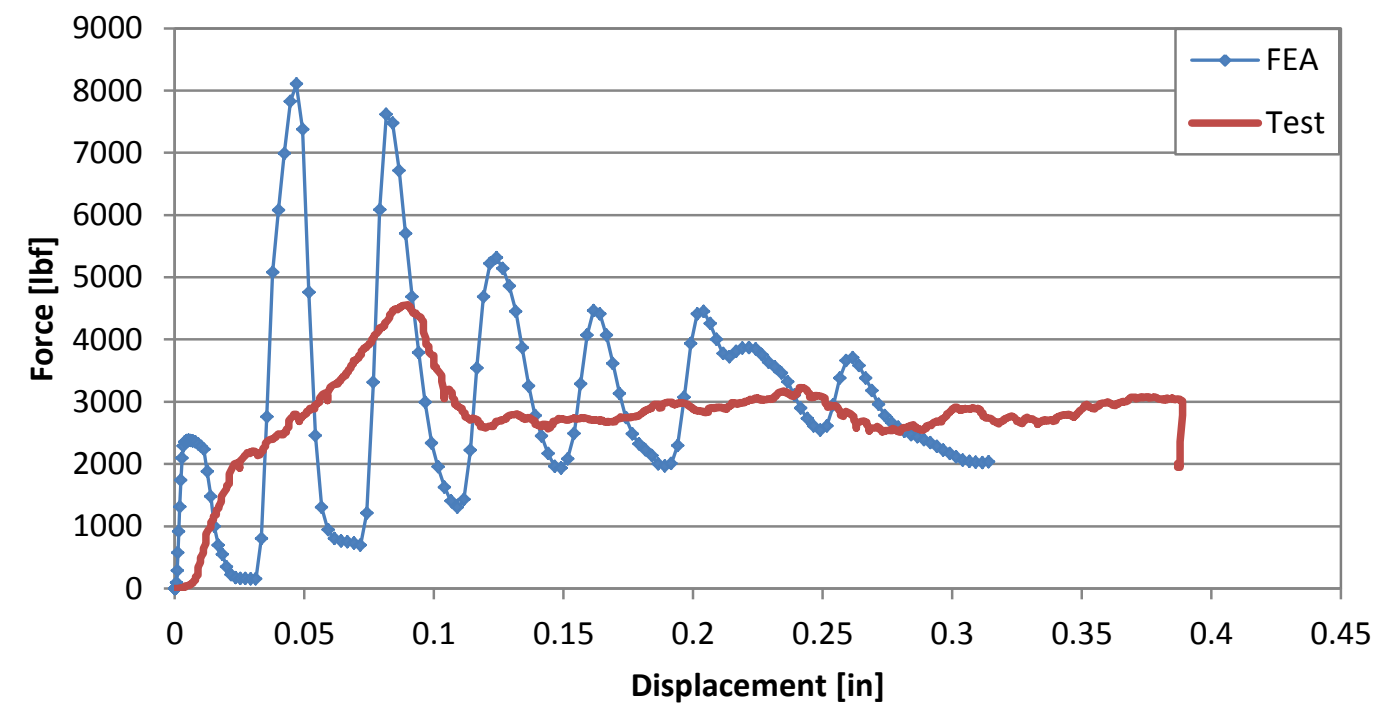

Figure 4-11 - Load vs. displacement for run with bulk viscosity set to 0.5. 
The last attempt to try to improve the response was to reduce the trigger initiation strength by $50 \%$ to simulate any defects caused by manufacturing the trigger. Figure $4-12$ below shows the response. The initial spike decreased by about half over the original run, as expected. However, post initiation ran into the same problems with the tube experiencing buckling away from the plate interface.

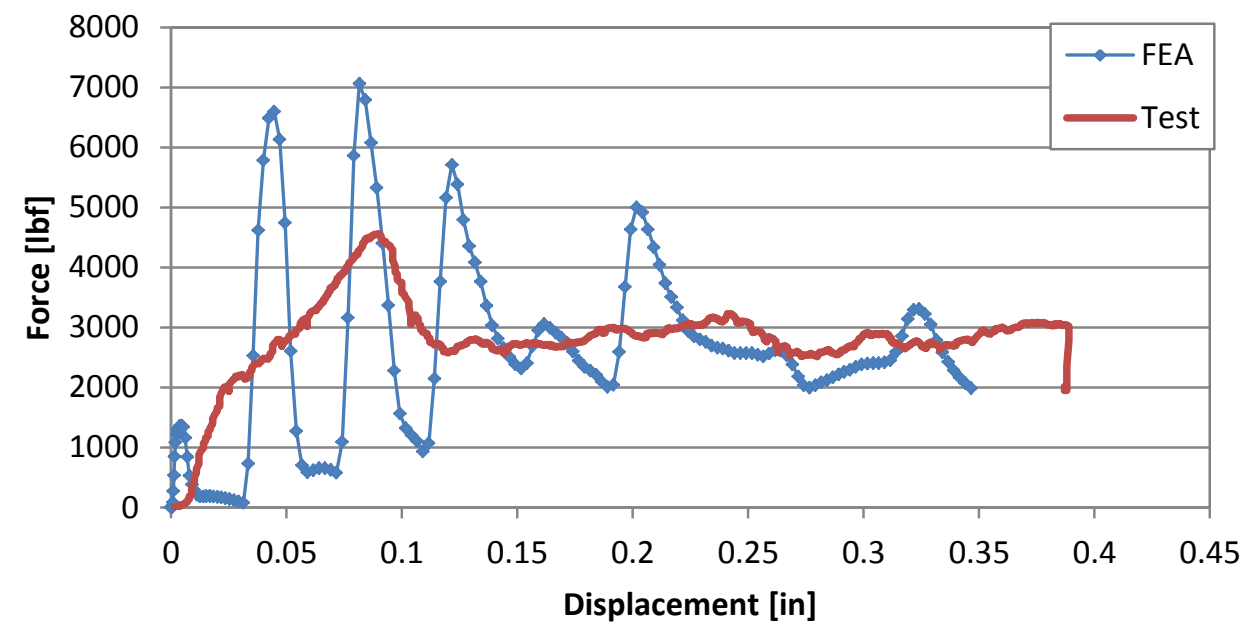

Figure 4-12 - Load vs. displacement for run with 50\% knockdown of damage initiation strength on trigger.

Figure 4-13 below shows the deformed plots for each run. All runs exhibit tube sidewall kinking except for (b) (modified $\mathrm{D}_{\max }$ run), however it should be noted that this plot was taken at a point in time post buckling. It is apparent that this model isn't precisely capturing what is physically happening during the test. 


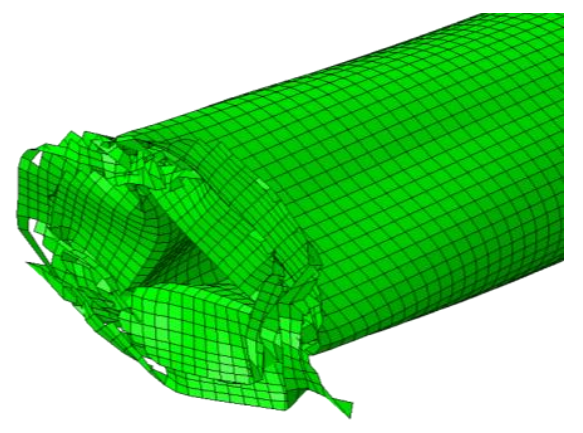

(a)

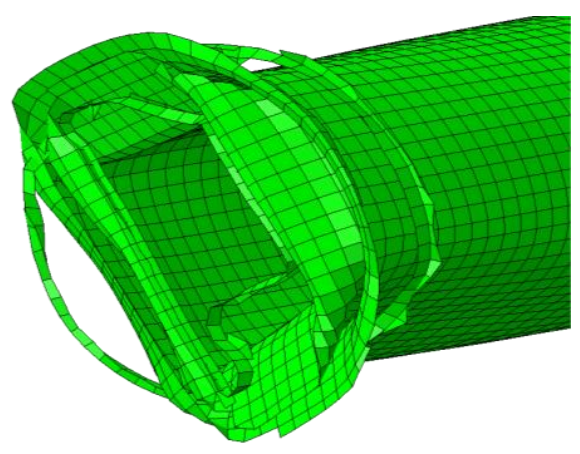

(c)

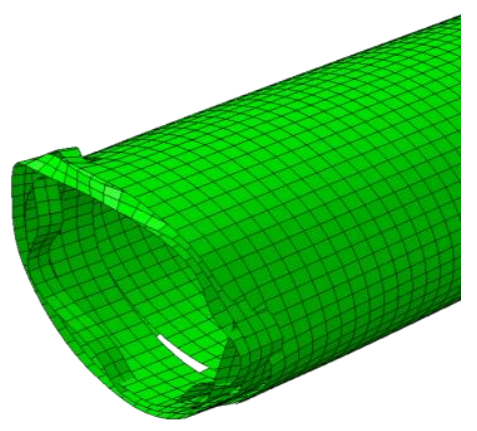

(b)

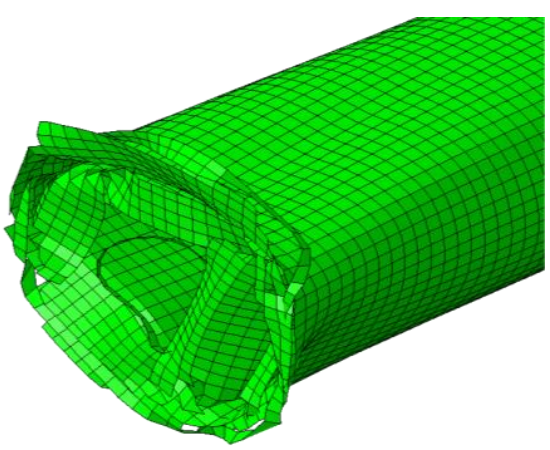

(d)

Figure 4-13 - Deformed plot of (a) original run, (b) modified $D_{\max }$ run, (c) modified bulk viscosity run, and (d) damage initiation strength knockdown run.

Looking at the main difference between this model and Robert's is that the layup is highly aligned. This means that the hoop stiffness is largely dominated by the matrix or transverse properties. The transverse responses of the materials were not very well known because no lab data was available to characterize the damage initiation strengths as well as the fracture energies. In order to achieve a more accurate response, tensile and compression tests would need to be performed on a 90 degree laminate to obtain these values which, unfortunately, are beyond the scope of this paper. This is discussed further in Chapter 6 .

Additionally, upon closer inspection of Figure 4-2, there is evidence of significant delamination occurring. Layers of unidirectional plies can be seen on the damaged frays of the tube. Since this model does not account for delamination, this failure mode would not show up in 
the analysis. A more detailed model might be required, such as one that uses solid continuum shell and cohesive elements to represent each ply and interlaminar bond respectively.

Despite being unable to achieve an accurate tube model, the lessons learned from this study for explicit, quasi-static analyses were taken and applied to the 2013 test nosecone. Seeing as the nosecone has more varied fiber orientations and a "more three dimensional" geometry, the thought was that results would be more achievable than the tube. This is discussed in Chapter 5 . 


\section{CHAPTER 5}

\section{NOSECONE QUASI-STATIC CRUSH MODEL}

The lessons learned from the tube crushing model were applied to the quasi-static crush model of the 2013 test nosecone. This chapter outlines the physical testing that took place, the finite element model development, and the results from the analysis.

\subsection{Quasi-Static Crush Testing of 2013 Nosecone}

A quasi-static crush test was performed at the Cal Poly Civil Engineering Test Laboratory. Figure 5-1 below shows a schematic of the nosecone. A representative nosecone was constructed from unidirectional CFRP. The layup consisted of 0 degree and 90 degree plies using C30/AF254 CFRP. Many layups were trialed during the testing period in an effort to meet the requirements put forward by SAE for the FSAE Collegiate Design Series. Table 5-1 below outlines the final ply layup schedule for the nosecone.
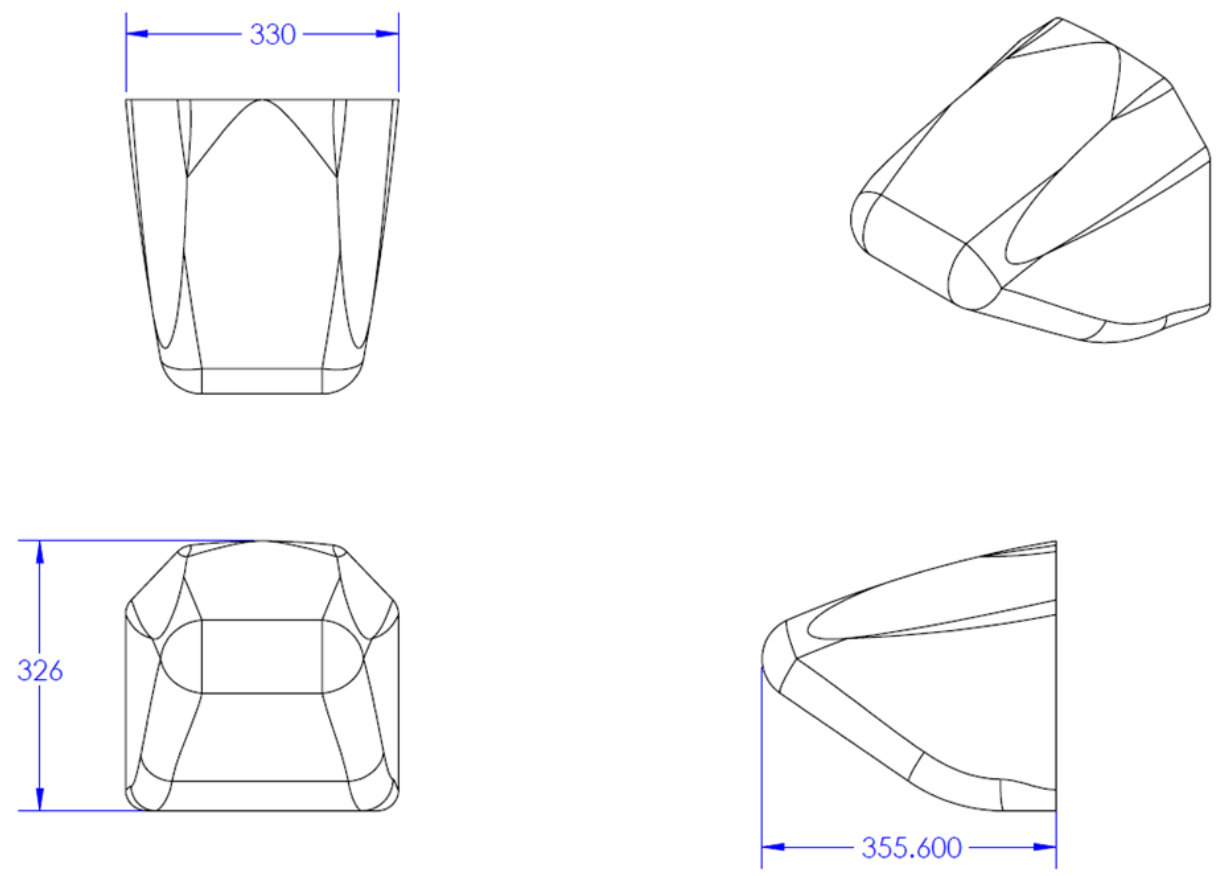

Figure 5-1 - Schematic of the 2013 Nosecone. Dimensions shown are in millimeters. 
Table 5-1 - Layup schedule for the 2013 Cal Poly FSAE Nosecone.

\begin{tabular}{|c|c|c|}
\hline Layer & Orientation & Region \\
\hline 1 & 0 & Sides, Top, Bottom \\
\hline 2 & 90 & Sides, Top, Bottom \\
\hline 3 & 0 & Sides, Top, Bottom \\
\hline 4 & 90 & Sides, Top, Bottom, Cap \\
\hline 5 & 0 & Sides, Top, Bottom, Cap \\
\hline 6 & 90 & Sides, Top, Bottom, Cap \\
\hline 7 & 0 & Sides, Top, Bottom \\
\hline 8 & 90 & Sides, Top, Bottom \\
\hline 9 & 0 & Sides, Top, Bottom \\
\hline 10 & 90 & Sides \\
\hline 11 & 0 & Sides \\
\hline 12 & 90 & Sides \\
\hline 13 & 0 & Sides \\
\hline 14 & 90 & Sides \\
\hline 15 & 0 & Sides \\
\hline 16 & 90 & Sides \\
\hline 17 & 0 & Sides \\
\hline 18 & 90 & Sides \\
\hline
\end{tabular}

The nosecone was then placed in a steel test fixture with a representative aluminum antiintrusion plate per FSAE rules. The fixture was placed in a MTS test machine. A steel plate was attached to the impactor piston and leveled. The impactor plate was displacement controlled at a rate of approximately $25.4 \mathrm{~mm} / \mathrm{min}$. The reaction force of the impactor piston and the displacement were recorded at a rate of approximately $4 \mathrm{~Hz}$. It should also be noted that the test fixture was raised at a displacement of $145.9 \mathrm{~mm}$. This was due to the capacity of the stroke of the MTS test machine. For more information, see Appendix A for the test report. 


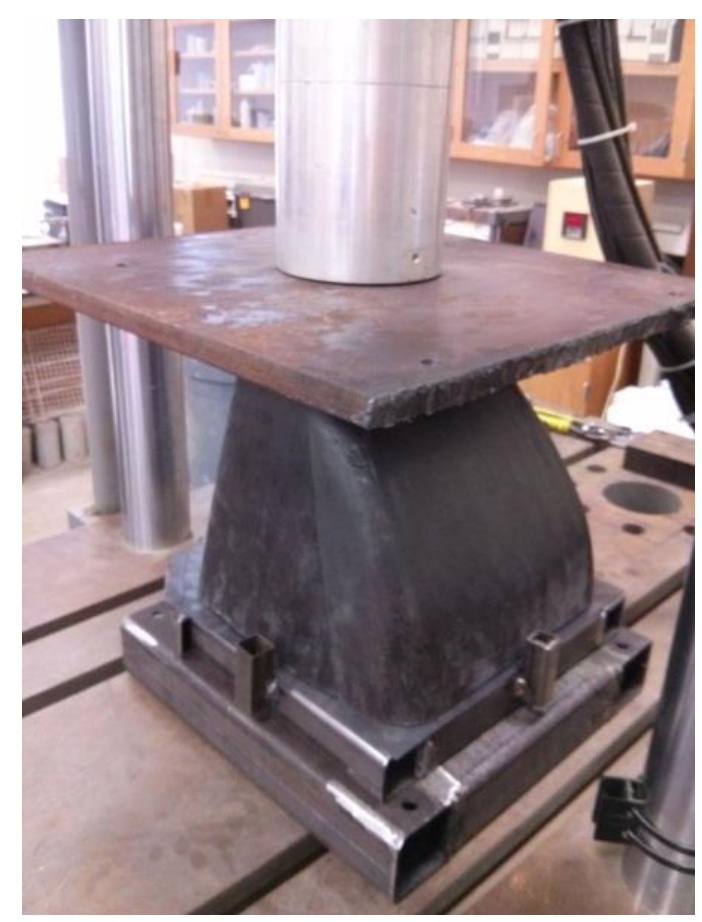

Figure 5-2 - Nosecone loaded into MTS test machine prior to quasi-static crush.

The nosecone met required energy absorption of $7350 \mathrm{~J}$ after approximately $147 \mathrm{~mm}$ of displacement. Figure 5-3 and Figure 5-4 below show the load versus deflection and deceleration and energy absorbed respectively. Note that acceleration calculations assumed a $300 \mathrm{~kg}(661 \mathrm{lb})$ combined car and driver weight.

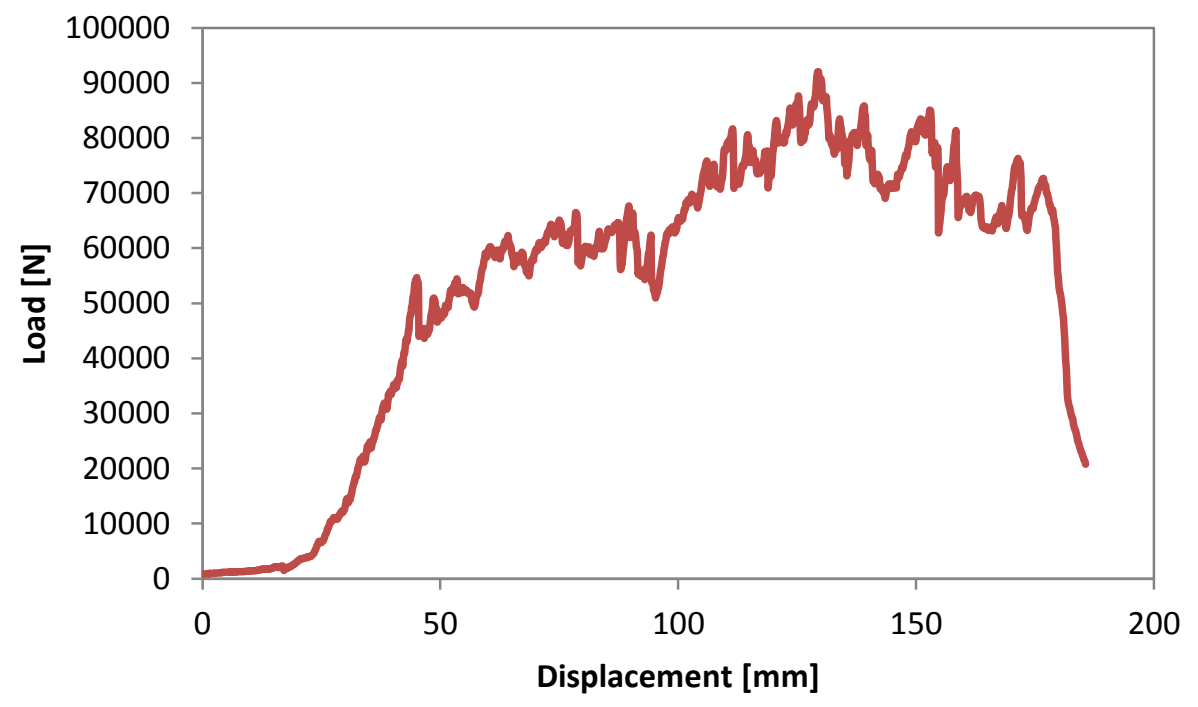

Figure 5-3 - Load versus displacement for quasi-static crush test of the 2013 nosecone. 


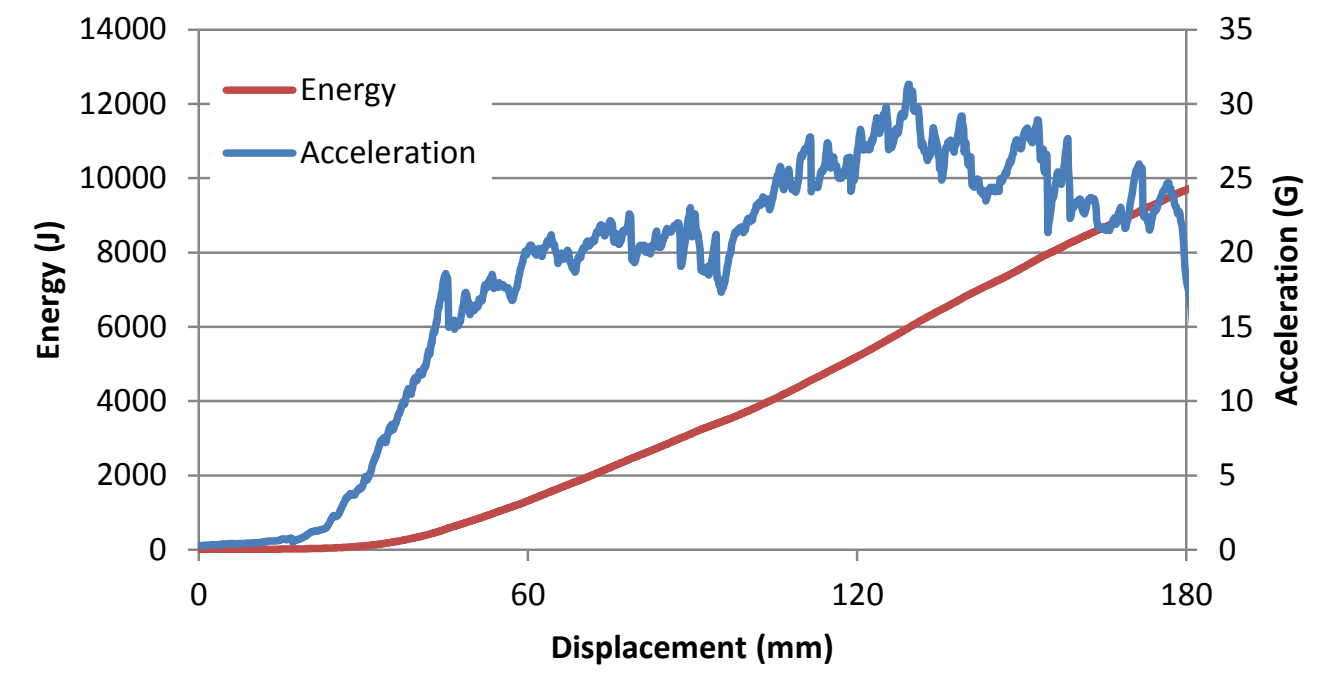

Figure 5-4 - Energy absorption and deceleration versus displacement from the quasi-static nosecone crush test.

There are several metrics that the impact attenuator must meet in order to pass the requirements set forth by SAE. These include energy absorption as described above, a peak deceleration no greater than $40 \mathrm{~g}$, and an average deceleration no greater than $20 \mathrm{~g}$. The 2013 nosecone passed all of these metrics, absorbing $7350 \mathrm{~J}$ of energy at $147 \mathrm{~mm}$ of displacement, having a peak deceleration of $31.3 \mathrm{~g}$ and an average deceleration of $17.0 \mathrm{~g}$. Note that the average deceleration was determined from data taken up to $147 \mathrm{~mm}$ of displacement, as the energy absorbed up to this point was enough to stop a $300 \mathrm{~kg}$ vehicle traveling at a rate of $7 \mathrm{~m} / \mathrm{s}$ as described by the FSAE rules.

Assessment of the damage done during the quasi-static testing revealed that the sides of the nosecone were doing a majority of the work. As shown in Figure 5-5 below, the bottom remains relatively intact while the sides exhibit major fiber damage. Also, the cap remained intact although some matrix cracking was observed that traversed along the transverse fibers. In addition to the sidewall damage, some fiber damage was observed along the base of the nosecone. 


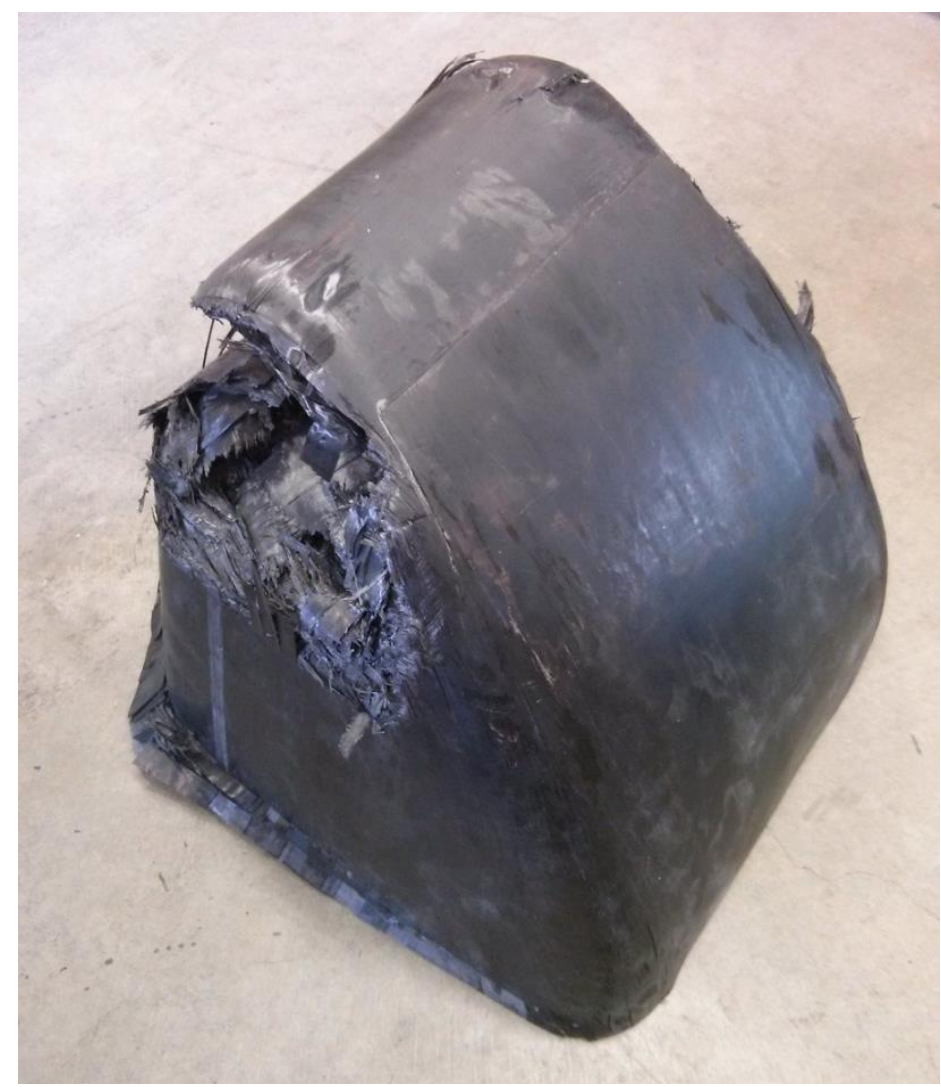

Figure 5-5 - Nosecone post quasi-static crush test.

\subsection{Finite Element Model Development}

A finite element model was created in an effort to recreate the results described in the testing above. Surface geometry was taken from SolidWorks and imported into Abaqus v6.13. A mesh was created using a combination of fully integrated and reduced integrated linear shell elements (S4 and S4R respectively). The fully integrated elements were located in the trigger, while the reduced integrated elements made up the body. The element size was approximately 7.5 $\mathrm{mm}$. A mesh density study was not conducted as this element size provided adequate results with feasible run times. Figure 5-6 below shows an overview of the mesh. The layup was defined as in the previous section. Material properties for T700/E765 were used since full material properties for the C30/AF254 CFRP were not readily available and the materials were comparable (similar fiber strength, 250F cure epoxy resin system). Table 5-2 below shows the material properties used in the finite element model. 


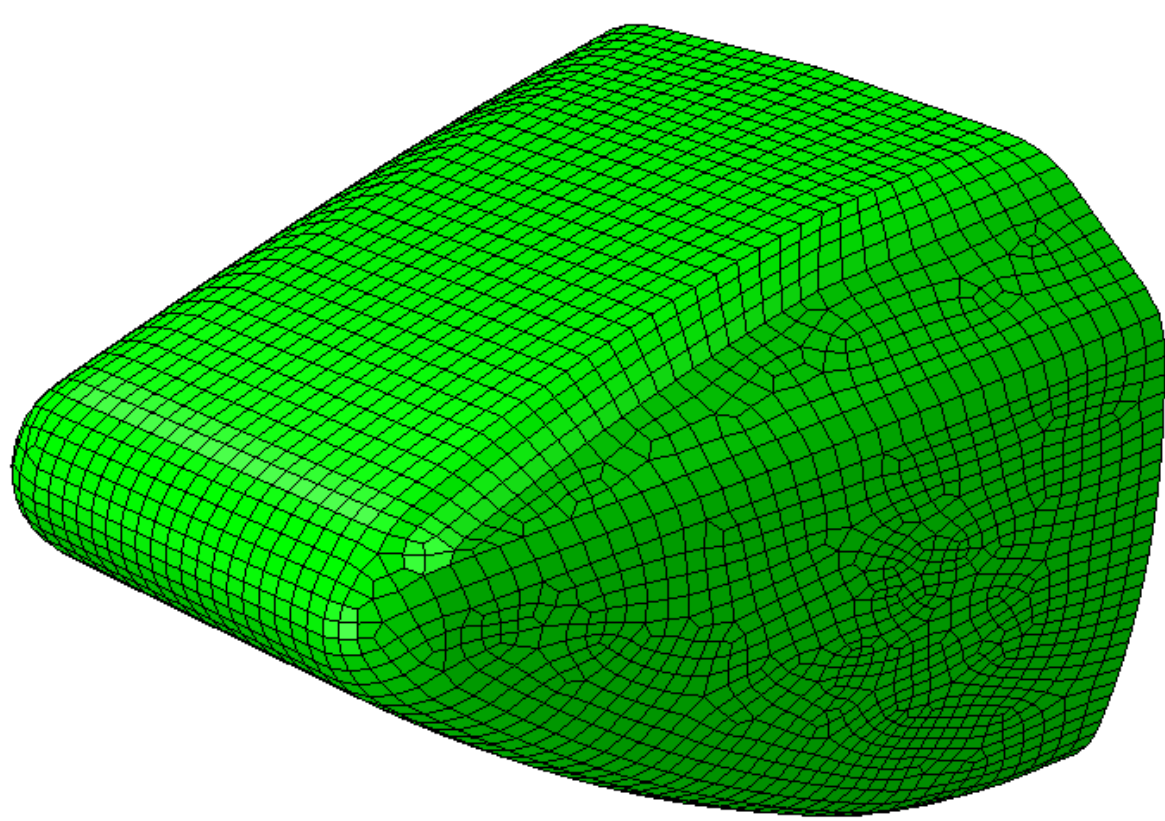

Figure 5-6 - Overview of the Test Nosecone mesh. 
Table 5-2 - Material properties for T700/E765.

\begin{tabular}{|c|c|c|c|}
\hline & Property & Units & Value \\
\hline & Density & tonne $/ \mathrm{mm}^{3}$ & $1.548 \mathrm{E}-04$ \\
\hline \multirow{6}{*}{$\frac{\vec{E}}{\sqrt[3]{11}}$} & E1 & $\mathrm{MPa}$ & 129005 \\
\hline & E2 & $\mathrm{MPa}$ & 9377 \\
\hline & $v 12$ & -- & 0.319 \\
\hline & G12 & $\mathrm{MPa}$ & 4482 \\
\hline & G13 & $\mathrm{MPa}$ & 4482 \\
\hline & G23 & $\mathrm{MPa}$ & 4482 \\
\hline \multirow{7}{*}{ 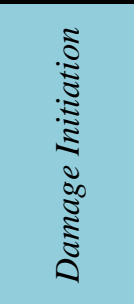 } & $\mathrm{Xt}$ & $\mathrm{MPa}$ & 2553 \\
\hline & $\mathrm{Xc}$ & $\mathrm{MPa}$ & 1239 \\
\hline & Yt & $\mathrm{MPa}$ & 42 \\
\hline & Yc & $\mathrm{MPa}$ & 200 \\
\hline & $\mathrm{Sl}$ & $\mathrm{MPa}$ & 138 \\
\hline & $\mathrm{St}$ & $\mathrm{MPa}$ & 138 \\
\hline & $\alpha$ & -- & 0 \\
\hline \multirow{4}{*}{ 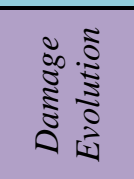 } & Glt & $\mathrm{mJ} / \mathrm{mm}^{2}$ & 20040 \\
\hline & Glc & $\mathrm{mJ} / \mathrm{mm}^{2}$ & 5658 \\
\hline & Gtt & $\mathrm{mJ} / \mathrm{mm}^{2}$ & 143 \\
\hline & Gtc & $\mathrm{mJ} / \mathrm{mm}^{2}$ & 2395 \\
\hline \multirow{4}{*}{ 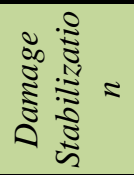 } & $\eta \mathrm{lt}$ & -- & N/A \\
\hline & $\eta \mathrm{lc}$ & -- & N/A \\
\hline & $\eta \mathrm{tt}$ & -- & N/A \\
\hline & $\eta t c$ & -- & N/A \\
\hline
\end{tabular}

In addition to the nosecone, a rigid plate was also modeled using an analytical rigid surface. This defines a plane in which the displacements are described by an analytical function. Because of this, the plate does not need to be meshed. This was done under the assumption that the impactor plate is quite a bit stiffer than the nosecone and can be regarded as rigid.

A general contact domain was defined that included the nosecone and the plate. Abaqus/Explicit can use a domain based definition for the contact algorithm as opposed to a surface-to-surface definition. In other words, Abaqus automatically defines all of the surface-tosurface contact definition to all of the surfaces that are included in the domain. Specific surfaceto-surface contact definitions can be applied in addition to the domain contact definitions. The impactor and nosecone surface had three non-default contact definitions defined. First, the tangential behavior was set using a penalty friction formulation with a friction coefficient of 0.1 . 
Next, a "hard" contact pressure-overclosure was defined so damping could be applied. Finally, a damping coefficient of 0.2 was used to smooth the response of the analysis.

Next the boundary conditions were defined. The base of the nosecone was fixed in the longitudinal degree of freedom. The top and bottom edges were constrained in the $\mathrm{X}$ direction while the side edges were constrained in the $\mathrm{Y}$ direction. All of the rotational degrees of freedom were released. The impactor plate was assigned a velocity of $50 \mathrm{~mm} / \mathrm{s}$ to be ramped up using a smooth step amplitude with a time period of 0.15 seconds. The solution time period was set to 4 seconds. This would allow the impactor to travel approximately $200 \mathrm{~mm}$, which would capture the response that was tested.

\subsection{Results and Discussion}

The analysis was performed on the Cal Poly Mechanical Engineering server running Abaqus v13 in Windows Server 2008 R2 Standard using twin Intel Xeon $2.80 \mathrm{GHz}$ processors and 16 GB of RAM. The model took approximately 4.5 hours to complete.

After successful completion of the analysis, the first check was to observe the deformed shape. Figure 5-7 below shows the crushed nosecone with the impactor plate removed for clarity. It is apparent that the sides, top, and bottom have folded in on itself. It is difficult to compare this shape with reality since an impactor plate was in the way of visually verifying this deformed shape. 


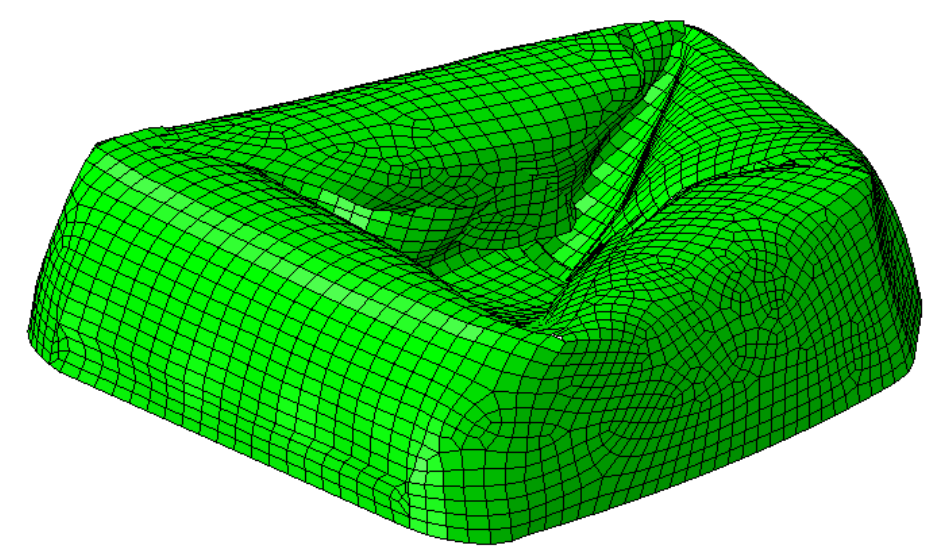

Figure 5-7 - Final deformed shape of nosecone FE model.

When the nosecone was removed from the test fixture, the undamaged sides rebounded to its original shape (see Figure 5-5). Figure 5-8 below shows the undeformed nosecone with fully damaged elements removed. The damage patterns observed were very similar, with full damage occurring along the transition between the sides and top/bottom. Additionally, as shown in Figure 5-9 below, the fiber compressive damage pattern further matches the test results. The most damage is seen on the nosecone sides in the transition regions between layup schedules. Since the deformed shape and damage pattern matched testing, the model passed the first visual check.

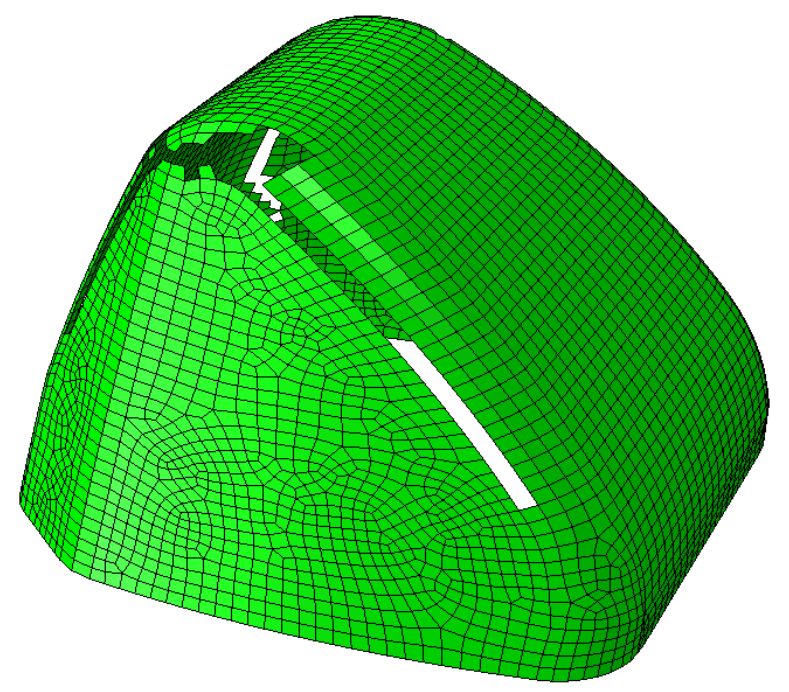

Figure 5-8 - Undeformed nosecone shape with fully damaged elements removed. 


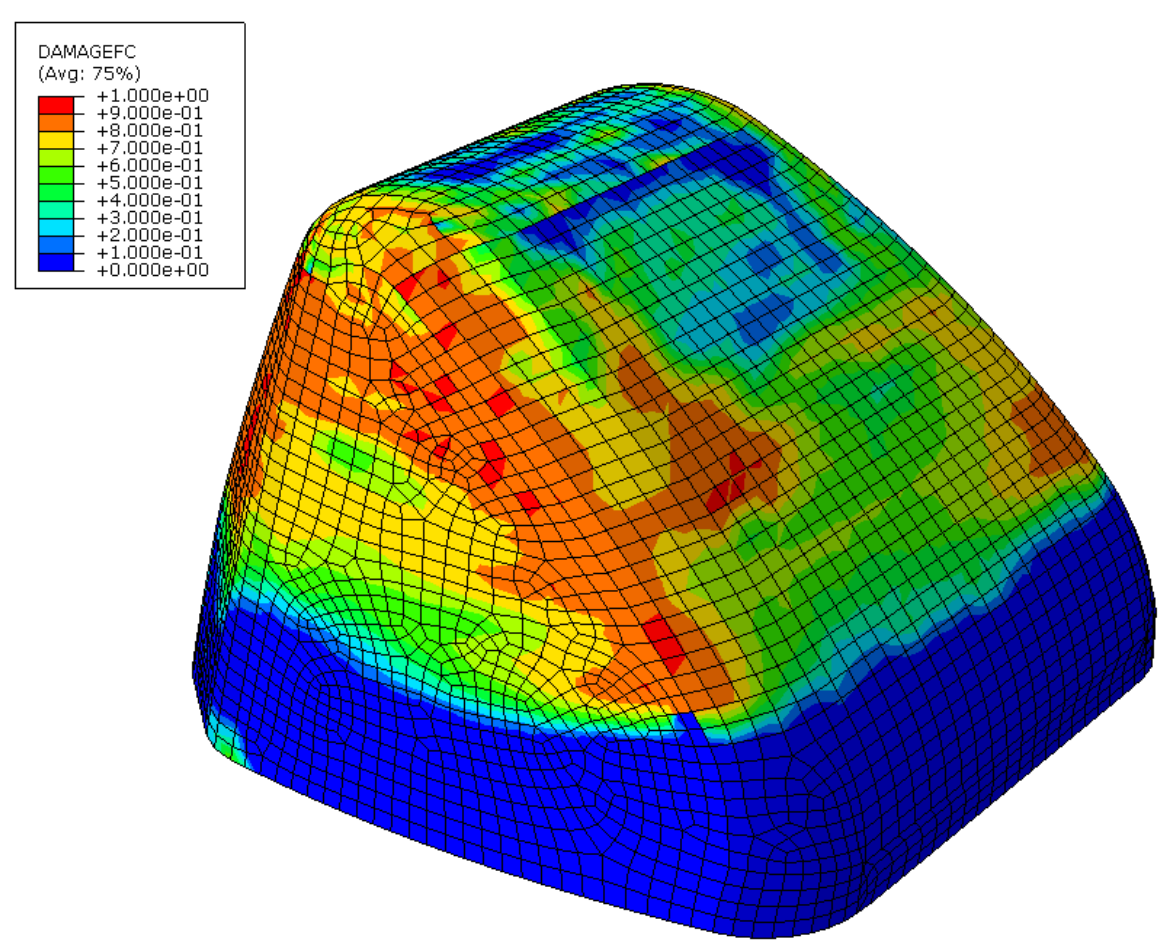

Figure 5-9 - Enveloped fiber compressive damage of the crushed nosecone.

Next, the internal and kinetic energies were checked to ensure a quasi-static response. As stated in the previous section, the ratio of kinetic to internal energy should be no more than $5 \%$ at the end of the analysis. Figure 5-10 below shows the internal and kinetic energies with respect to time. As shown, the internal energy clearly dominates the response. At the end of the analysis, the ratio of kinetic to internal energy was $4.1 \%$. This is under the recommended $5 \%$, therefore the analysis can be considered a quasi-static response. 


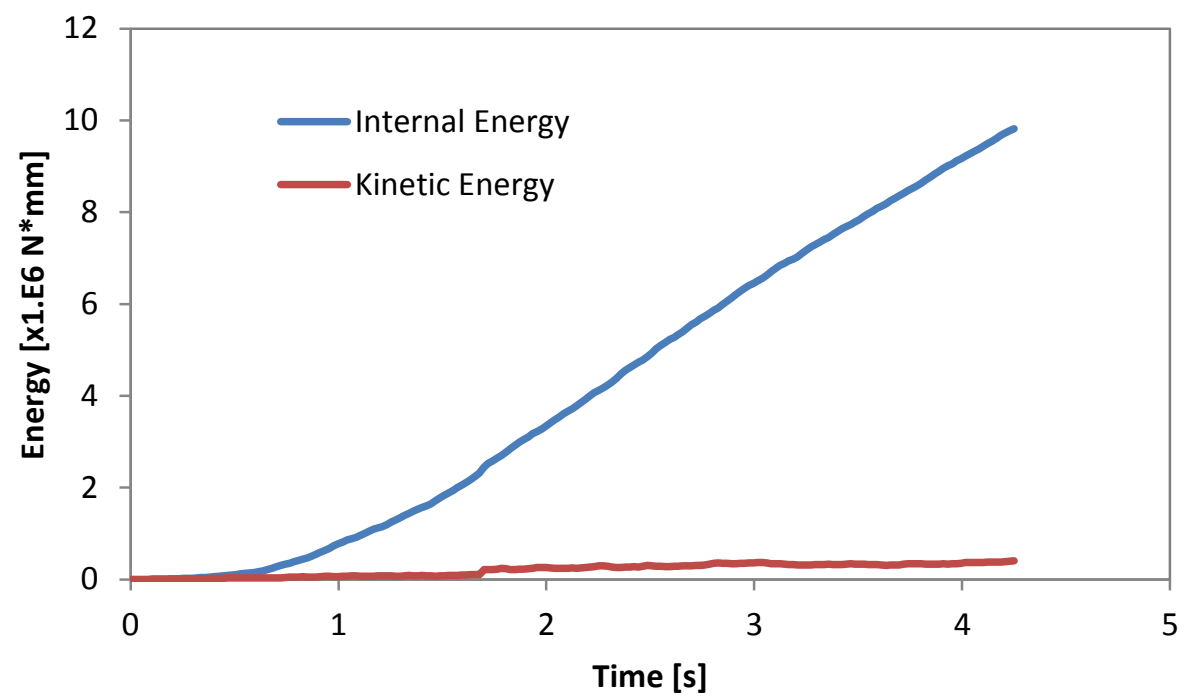

Figure 5-10 - Internal and kinetic energies of the nosecone crush model with respect to time.

Next, in order to prove model validity, the load versus deflection curves of the model were compared with the test results in Section 5.1. Figure 5-11 below shows the two loaddeflection curves. The FE results showed remarkable similarity to the test results save for the severity of oscillations in the response. This was somewhat expected based on the results Roberts obtained [14].

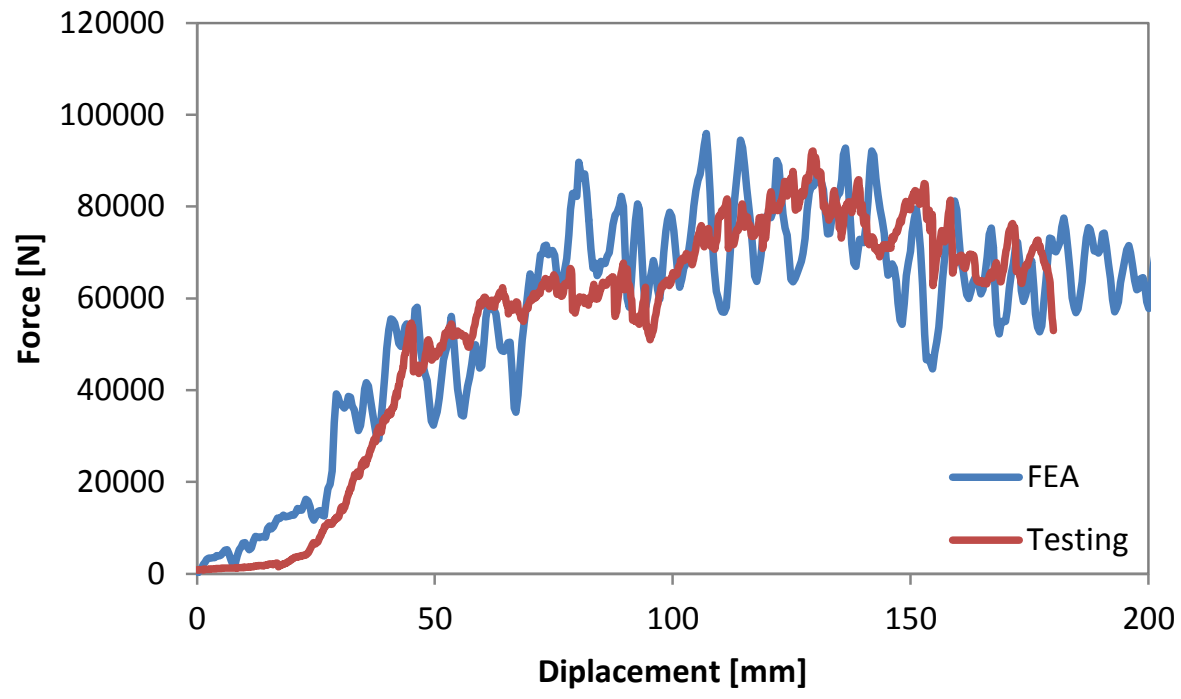

Figure 5-11 - Load versus deflection curves from FEA and testing. 
Next, the impact performance was assessed. As stated in Chapter 1, the nosecone is required to be able to stop a $300 \mathrm{~kg}$ car traveling at $7 \mathrm{~m} / \mathrm{s}$. In other words, the nosecone must be able to absorb a minimum of $7350 \mathrm{~J}$ of energy while maintaining acceptable average and peak accelerations ( $20 \mathrm{~g}$ and $40 \mathrm{~g}$ respectively). The deceleration can be derived from the force vs deflection curve using (5-1) below where $m=300 \mathrm{~kg}$.

$$
a=\frac{F}{m}
$$

The energy absorbed can be also be derived from the force versus deflection curves using (5-2), where $n$ is the total number of increments and $F_{i}$ and $d_{i}$ are the force and displacement at increment $i$ respectively.

$$
E=\sum_{i=1}^{n}\left(F_{i} *\left(d_{i}-d_{i-1}\right)\right)
$$

Figure 5-12 below shows the deceleration and energy absorption curves from the FE analysis of the nosecone. 


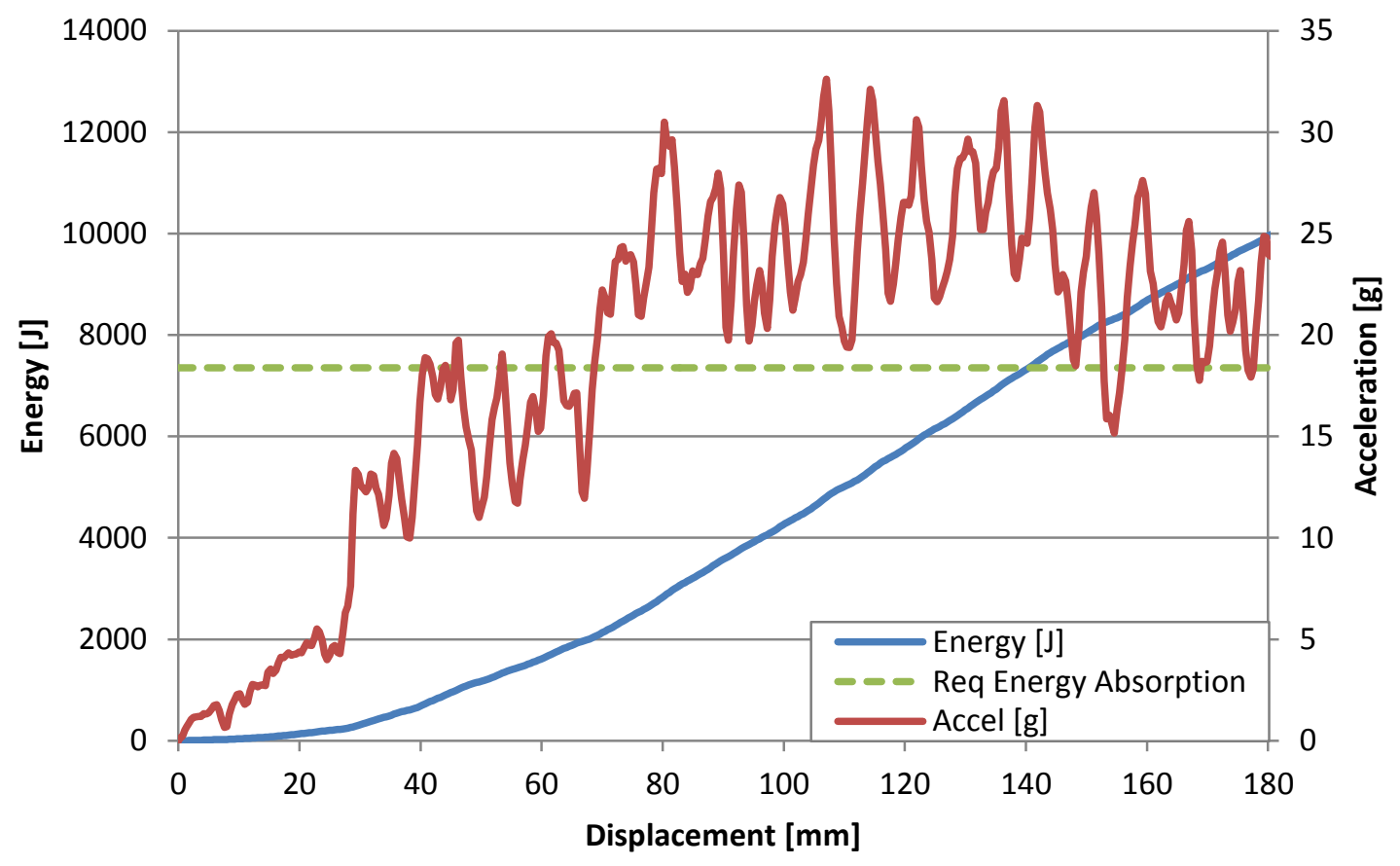

Figure 5-12 - Deceleration and energy absorption of nosecone FE model.

Table 5-3 below shows the impact metrics used to evaluate the nose cone. The FE analysis results were in good agreement with the test results. The displacement at $7350 \mathrm{~J}$ was slightly less than tested. This can be attributed to the higher loads seen at the initial part of the analysis. Since the energy absorption distance was slightly lower, the average deceleration was slightly higher than tested. Also, the peak acceleration was slightly higher than tested.

Table 5-3 - Impact metrics for Finite Element model and test nosecone.

\begin{tabular}{|c|c|c|c|c|}
\cline { 2 - 5 } \multicolumn{1}{c|}{} & Units & FEA & Test & Delta \\
\hline Disp @ 7350 J & $\mathrm{mm}$ & 141 & 147 & $-4.1 \%$ \\
Peak Accel & $\mathrm{g}$ & 32.6 & 31.3 & $4.2 \%$ \\
Average Accel & $\mathrm{g}$ & 17.2 & 17.0 & $1.3 \%$ \\
\hline
\end{tabular}

While the model showed good agreement with the test results, there are some known discrepancies between the FEM and test article that should be noted. First, since the elements had to be defined at the mid-plane, the ply stacking between layup regions is slightly incorrect. Originally, the nodal plane was defined at the tool surface, such that there would be no "steps" on 
the outer surface of the nosecone. Defining the nodes at the mid-plane of the laminate caused this step to occur in the model. This could be a contributing factor as to why the results were skewed towards a stiffer response.

Second, the nosecone base fixture was not modeled in an effort to simplify the boundary conditions. The fixture allowed the nosecone base to deform more than the defined boundary conditions of this analysis (see Figure 5-2). This may have artificially stiffened the structure and contributed to the stiffer response observed in the analysis.

Third, there is some uncertainty as to the values for fracture energy that were used in this analysis. While they were obtained by the method outlined in Appendix A in [14], other studies have shown that lab results can vary from this theory. This will further be explained in the following section.

Despite these discrepancies, the model still showed good agreement with the test results. Impact metrics of the analysis model were within $5 \%$ of tested values. Additionally, with a reasonable run time of 4.5 hours, this model and analysis method can be used in the future to quickly iterate designs to achieve a lightweight impact attenuator. 


\section{CHAPTER 6}

\section{FUTURE WORK}

While the goal of developing a working model of the nosecone crush test was achieved, there are numerous opportunities for further development in utilizing the Hashin Damage model in Abaqus for impact attenuator design. Specifically, this chapter will discuss obtaining fracture energy values for materials and optimization studies.

\subsection{Fracture Energy}

Without question, one of the largest sources of uncertainty in the models developed in this study is the fracture energy values associated with the damage evolution portion of the material model. As stated previously, these values are determined using the assumption that the material fails like a "perfectly" brittle material (i.e. no stiffness exists in the material once the strength is realized). While this is a decent assumption for CFRP's, this assumption may not be valid for other types of fiber reinforced plastics. Further, while the transverse failure mode is secondary to fiber failure modes, it is unknown whether or not this failure mode can be treated as perfectly brittle.

According to Pinho et al., there is no standard test or experiment to determine the fracture energy of a composite [21]. To obtain these values, the authors used a compact tensile (CT) and compact compression (CC) tests to obtain fiber tensile and compressive fracture energies. The tests utilized photogrammetry to record the strain fields of specimens during the tests in order to check for damage not readily visible in the specimens and to locate the crack tip. Figure 6-1 below shows the schematics of the specimens used in the tests. 


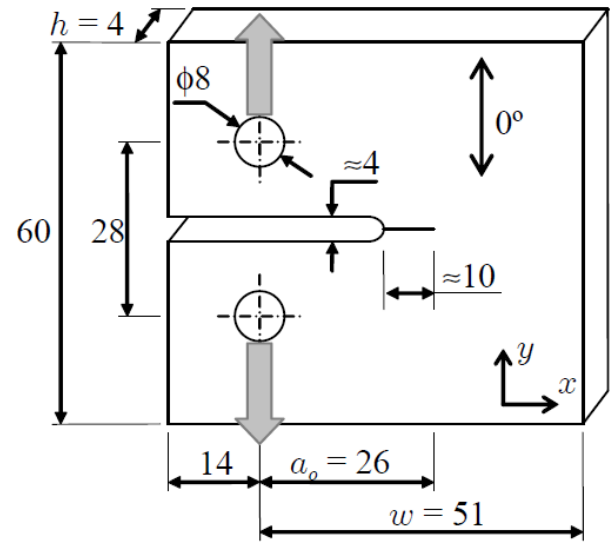

(a) Compact Tension

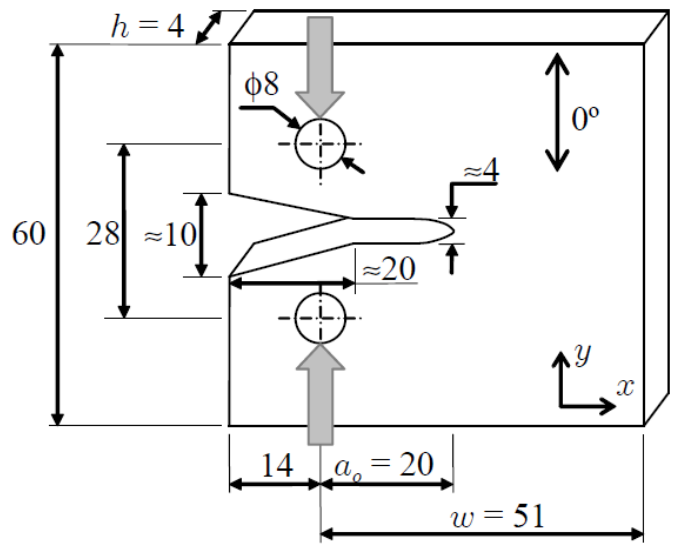

(b) Compact Compression

Figure 6-1 - Compact tension (a) and compact compressions (b) test specimens used in Pinho et al's experiments [21].

The authors were able to determine fracture initiation energies for both the tensile and compressive failure modes and fracture propagation energy for the tensile specimen. No meaningful results for compressive crack propagation were obtained due to the fact that the test produced other significant failure modes other than fiber kinking (significant delaminations were found beyond the crack propagation plane).

These values were later used in developing a failure model for use in explicit Finite Element analysis [9] [10]. This failure mode is similar to Hashin's damage model found in Abaqus, however this model accounts for inter-laminar failure as well. They found that the fracture initiation energies correlated well when implemented into the damage model. It should be noted that the authors utilized solid elements for this study.

For future work, it is suggested that an experimental method for obtaining fracture energies for use with the Hashin Damage model be developed and verified. This way, fracture energies would be based on empirical results rather than an ideal assumption. Also, the use of solid (or continuum shells in Abaqus) elements in place of shell elements could be investigated. 
Solid elements may give more accurate results and would provide more insight into the damaged state of the laminate as each layer would have at least one layer of elements.

\subsection{Optimization}

While the 2013 nosecone met the metrics of the 2013 FSAE rules, no optimization studies were performed. The shape was chosen based on the aesthetics of the car and the layup was developed by "trial and error." The layup development especially led to a costly and lengthy development process that required numerous tests to prove adequacy. With a working model now, an optimization study can take place using FEA. This opens the door for shape, material selection, and ply stacking optimization with the goal of minimizing weight.

For a shape study, two approaches could be taken: a qualitative approach and an algorithm approach. A qualitative approach would consist of crushing different crash structures of different shapes with the same material and layup schedules. This way, general shapes can be characterized on their specific energy absorption (for example, $\mathrm{J} / \mathrm{kg}$ ) and crush load efficiency. A second, much more complicated way would be to utilize an algorithm similar to Lanzi et al [13].

In their study, they performed a multi-objective optimization on conical crash structures by creating response surfaces for vertical, 20 degree, and 30 degree impacts. The response surfaces were developed using 30 data points for each impact case. 20 of the 30 data points were derived using a finite element model. The remaining 10 points were used as verification of the interpolation capabilities of the functions used to define the response surfaces. By doing so, the authors were able to perform an optimization maximizing energy absorption and minimizing weight by using the cone's dimensions as the design variables. A similar study could be performed on the FSAE nosecone to determine optimal dimensions such as the base, sidewall slope, and tip area. 
In addition to shape optimization, material selection and layup schedule can also be performed. One way this could be done is via a Design of Experiment or Taguchi Method. This method utilizes an orthogonal array to explore a design space. For example, if there were three parameters that wanted to be explored, and the minimum and maximum values for each parameter are defined, then the user would utilize an "L4" array to define the experiments, as shown below in Table 6-1.

Table 6-1 - Example Taguchi L4 Orthogonal Array.

\begin{tabular}{|c|c|c|c|}
\hline Experiment & P1 & P2 & P3 \\
\hline $\mathbf{1}$ & Max & Max & Max \\
\hline $\mathbf{2}$ & Max & Min & Min \\
\hline $\mathbf{3}$ & Min & Max & Min \\
\hline $\mathbf{4}$ & Min & Min & Max \\
\hline
\end{tabular}

Depending on the number of parameters and test values for each parameter, the appropriate orthogonal array can be selected. This greatly reduces the number of experiments needed to characterize a problem and also provides design sensitivity.

For the nosecone, parameters such as Material Modulus, Compressive Strength, and Percent Axial Plies could be explored using a Taguchi orthogonal array. Design decisions such as candidate layups and material selections could be made from the sensitivity study while only having to perform a handful of analyses. This would result in a lighter weight nosecone that still met the impact metrics of the FSAE rules. 


\section{CHAPTER 7}

\section{CONCLUSION}

In conclusion, the Hashin Damage model for fiber reinforced materials was investigated using a unidirectional tensile coupon model. It was found that the explicit solution was similar to the implicit solution with less dependency on mesh sizing. Additionally, utilizing mass scaling and "Smooth Step Amplitude" load application decreased runtime and reduced the effects of vibration within the model. Too much fiber fracture energy can lead to unrealistic responses, however too little fracture energy does not seem to be detrimental due to the brittle nature of carbon fiber. Finally, the maximum degradation variable affects the post damage initiation response by limiting the "knockdown" of the stiffness of the material and by deleting elements before they are fully damaged.

An attempt to verify Robert's tube model in the lab led to further developments to his model. Smooth step amplitude load application as well as semi-automatic mass scaling helped reduce run-times. Removing element offsets fixed stability issues and removed the problem with excessive element rotations and distortions. Despite these advances in stability, no realistic results could be obtained from the model. This is thought to be because of the highly aligned laminate accentuating the inaccurate transverse material response. Additionally, the test specimens showed signs of delamination. A more detailed model may be required to capture this failure mode.

The lessons learned from the previous studies were applied to the 2013 FSAE test nosecone. The model was able to predict the crushing response with good accuracy. The impact metrics were within $5 \%$ of tested values. Additionally, the crushed shape and damage pattern matched that the test specimen.

Further research is required into the understanding of the Hashin damage model in Abaqus. It is still unclear the best method for obtaining reliable fracture energy values, especially 
for the transverse direction in highly aligned laminates. This is evidenced by Robert's tube model and the nosecone model being able to achieve reasonable results while the tube crush model in this study was unable to accurately predict the response. Despite this, for three dimensional structures utilizing varied fiber directions, the single shell layer Hashin damage model works well. The nosecone model described in this study can be used as a tool to explore attenuator shapes, material selection, and layup schedule. It should be noted, however, that final designs developed using this model should always be validated via testing before being implemented. 


\section{REFERENCES}

[1] SAE International, 2013 Formula SAE Rules, 2013, Official rules for the Formula SAE competition.

[2] G. Savage, "Formula 1 Composite Engineering," Energineering Failure Analysis, pp. 92$115,2010$.

[3] G. Savage, "Development of penetration resistance in the survival cell of a Formula 1 racing car," Engineering Failure Analysis, pp. 116-126, 2010.

[4] G. Savage, I. Bomphray, and M. Oxley, "Exploiting the fracture properties of carbon fibre composites to design lightweight energy absorbing structures," Anales de Mecanica de la Fractura, vol. 20, pp. 512-522, 2003.

[5] J. Obradovic, S. Boria, and G. Belingardi, "Lightweight design and crash analysis of composite frontal impact energy absorbing structures," Composite Structures, no. 94, pp. 423-430, 2012.

[6] H. Zarei, M. Kroger, and H. Albertson, "An experimental and numerical crashworthiness investigation of thermoplastic coposite crash boxes," Composite Structures, no. 85, pp. $245-257,2008$.

[7] Z. Hashin and A. Rotem, "A fatigue failure criterion for fiber reinforced materials," Technion - Israel Institute of Technology, Haifa, 1973.

[8] Dassault Systemes, Abaqus 6.13 Analysis User's Guide, 2013.

[9] S.T. Pinho, L. Iannucci, and P. Robinson, "Physically-based failure models and criteria for laminated fibre-reinforced composites with emphasis on fibre kinking: Part I: Development," Composites: Part A, pp. 63-73, 2006.

[10] S.T. Pinho, L. Iannucci, and P. Robinson, "Physically based failure models and criteria for linated fibre-reinforced composites with emphasis on fibre kinking. Part II: FE implementation," Composite: Part A, pp. 766-777, 2006.

[11] Shen-Yen Chen, "An approach for impact structure optimization using the robust genetic algorithm," Finite Elements in Analysis and Design, no. 37, pp. 431-446, 2001.

[12] J. Forsberg and L. Nilsson, "Evaluation of response surface methodologies used in crashworthiness optimization," International Journal of Impact Engineering, no. 32, pp. 759-777, 2006.

[13] L. Lanzi, L.M.L. Castelletti, and M. Anghileri, "Multi-objective optimization of composite absorber shape under crashworthiness requirements," Composite Structures, no. 65, pp. 433-441, 2004. 
[14] M. Roberts, "Development of a finite element model for predicting the impact energy absorbing performance of a composite structure," California Polytechnic State University, San Luis Obispo, Masters Thesis 2014.

[15] B.D. Agarwal, L.J. Broutman, and K. Chandrashekhara, Analysis and performance of fiber composites. Hoboken: John Wiley, 2006.

[16] N.M. Newmark, "A method of computation for structural dynamics," Journal of Engineering Mechanics, pp. 67-94, 1959.

[17] Abaqus, Inc., ABAQUS/Explicit: Advanced Topics - Lecture 5: Quasi-Static Analyses, 2005, www.imechanica.org.

[18] B. Willis, "Applying Finite Element Analysis with a Focus on Tensile Damage Modeling of Carbon Fiber Reinforced Polymer Laminates," The Ohio State University, Masters Thesis 2013.

[19] TenCate. (2010) Royal Ten Cate Corporation Web Site. [Online]. http://www.tencate.com

[20] J. Huang and X. Wang, "Numerical and experimental investigations on the axial crushing response of composite tubes," Composite Structures, no. 91, pp. 222-228, 2009.

[21] S.T. Pinho, P. Robinson, and L. Iannucci, "Fracture toughness of the tensile and compressive fibre failure modes in laminated composites," Imperial College London, London, 2005.

[22] A. Matzenmiller, J. Lubliner, and R.L. Taylor, "A constitutive model for anisotropic damage in fiber-composites," Mechanics of Materials, pp. 125-152, 1995.

[23] Chiara Bisagni, Giuseppe Di Pietra, Lara Frashini, and Terletti, "Progressive crushing of a fiber-reinforced composite structural components of a Formula One racing car," Composite Structures, no. 68, pp. 491-503, 2005.

[24] Ireneusz Lapczyk and Juan Hurtado. (2006) Progressive Damage Modeling In FiberReinforced Materials. PowerPoint Presentation.

[25] S. Boria and G. Forasassi, "Progressive crushing of a fiber reinforced composite crash-box for a racing car," DYMAT, pp. 725-731, 2009.

[26] S. Taher and S. Ataollahi, "Using a new composite energy-absorber bumper to improve automotive crashworthiness," Curtin University of Technology, Sarawak, Malaysia, 2009.

[27] Thomas J. Wright, "Sensitivity of Hashin damage parameters for notched composite panels in tension and out-of-plane loading," Oregon State University, Corvallis, Masters Thesis 2012. 
[28] Rahul, D. Chakraborty, and A. Dutta, "Optimization of FRP composites against impact induced failure using island model parallel genetic algorithm," Composites Science and Technology, no. 65, pp. 2003-2013, 2005.

[29] G. Jacob, J. Fellers, S. Simunovic, and J. Starbuck, "Energy absorption in polymer composites for automotive crashworthiness," Journal of Composite Materials, vol. 36, no. 7, pp. 814-850, 2002.

[30] J. Tomblin, J. McKenna, Y. Ng, and K.S. Rajo, "B-Basis Design Allowables for EpoxyBased Prepreg FiberCote Graphite Unitape T700 24k / E765," National Institute for Aviation Research, Wichita, 2004.

[31] I. Lapczyk and J.A. Hurtado, "Progressive damage modeling in fiber-reinforced materials," Composites: Part A, pp. 2333-2341, 2007.

[32] M.J. Laffan, S.T. Pinho, P. Robinson, and A.J. McMillan, "Translaminar fracture toughness testing of composites: A review," Polymer Testing, pp. 481-489, 2012. 
APPENDICES 


\section{APPENDIX B-2 \\ 2013 FSAE $^{\mathrm{TM}}$ IMIPACT ATTENUATOR DATA REPORT - Page 1 of 5}

This form must be completed and submitted by all teams no later than the date specified in the Action Deadlines on specific event website. The FSAE Technical Conmittee will review all submissions which deviate from the FSAEN rules and reply with a decision about the requested deviation. All requests will have a confirmation of receipt sent to the team. Impact Attenuator Data (IAD) and supporting calculations mmst be submitted electronically in Adobe Acrobat Format ("pdf). The submissions must be named as follows: schoolname_IAD.pdf using the complete school name. Submit the LAD report as instructed on the event website. For Michigan and California events submit through fsaeonline.com

"In the event that the FSAE Technical Committee recuests additional information or calculations, teams have one week from the date of the request to submit the requested information or ask for a deadline extension

University Name: California Polytechnic State Univ - SLQ Car Number(5) \& Event(s): 047 FSAEIincoln

Team Contact: John Waldrop $\quad$ E-mail Address: iohnwwaldrop@almail com

Faculty Advisor: John Fabijanic E-mail Address: ifabijan a calpoly.edu

\begin{tabular}{|l|l|}
\hline Material(s) Used & Unidirectional carbon fiber prepreg \\
\hline Description of form/shape & Pyramidal skin \\
\hline IA to Anti-Intrusion Plate & IA fastened using four (4) axial M8x1.25 Grade 8.8 bolts to Front \\
mounting method & Bulkbead (AI plate is clamped between LA and Front Bulkbead) \\
\hline Anti-Intrusion Plate to Front & IA fastened using four (4) axial M8x1.25 Grade 8.8 bolts to Front \\
Bulkhead mounting method & Bullkbead (AI plate is clamped between LA and Front Bulkbead) \\
\hline Peak deceleration ( $\left.c=40 \mathrm{~g}^{\prime} 5\right)$ & $31.3 \mathrm{~g}$ \\
\hline Average deceleration $\left(c=20 \mathrm{~g}^{\prime} 5\right)$ & $17.0 \mathrm{~g}$ \\
\hline
\end{tabular}

Confirm that the attenuator contains the mininmm volume $200 \mathrm{~mm}$ wide $\mathrm{x} 100 \mathrm{~mm}$ high $\mathrm{x} 200 \mathrm{~mm}$ long Ves

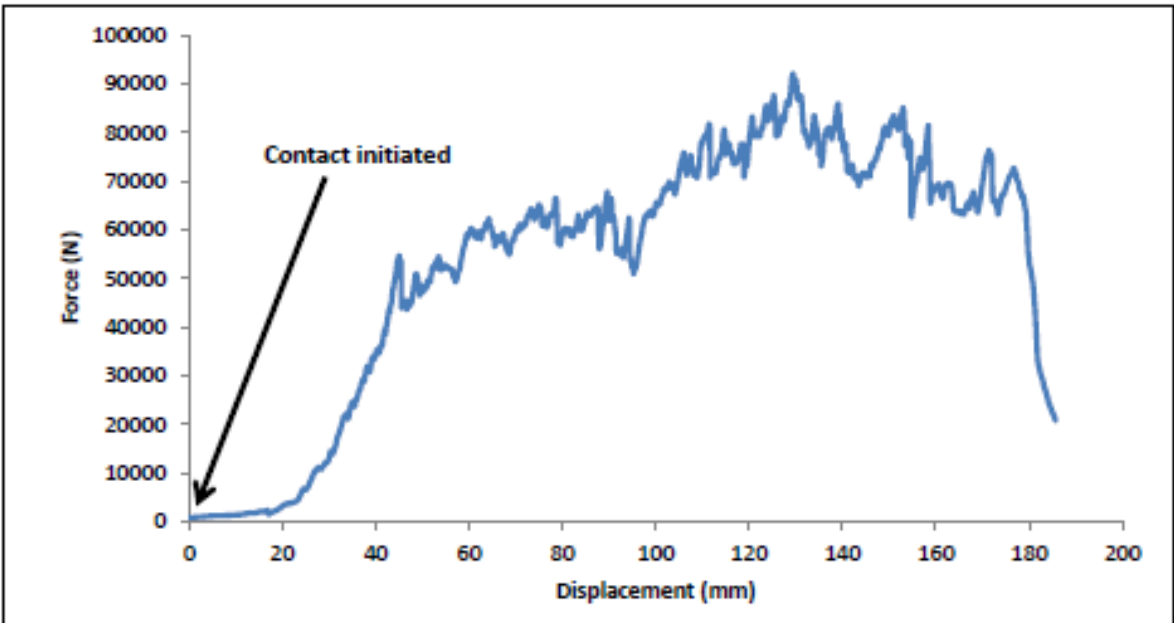

Figure 1: Force-Displacement Curve (dynamic tests nust show displacement during collision and after the point $v=0$ and unil force becomes $=0$ )

\section{ATTACH PROOF OF EQUTVAIENCY} TECHNICAL COMMITIEE DECTSION/COMMENTS

Approved by Date

NOTE: THIS FORM AND THE APPROVED COPY OF THE SUBMISSION MUST BE PRESENTED AT TECHNICAL INSPECTION AT EVERY FORMULA SAE EVENT ENTERED 
APPENDIX B-2

2013 FSAE $^{\mathrm{TM}}$ IMIPACT ATTENUATOR DATA REPORT - Page 2 of 5

University Name: California Polytechnic State Univ - SLO Car Number(s) \& Event(s): 047 FS.AE Lincoln

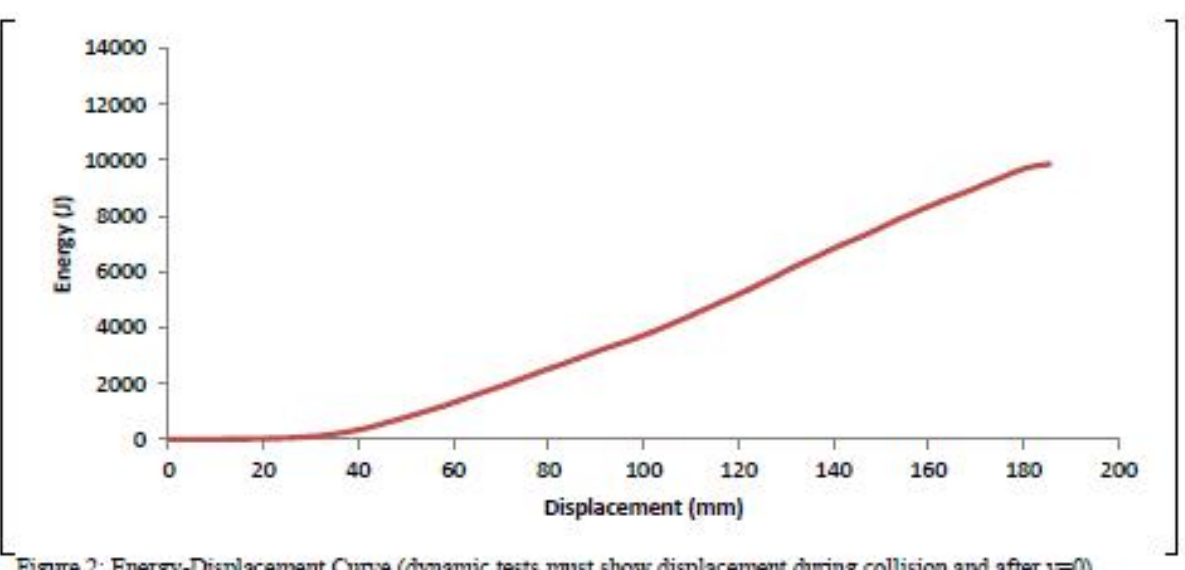

Figure 2: Energy-Displacement Curve (dynamic tests nust show displacement during collision and after $\mathrm{v}=0$ )

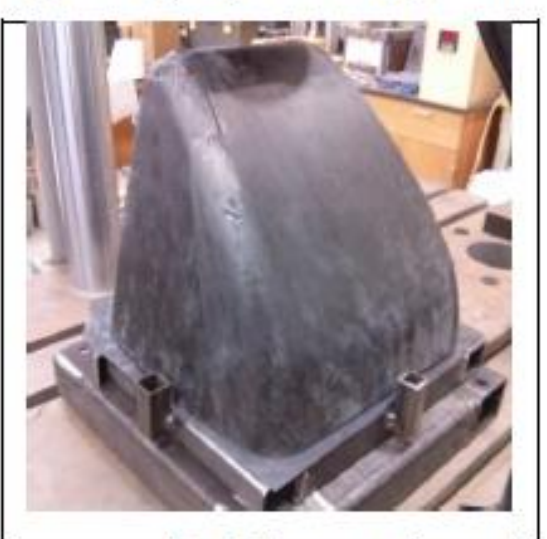

Figure 3: Attenuator as Constructed

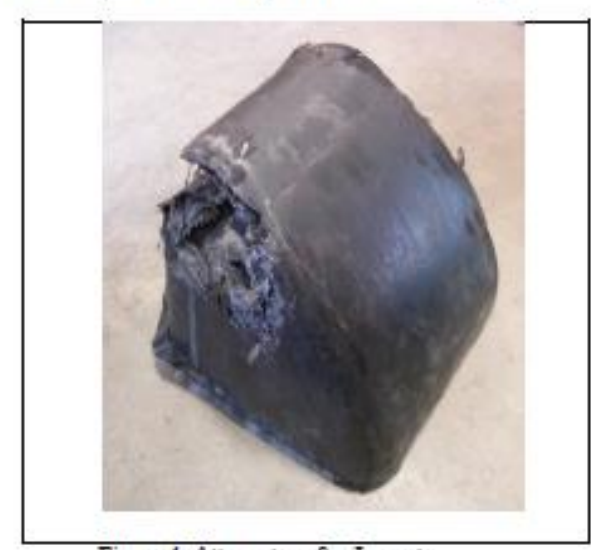

Figure 4: Attenuator affer Inpact (see attachment for AI plate)

\begin{tabular}{|l|l|l|l|}
\hline $\begin{array}{l}\text { Energy Absorbed (J): } \\
\text { Must be }=7350 \mathrm{~J}\end{array}$ & $7350 \mathrm{~J}$ & $\begin{array}{l}\text { Vehicle includes frout wing } \\
\text { in fromt of front bullkhead? }\end{array}$ & No \\
\hline $\begin{array}{l}\text { IA Crusbed Displacement } \\
\text { (mm): }\end{array}$ & $147 \mathrm{~mm}$ & $\begin{array}{l}\text { Wing structure included in } \\
\text { test? }\end{array}$ & No \\
\hline $\begin{array}{l}\text { IA Post Crush Displacement - } \\
\text { demonstrating any renum (nm): }\end{array}$ & $130 \mathrm{~mm}$ & $\begin{array}{l}\text { Test Type: (e.g. barnier test, } \\
\text { drop test, quasi-static crush) }\end{array}$ & Quasi-static crush \\
\hline $\begin{array}{l}\text { Amt-Intrusion Plate } \\
\text { Deformation (mm) }\end{array}$ & $0 \mathrm{mum}$ & $\begin{array}{l}\text { Test Site: (nulst be from } \\
\text { approved test site list on } \\
\text { website for dynamic tests) }\end{array}$ & $\begin{array}{l}\text { Cal Poly Civil } \\
\text { Engiveering Dept. }\end{array}$ \\
\hline
\end{tabular}


APPENDIX B-2

2013 FSAE $^{\mathrm{TM}}$ IMPACT ATTENUATOR DATA REPORT - Page 3 of 5

University Name: California Polytechnic State Univ - SLO Car Number(s) \& Event(s): 047 FS.AE Lincoln
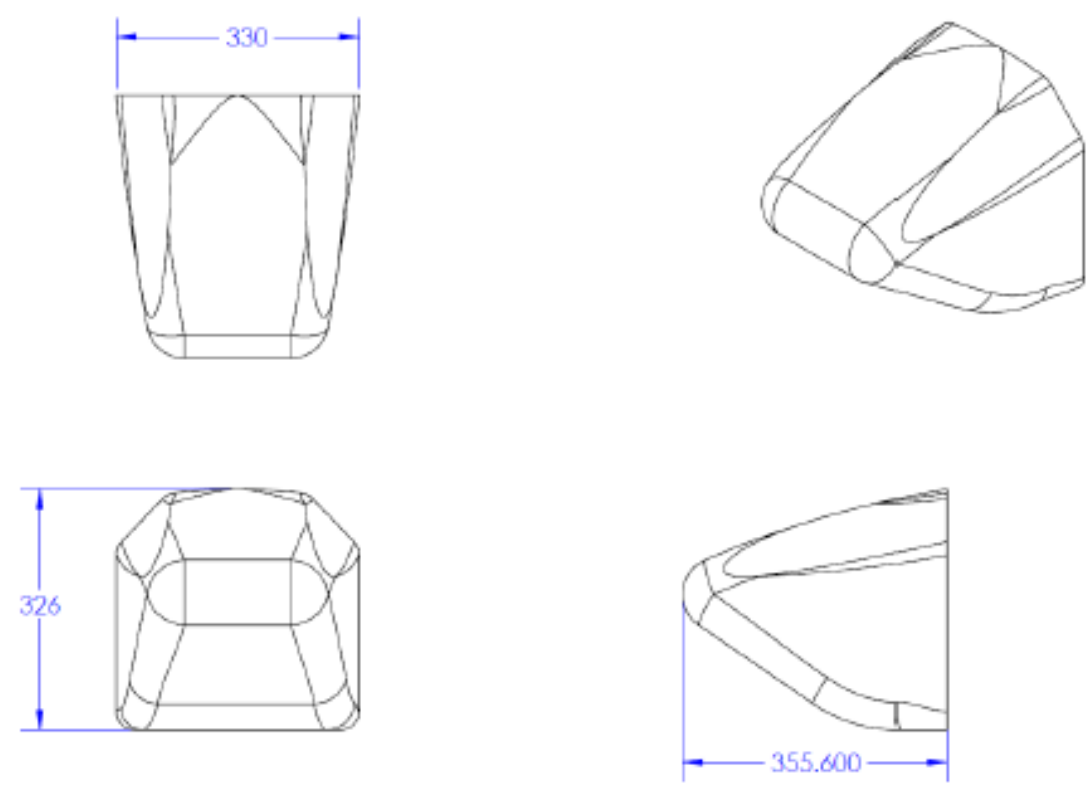

Figure 5: Design Drawings

Length (fore/aft direction): $355 \quad \mathrm{~mm}(=200 \mathrm{~mm}$ )

Width (lateral direction): $330 \mathrm{~mm}(=200 \mathrm{~mm})$

Height (vertical direction): $326 \mathrm{~mm}$ ( $=100 \mathrm{~mm}$ )

Atternator is at least $200 \mathrm{~mm}$ wide by $100 \mathrm{~mm}$ high for at least $200 \mathrm{~mm}$ : Yes

Attach additional information below this point and/or on addirional sheets

Test schematic, photos of test, design report including reasons for selection and advantages/disadvantages, etc. Additional information shall be kept concise and relevant. 
APPENDIX B-2

2013 FSAE $^{\mathrm{TM}}$ IMPACT ATTENUATOR DATA REPORT - Page 4 of 5

University Name: California Polytechnic State Univ - SLO Car Number(s) \& Event(s): 047 FS.AE Lincoln

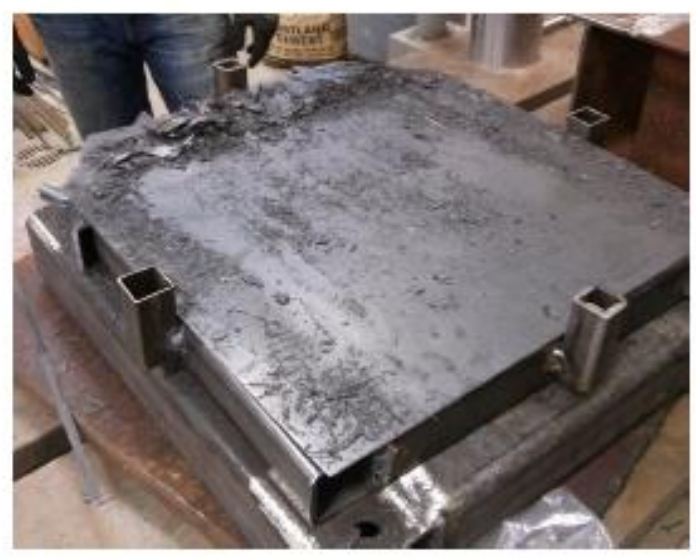

Figure 6: Anti-Intrusion Plate after test.

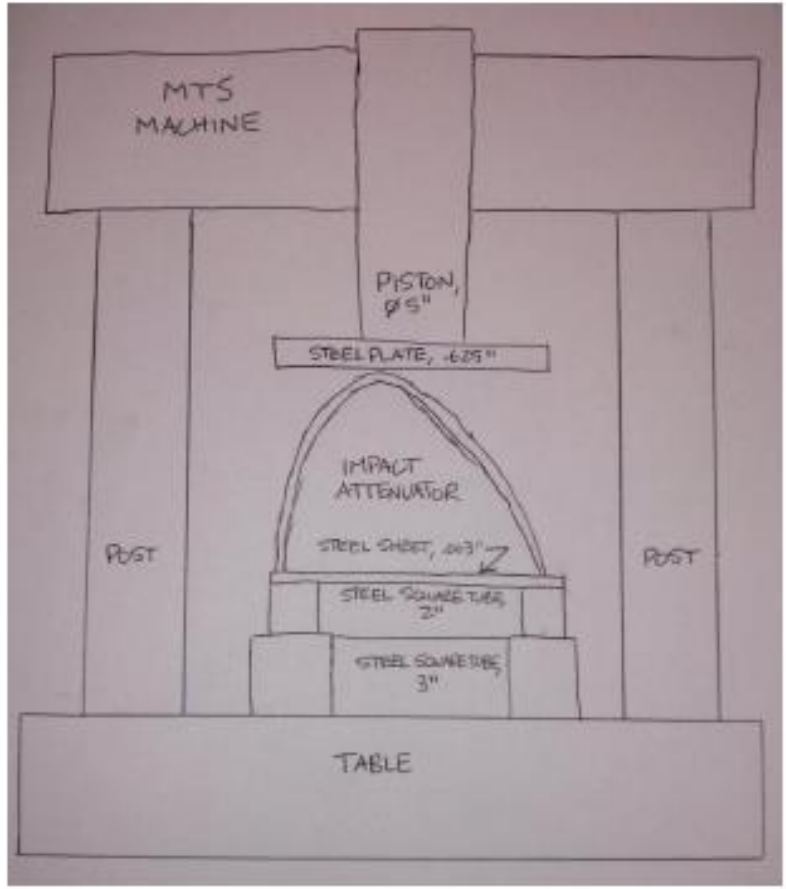

Figure 7: Test semp. 
APPENDIX B-?

2013 FSAETM IMPACT ATTENUATOR DATA REPORT - Page 5 of 5

University Name: California Polvtechnic State Univ - SLO Car Number(s) \& Event(s): 047 FS.AE Lincoln

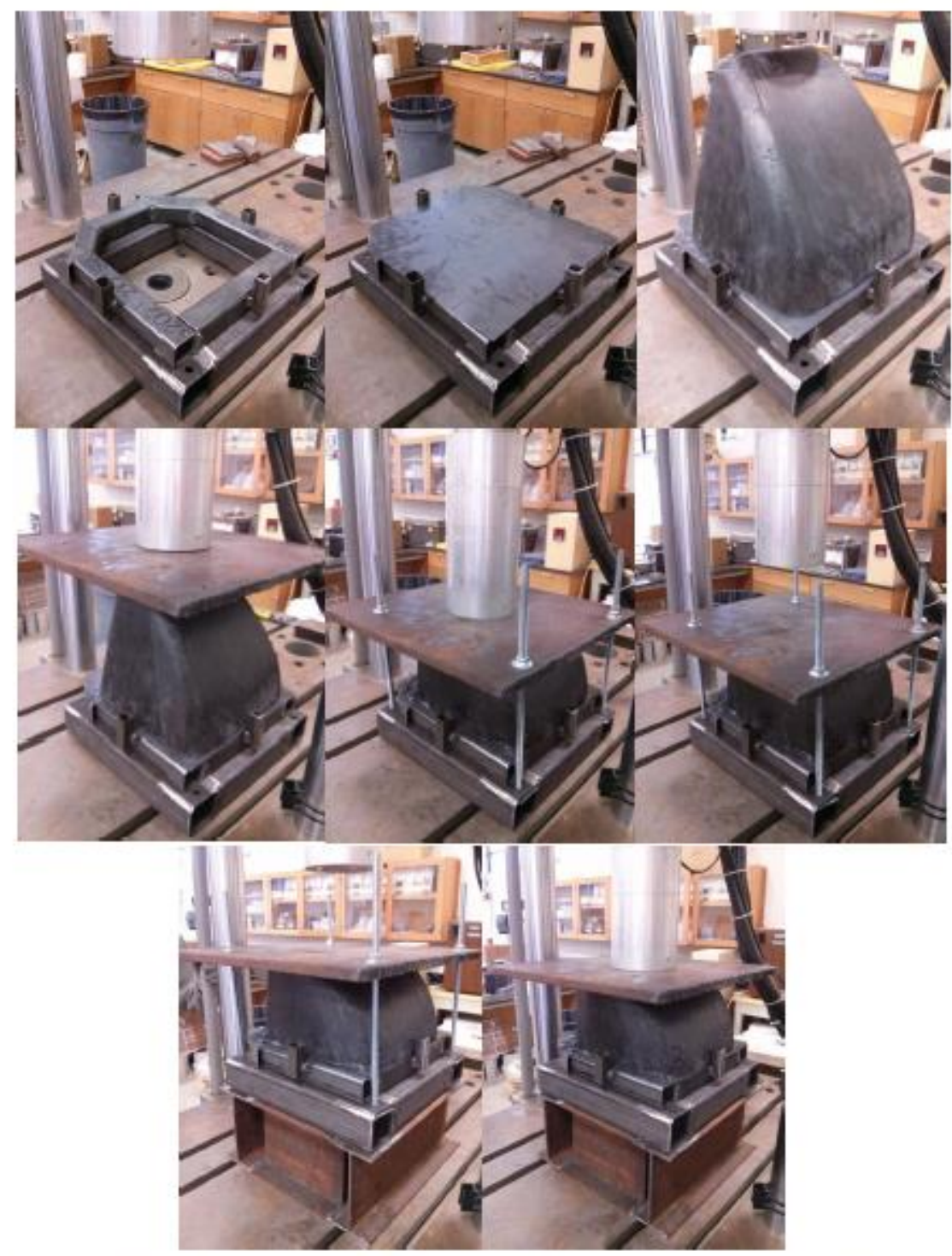

Figure 8: Test procedure, from setup to end of test. A mid-test change to account for lack of piston travel is shoun This change occurs at $145 \mathrm{~mm}$ of displacement. 
Latest abbreviated tube crush input file.

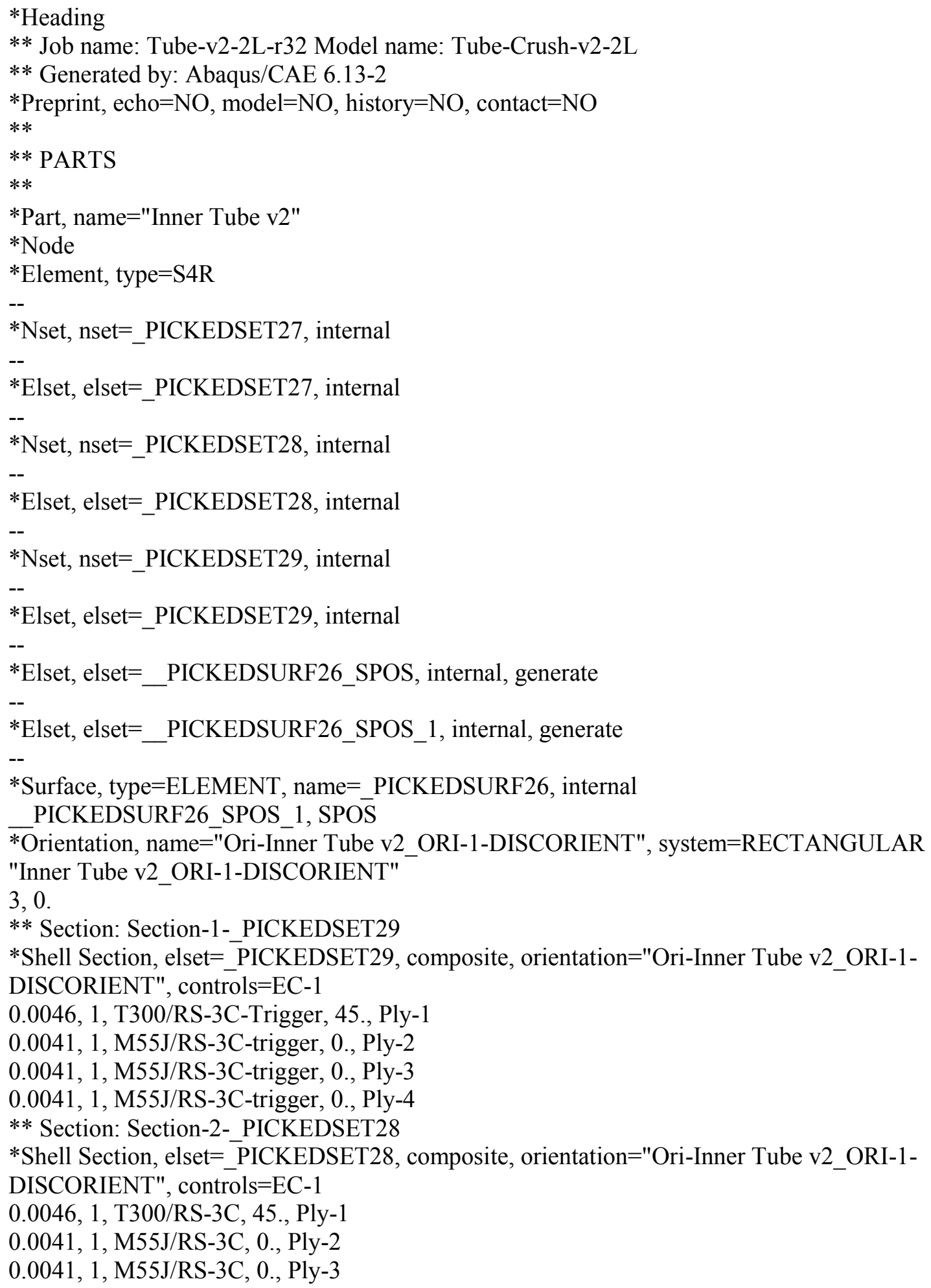


0.0041, 1, M55J/RS-3C, 0., Ply-4

0.0041, 1, M55J/RS-3C, 0., Ply-5

$0.0041,1$, M55J/RS-3C, 0., Ply-6

$0.0041,1$, M55J/RS-3C, 0., Ply-7

0.0041, 1, M55J/RS-3C, 0., Ply-8

*Distribution, name="Inner Tube v2_ORI-1-DISCORIENT", location=ELEMENT, Table="Inner Tube v2_ORI-1-DISCORIENT_Table"

$--$

*End Part

**

*Part, name="Outter Tube v2"

*Node

$--$

*Element, type $=\mathrm{S} 4$

$-$

*Element, type $=\mathrm{S} 4 \mathrm{R}$

$-$

*Nset, nset=_PICKEDSET19, internal

$--$

*Elset, elset=_PICKEDSET19, internal

$--$

*Nset, nset $=$ _PICKEDSET20, internal

$--$

*Elset, elset=_PICKEDSET20, internal

$--$

*Nset, nset=_PICKEDSET21, internal

$--$

*Elset, elset=_PICKEDSET21, internal

$--$

*Elset, elset $=$ _PICKEDSURF18_SPOS, internal, generate

$--$

*Elset, elset $=$ _PICKEDSURF18_SPOS_1, internal, generate

$--$

*Surface, type=ELEMENT, name=_PICKEDSURF18, internal PICKEDSURF18_SPOS_1, SPOS

*Orientation, name="Ori-Outter Tube v2_ORI-1-DISCORIENT-2", system=RECTANGULAR "Outter Tube v2_ORI-1-DISCORIENT-2"

3,0 .

** Section: Section-3-_PICKEDSET21

*Shell Section, elset=_PICKEDSET21, composite, orientation="Ori-Outter Tube v2_ORI-1DISCORIENT-2", controls=EC-1

0.0041, 1, M55J/RS-3C-trigger, 0., Ply-1

0.0041, 1, M55J/RS-3C-trigger, 0., Ply-2

0.0041, 1, M55J/RS-3C-trigger, 0., Ply-3

0.0041, 1, M55J/RS-3C-trigger, 0., Ply-4

** Section: Section-4-_PICKEDSET20

*Shell Section, elset=_PICKEDSET20, composite, orientation="Ori-Outter Tube v2_ORI-1DISCORIENT-2", controls=EC-1

0.0041, 1, M55J/RS-3C, 0., Ply-1

$0.0041,1$, M55J/RS-3C, 0., Ply-2

0.0041, 1, M55J/RS-3C, 0., Ply-3 
0.0041, 1, M55J/RS-3C, 0., Ply-4

0.0041, 1, M55J/RS-3C, 0., Ply-5

0.0041, 1, M55J/RS-3C, 0., Ply-6

$0.0041,1$, M55J/RS-3C, 0., Ply-7

$0.0046,1, \mathrm{~T} 300 / \mathrm{RS}-3 \mathrm{C}, 45$., Ply-8

*Distribution, name="Outter Tube v2_ORI-1-DISCORIENT-2", location=ELEMENT, Table="Outter Tube v2_ORI-1-DISCORIENT-2_Table"

$--$

*End Part

**

*Part, name=PLATE-1

*End Part

$* *$

$* *$

** ASSEMBLY

$* *$

*Assembly, name $=$ Assembly

**

*Instance, name=PLATE-1, part=PLATE-1

$0 ., \quad 0 ., \quad 3.001$

*Node

$\begin{array}{llllll}0 ., & 0 ., & 3.001, & 1 ., & 0 ., & 3.001,\end{array}$

$1, \quad 0 ., \quad 0 ., \quad 0$.

*Nset, nset=PLATE-1-RefPt_, internal

1 ,

*Nset, nset=PLATE-1-REFPT , internal

1 ,

*Surface, type=CYLINDER, name="PLATE-1-Plate Surface"

START, $5 ., \quad 0$.

LINE, $\quad-5 ., \quad 0$.

*End Instance

**

*Instance, name="Inner Tube v2-1", part="Inner Tube v2"

*End Instance

**

*Instance, name="Outter Tube v2-1", part="Outter Tube v2"

*End Instance

**

*Nset, nset=PLATERP, instance=PLATE-1

1 ,

*Nset, nset="Tube Base", instance="Inner Tube v2-1"

$-$

*Nset, nset="Tube Base", instance="Outter Tube v2-1"

$--$

*Elset, elset="Tube Base", instance="Inner Tube v2-1"

*Elset, elset="Tube Base", instance="Outter Tube v2-1"

$--$

*Elset, elset="_Inner Tube Surf_", internal, instance="Inner Tube v2-1", generate -

*Elset, elset="_Outter Tube Surf_", internal, instance="Outter Tube v2-1" 
$--$

*Surface, type=ELEMENT, name="Outter Tube Surf"

"_Outter Tube Surf ",

** Constraint: Rigi $\overline{d B o d y-1}$

*Rigid Body, ref node=PLATE-1.PLATE-1-REFPT_, analytical surface=PLATE-1."PLATE-1-

Plate Surface"

*End Assembly

$* *$

** ELEMENT CONTROLS

$* *$

*Section Controls, name=EC-1, ELEMENT DELETION=YES, MAX DEGRADATION=0.99, hourglass $=$ RELAX STIFFNESS

1., $1 ., 1$.

*Amplitude, name $=$ SMOOTHSTEP, definition=SMOOTH STEP
0 ,
0 ,
0.15 ,
1.

** MATERIALS

$* *$

$* *$ Density $=[1 \mathrm{bf} * \mathrm{~s} * * 2 / \mathrm{in} * * 4]$

$* *$ Moduli $=[\mathrm{lbf} / \mathrm{in} * * 2]$

$* *$ Strengths $=\left[\mathrm{lbf} / \mathrm{in}^{* * 2}\right]$

** Fracture Energy $=[\mathrm{lbf} / \mathrm{in}]$

*Material, name $=$ M55J/RS-3C

*Damage Initiation, criterion=HASHIN

---> OMITTED

* Damage Evolution, type $=$ ENERGY

2.26411e+06, 639238., 16110.8, 270586.

*Density

0.0001501 ,

*Elastic, type=LAMINA

---> OMITTED

$* *$ Density $=\left[1 \mathrm{bf} * \mathrm{~s} * * 2 / \mathrm{in}^{* * 4} 4\right]$

$* *$ Moduli $=\left[\mathrm{lbf} / \mathrm{in}^{* * 2} 2\right]$

$* *$ Strengths $=[\mathrm{lbf} / \mathrm{in} * * 2]$

$* *$ Fracture Energy $=[1 \mathrm{bf} / \mathrm{in}]$

*Material, name $=$ M55J/RS-3C-trigger

*Damage Initiation, criterion=HASHIN

---> OMITTED

*Damage Evolution, type=ENERGY

2.26411e+06, 639238., 16110.8, 270586.

*Density

0.0001501 ,

*Elastic, type=LAMINA

---> OMITTED

** See notes for M55J/RS-3C

*Material, name $=\mathrm{T} 300 / \mathrm{RS}-3 \mathrm{C}$

*Damage Initiation, criterion=HASHIN

---> OMITTED

*Damage Evolution, type=ENERGY

2.26411e+06， 639238., 2.26411e+06， 639238.

*Density 
0.0001449 ,

*Elastic, type=LAMINA

---> OMITTED

** See notes for M55J/RS-3C

*Material, name $=\mathrm{T} 300 / \mathrm{RS}-3 \mathrm{C}$-Trigger

*Damage Initiation, criterion=HASHIN

---> OMITTED

*Damage Evolution, type $=$ ENERGY

2.26411e+06, 639238., 2.26411e+06, 639238.

*Density

0.0001449 ,

*Elastic, type=LAMINA

---> OMITTED

**

** INTERACTION PROPERTIES

$* *$

*Surface Interaction, name $=$ DEFAULT

*Surface Interaction, name $=$ IMPACTCONTACT

*Friction

0.2 ,

*Surface Behavior, pressure-overclosure $=$ HARD

*Contact Damping, definition=DAMPING COEFFICIENT

0.2 ,

*Surface Interaction, name=NOFRICT

*Friction

0 .,

*Surface Behavior, pressure-overclosure $=$ HARD

*Distribution Table, name="Inner Tube v2_ORI-1-DISCORIENT_Table" coord3d, coord3d

*Distribution Table, name="Outter Tube v2_ORI-1-DISCORIENT-2_Table"

coord3d, coord3d

**

** BOUNDARY CONDITIONS

$* *$

** Name: Disp-BC-1 Type: Displacement/Rotation

*Boundary

"Tube Base", 1, 1

** Name: Disp-BC-2 Type: Displacement/Rotation

*Boundary

"Tube Base", 2, 2

** Name: Disp-BC-3 Type: Displacement/Rotation

*Boundary

"Tube Base", 3, 3

** Name: Disp-BC-4 Type: Displacement/Rotation

*Boundary

"Tube Base", 4, 4

** Name: Disp-BC-5 Type: Displacement/Rotation

*Boundary

"Tube Base", 5, 5

** Name: Disp-BC-6 Type: Displacement/Rotation

*Boundary 
PLATERP, 1,1

** Name: Disp-BC-7 Type: Displacement/Rotation

*Boundary

PLATERP, 2, 2

** Name: Disp-BC-8 Type: Displacement/Rotation

*Boundary

PLATERP, 4, 4

** Name: Disp-BC-9 Type: Displacement/Rotation

*Boundary

PLATERP, 5, 5

** Name: Disp-BC-10 Type: Displacement/Rotation

*Boundary

PLATERP, 6, 6

$* *$

$* *$

** STEP: Crush

$* *$

*Step, name $=$ Crush, nlgeom $=$ YES

*Dynamic, Explicit

, 0.5

*Bulk Viscosity

$0.06,1.2$

** Mass Scaling: Semi-Automatic

** Whole Model

$*$ Variable Mass Scaling, $\mathrm{dt}=5 \mathrm{e}-06$, type $=$ below min, frequency $=250$

$* *$

** BOUNDARY CONDITIONS

$* *$

** Name: Vel-BC-1 Type: Velocity/Angular velocity

*Boundary, amplitude=SMOOTHSTEP, type=VELOCITY

PLATERP, 3, 3, -1.

$* *$

** INTERACTIONS

$* *$

** Interaction: general_contact

*Contact, op=NEW

*Contact Inclusions, ALL EXTERIOR

*Contact Property Assignment

, , NOFRICT

PLATE-1."PLATE-1-Plate Surface" , "Inner Tube Surf", IMPACTCONTACT

PLATE-1."PLATE-1-Plate Surface" , "Outter Tube Surf" , IMPACTCONTACT

*Surface Property Assignment, property=THICKNESS

"Inner Tube Surf", ORIGINAL , 1.

"Outter Tube Surf", ORIGINAL , 1.

**

** OUTPUT REQUESTS

$* *$

$*$ Restart, write, number interval $=1$, time marks $=\mathrm{NO}$

$* *$

** FIELD OUTPUT: F-Output-1

$* *$ 
*Output, field

*Node Output

A, RF, U, V

$* *$

** FIELD OUTPUT: F-Output-3

$* *$

*Contact Output CSTRESS,

**

** FIELD OUTPUT: F-Output-2

$* *$

*Element Output, directions $=$ YES

DAMAGEC, DAMAGEFC, DAMAGEFT, DAMAGET, EVF, LE, PE, PEEQ, PEEQVAVG, PEVAVG, S, STATUS, SVAVG

**

** HISTORY OUTPUT: H-Output-2

$* *$

*Output, history, filter=ANTIALIASING

*Node Output, nset=PLATERP

RF3, RM1, RM2, RM3, U3

$* *$

** HISTORY OUTPUT: H-Output-1

$* *$

*Output, history, variable=PRESELECT

*End Step 
Abbreviated version of the nosecone crush input file.

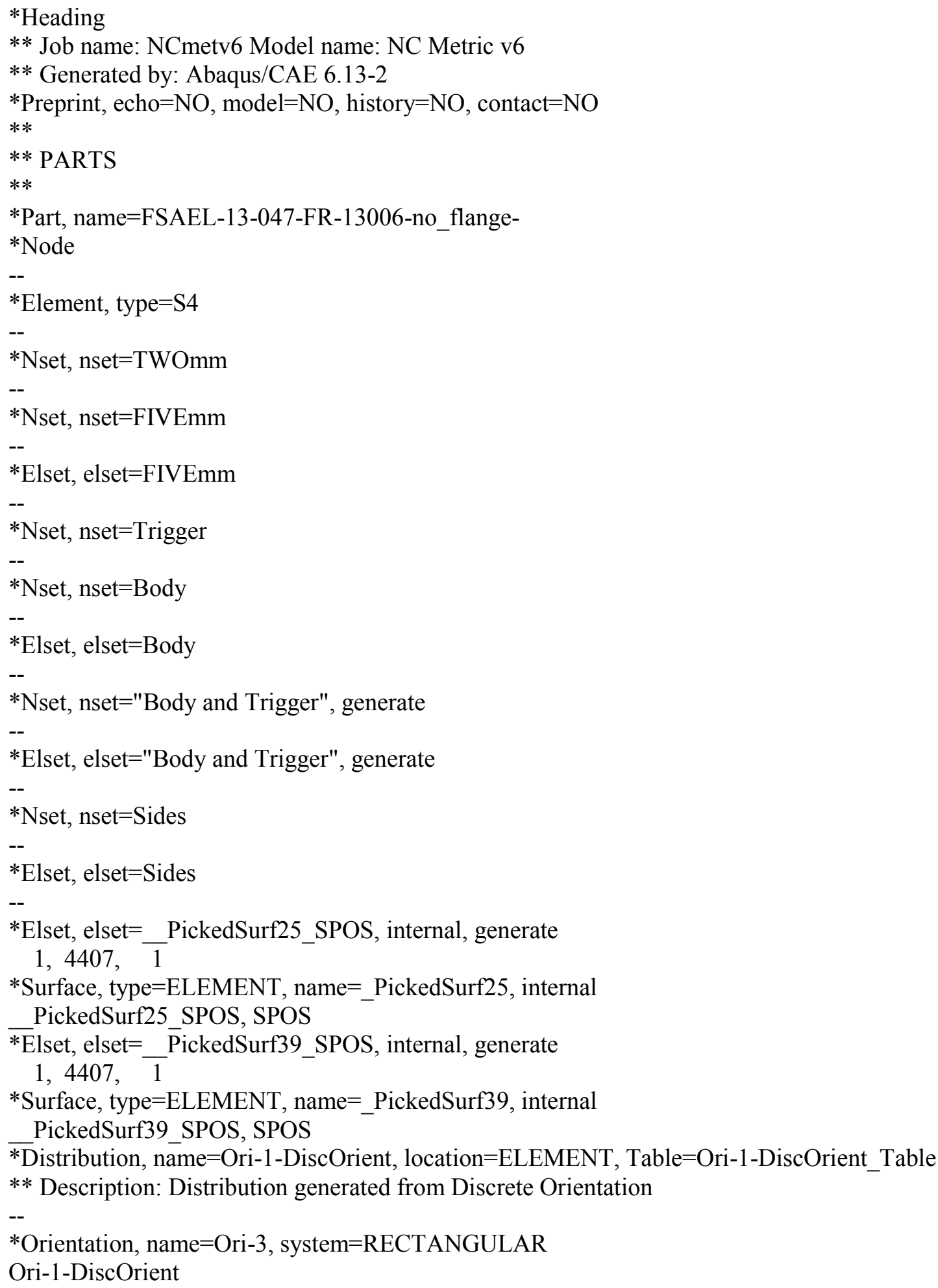


3,0 .

** Region: (CompositeLayup-1-3: Generated From Layup), (Controls:EC-1)

*Elset, elset=CompositeLayup-1-3

$--$

** Section: CompositeLayup-1-3

*Shell Section, elset=CompositeLayup-1-3, composite, orientation=Ori-3, controls=EC-1, layup=CompositeLayup-1

$0.15,1, \mathrm{~T} 700 / \mathrm{E} 765,0 ., \mathrm{B}-1$

$0.15,1, \mathrm{~T} 700 / \mathrm{E} 765,90 ., \mathrm{B}-2$

$0.15,1, \mathrm{~T} 700 / \mathrm{E} 765,0 ., \mathrm{B}-3$

$0.15,1, \mathrm{~T} 700 / \mathrm{E} 765,90 ., \mathrm{BT}-4$

$0.15,1, \mathrm{~T} 700 / \mathrm{E} 765,0 ., \mathrm{BT}-5$

$0.15,1, \mathrm{~T} 700 / \mathrm{E} 765,90 ., \mathrm{BT}-6$

$0.15,1, \mathrm{~T} 700 / \mathrm{E} 765,0 ., \mathrm{B}-7$

0.15, 1, T700/E765, 90., B-8

$0.15,1, \mathrm{~T} 700 / \mathrm{E} 765,0 ., \mathrm{B}-9$

*Orientation, name $=$ Ori-2, system $=$ RECTANGULAR

Ori-1-DiscOrient

3,0 .

** Region: (CompositeLayup-1-2: Generated From Layup), (Controls:EC-1)

*Elset, elset=CompositeLayup-1-2

$--$

** Section: CompositeLayup-1-2

*Shell Section, elset=CompositeLayup-1-2, composite, orientation=Ori-2, controls=EC-1, layup=CompositeLayup-1

$0.15,1, \mathrm{~T} 700 / \mathrm{E} 765,0 ., \mathrm{B}-1$

$0.15,1, \mathrm{~T} 700 / \mathrm{E} 765,90 ., \mathrm{B}-2$

$0.15,1, \mathrm{~T} 700 / \mathrm{E} 765,0 ., \mathrm{B}-3$

0.15, 1, T700/E765, 90., BT-4

$0.15,1, \mathrm{~T} 700 / \mathrm{E} 765,0 ., \mathrm{BT}-5$

$0.15,1, \mathrm{~T} 700 / \mathrm{E} 765,90$., BT-6

$0.15,1, \mathrm{~T} 700 / \mathrm{E} 765,0 ., \mathrm{B}-7$

$0.15,1, \mathrm{~T} 700 / \mathrm{E} 765,90 ., \mathrm{B}-8$

0.15, 1, T700/E765, 0., B-9

$0.15,1, \mathrm{~T} 700 / \mathrm{E} 765,90 ., \mathrm{S}-10$

$0.15,1, \mathrm{~T} 700 / \mathrm{E} 765,0 ., \mathrm{S}-11$

$0.15,1, \mathrm{~T} 700 / \mathrm{E} 765,90 ., \mathrm{S}-12$

$0.15,1, \mathrm{~T} 700 / \mathrm{E} 765,0 ., \mathrm{S}-13$

0.15, 1, T700/E765, 90., S-14

$0.15,1, \mathrm{~T} 700 / \mathrm{E} 765,0 ., \mathrm{S}-15$

0.15, 1, T700/E765, 90., S-16

$0.15,1, \mathrm{~T} 700 / \mathrm{E} 765,0 ., \mathrm{S}-17$

0.15, 1, T700/E765, 90., S-18

*Orientation, name=Ori-1, system $=$ RECTANGULAR

Ori-1-DiscOrient

3, 0 .

** Region: (CompositeLayup-1-1: Generated From Layup), (Controls:EC-2)

*Elset, elset=CompositeLayup-1-1

$--$

** Section: CompositeLayup-1-1 
*Shell Section, elset=CompositeLayup-1-1, composite, orientation=Ori-1, controls=EC-2, layup=CompositeLayup-1

$0.15,1, \mathrm{~T} 700 / \mathrm{E} 765,90 ., \mathrm{BT}-4$

$0.15,1, \mathrm{~T} 700 / \mathrm{E} 765,0 ., \mathrm{BT}-5$

$0.15,1, \mathrm{~T} 700 / \mathrm{E} 765,90 .$, BT-6

*End Part

$* *$

*Part, name=Impactor

*End Part

$* *$

$* *$

$* *$ ASSEMBLY

$* *$

*Assembly, name=Assembly

$* *$

*Instance, name=FSAEL-13-047-FR-13006-no_flange--1, part=FSAEL-13-047-FR-13006no_flange$0 ., \quad-321.35906, \quad-469.9$

*End Instance

$* *$

*Instance, name $=$ Impactor -1 , part $=$ Impactor

0., $\quad 0 ., 342.903762207031$

0., $\quad 0 ., 342.903762207031, \quad 1 ., \quad 0 ., 342.903762207031, \quad 90$.

*Node

1, $\quad 0 ., \quad 0 ., \quad 0$.

*Nset, nset=Impactor-1-RefPt_, internal

1 ,

*Surface, type=CYLINDER, name="Impactor Surface"

START, 250., 0 .

LINE, -250, $\quad 0$.

*Rigid Body, ref node=Impactor-1-RefPt_, analytical surface="Impactor Surface"

*End Instance

**

*Nset, nset=Base-Sides, instance=FSAEL-13-047-FR-13006-no_flange--1

$--$

*Elset, elset=Base-Sides, instance=FSAEL-13-047-FR-13006-no_flange--1

--

*Nset, nset=Base-TopBot, instance=FSAEL-13-047-FR-13006-no_flange--1

$--$

*Elset, elset=Base-TopBot, instance=FSAEL-13-047-FR-13006-no_flange--1

$--$

*Nset, nset=NCbaseALL, instance=FSAEL-13-047-FR-13006-no_flange--1

$--$

*Elset, elset=NCbaseALL, instance=FSAEL-13-047-FR-13006-no_flange--1

$--$

*Nset, nset=NCbasePIN, instance=FSAEL-13-047-FR-13006-no_flange--1

$--$

*Elset, elset=NCbasePIN, instance=FSAEL-13-047-FR-13006-no_flange--1, generate

$--$

*Nset, nset=NCpin, instance=FSAEL-13-047-FR-13006-no_flange--1

$--$ 


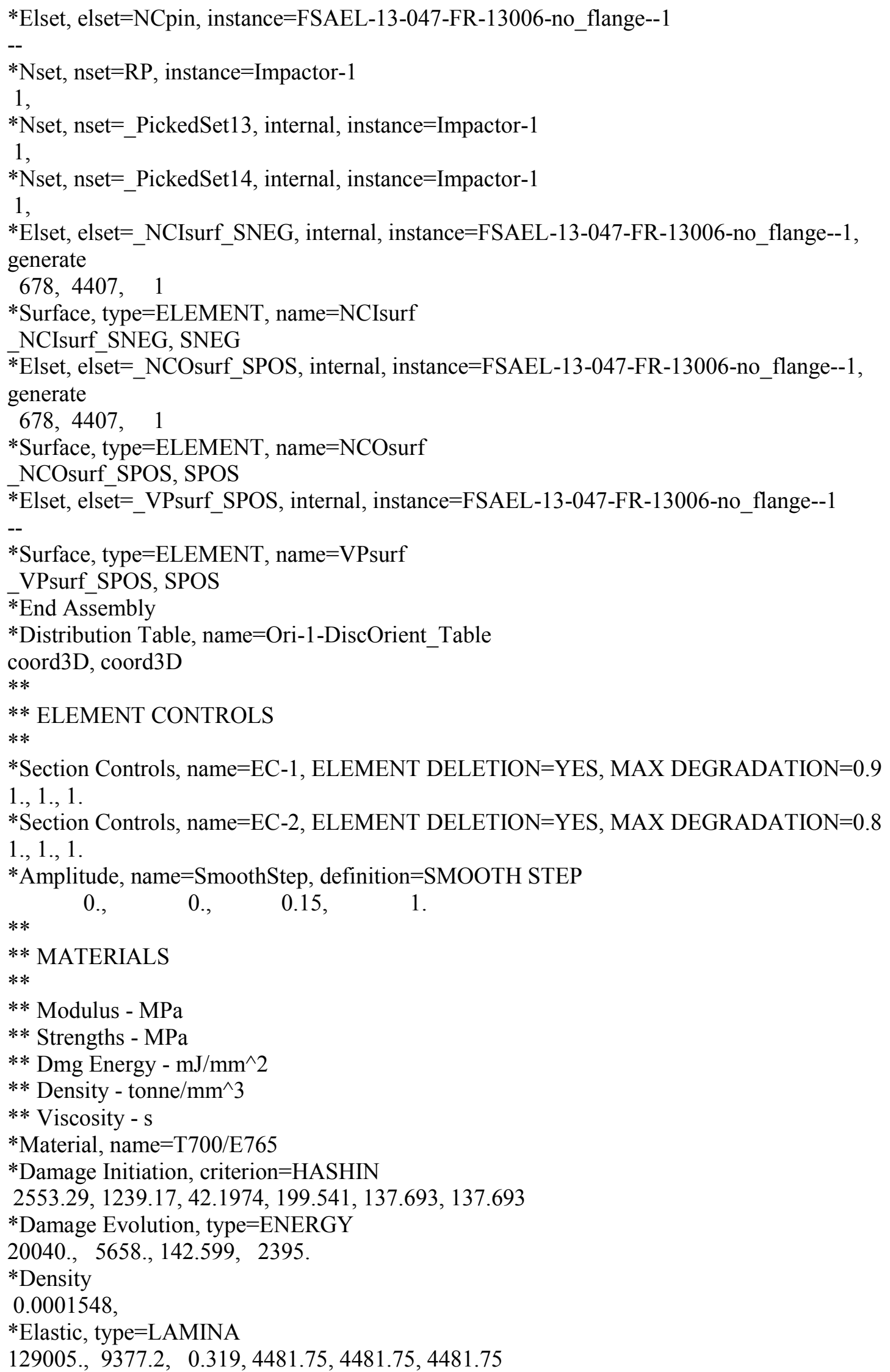




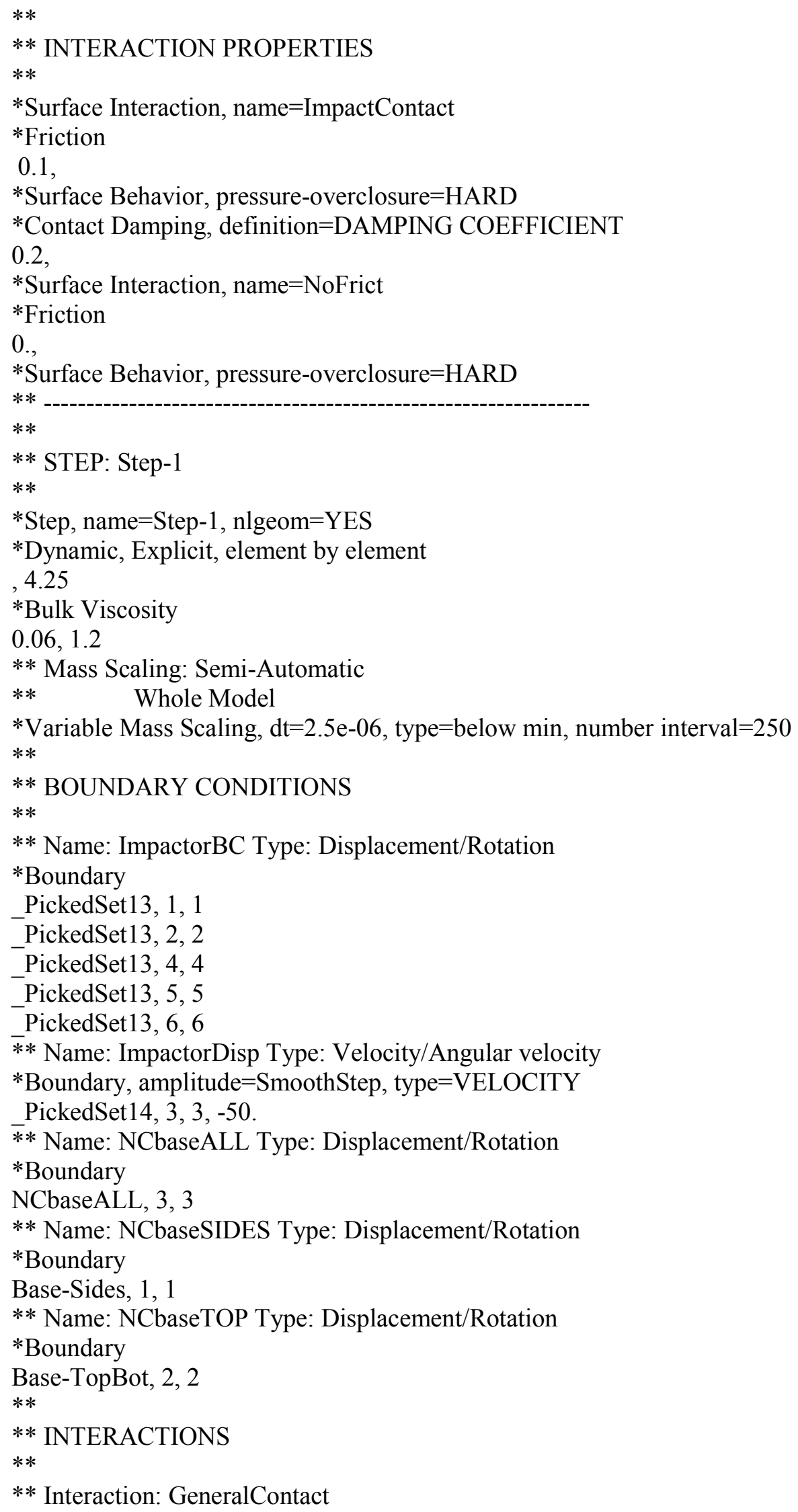




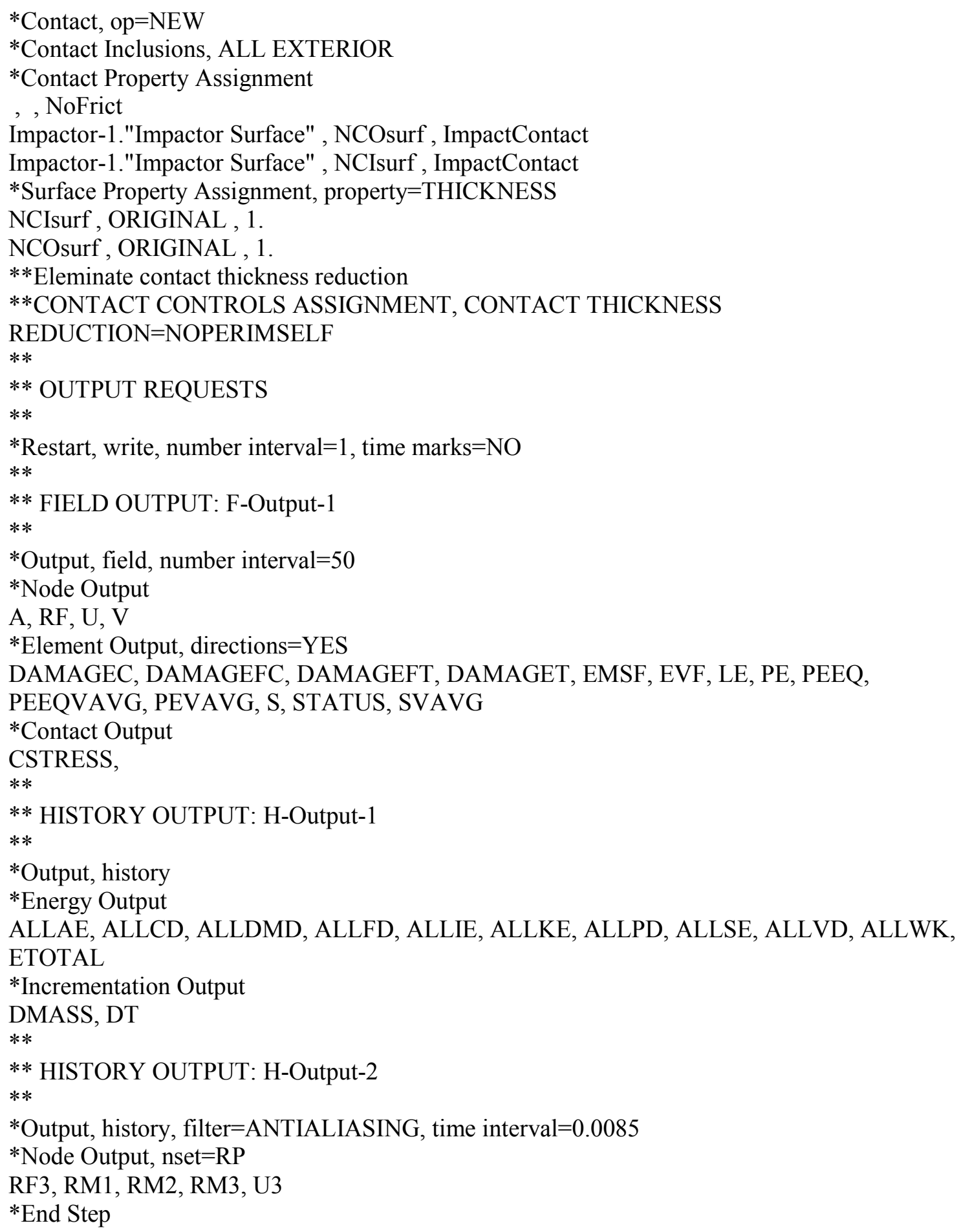

